\title{
ERSEM 15.06: a generic model for marine biogeochemistry and the ecosystem dynamics of the lower trophic levels
}

\author{
Momme Butenschön $^{1}$, James Clark ${ }^{1}$, John N. Aldridge ${ }^{2}$, Julian Icarus Allen ${ }^{1,3}$, Yuri Artioli ${ }^{1}$, Jeremy Blackford ${ }^{1}$, \\ Jorn Bruggeman ${ }^{1}$, Pierre Cazenave ${ }^{1}$, Stefano Ciavatta ${ }^{1,3}$, Susan Kay ${ }^{1}$, Gennadi Lessin $^{1}$, Sonja van Leeuwen ${ }^{2}$, \\ Johan van der Molen ${ }^{2}$, Lee de Mora ${ }^{1}$, Luca Polimene ${ }^{1}$, Sevrine Sailley ${ }^{1}$, Nicholas Stephens ${ }^{1}$, and Ricardo Torres ${ }^{1}$ \\ ${ }^{1}$ Plymouth Marine Laboratory, Prospect Place, The Hoe, Plymouth, PL1 3DH, UK \\ ${ }^{2}$ Centre for Environment, Fisheries, and Aquaculture Science, Lowestoft, UK \\ ${ }^{3}$ National Centre for Earth Observation, Plymouth, UK \\ Correspondence to: Momme Butenschön (momm@pml.ac.uk)
}

Received: 6 July 2015 - Published in Geosci. Model Dev. Discuss.: 26 August 2015

Revised: 14 December 2015 - Accepted: 15 March 2016 - Published: 5 April 2016

\begin{abstract}
The European Regional Seas Ecosystem Model (ERSEM) is one of the most established ecosystem models for the lower trophic levels of the marine food web in the scientific literature. Since its original development in the early nineties it has evolved significantly from a coastal ecosystem model for the North Sea to a generic tool for ecosystem simulations from shelf seas to the global ocean. The current model release contains all essential elements for the pelagic and benthic parts of the marine ecosystem, including the microbial food web, the carbonate system, and calcification. Its distribution is accompanied by a testing framework enabling the analysis of individual parts of the model. Here we provide a detailed mathematical description of all ERSEM components along with case studies of mesocosm-type simulations, water column implementations, and a brief example of a full-scale application for the north-western European shelf. Validation against in situ data demonstrates the capability of the model to represent the marine ecosystem in contrasting environments.
\end{abstract}

\section{Introduction}

Over the last 2 decades a number of marine ecosystem models describing ocean biogeochemistry and the lower trophic levels of the food web have emerged in a variety of contexts ranging from simulations of batch cultures or mesocosms over estuarine and coastal systems to the global ocean (e.g. Fasham et al., 1990; Flynn, 2010; Geider et al., 1997;
Wild-Allen et al., 2010; Zavatarelli and Pinardi, 2003; Aumont et al., 2003; Follows et al., 2007; Yool et al., 2013; Stock et al., 2014). Some of them have matured with the years into sound scientific tools in operational forecasting systems and are used to inform policy and management decisions regarding essential issues of modern human society, such as climate change, ecosystem health, food provision, and other ecosystem goods and services (e.g. Lenhart et al., 2010; Glibert et al., 2014; van der Molen et al., 2014; Doney et al., 2012; Bopp et al., 2013; Chust et al., 2014; Barange et al., 2014). Given the importance of these applications, transparent descriptions of the scientific contents of these models are necessary in order to allow full knowledge and assessment of their strength and weaknesses, as well as maintenance and updating according to scientific insight and progress.

Here we provide a full description of one of these models, the European Regional Seas Ecosystem Model (ERSEM), developed in the early nineties (Baretta et al., 1995; Baretta, 1997) ${ }^{1}$ out of a European collaborative effort, building on previous developments (Radford and Joint, 1980; Baretta et al., 1988). Subsequent development of the model has occurred in separate streams, leading to individual versions of the model, the main ones being the ERSEM version de-

\footnotetext{
${ }^{1}$ The two given references are the introductions to two special issues published on the original model versions ERSEM I and II, representing the entire volumes. More specific references to single papers within these volumes are given in the relevant process descriptions.
} 
scribed in Allen et al. (2001), Blackford and Burkill (2002), and Blackford et al. (2004), and the version of Vichi et al. (2004, 2007), Leeuwen et al. (2012), and van der Molen et al. (2014), http://www.nioz.nl/northsea_model, also referred to as the Biogeochemical Flux Model. The present release is based on the former development stream (Blackford et al., 2004). It has since the beginnings of ERSEM gradually evolved into what is now the principal model for shelf-sea applications within the UK and beyond. It is part of the operational suite of the UK Met Office and the biogeochemical component for the north-western European shelf seas within the European Copernicus Marine Service.

While it was originally created as a scientific tool for the North Sea ecosystem (hence the name), it has since evolved considerably in its scientific content, broadening the scope of the model to coastal systems across the globe as well as the open ocean. Allen et al. (2001) adopted the model for simulations across the entire north-western European shelf sea, further extended in Holt et al. (2012) and Artioli et al. (2012) to include the north-eastern Atlantic. Blackford et al. (2004) applied the model across six different ecosystem types across the globe, Barange et al. (2014) used applications of the model in the major coastal upwelling zones of the planet, and Kwiatkowski et al. (2014) assessed the skill of the model, demonstrating its competitiveness with respect to other established global ocean models. The model has been subject to validation on various levels ranging from basic statistical metrics of point-to-point matches to observational data (Shutler et al., 2011; de Mora et al., 2013) to multi-variate analysis (Allen et al., 2007; Allen and Somerfield, 2009) and pattern recognition (Saux Picart et al., 2012).

The model has been applied in a wide number of contexts that include short-term forecasting (Edwards et al., 2012), ocean acidification (Blackford and Gilbert, 2007), climate change (Holt et al., 2012), coupled climate-acidification projections (Artioli et al., 2014a), process studies (Polimene et al., 2012, 2014), biogeochemical cycling (Wakelin et al., 2012), habitat (Villarino et al., 2015), and end-to-end modelling (Barange et al., 2014). The wide range of applications and uses of the model coupled with developments since earlier manuscripts documenting the model (Baretta-Bekker, 1995; Baretta, 1997; Blackford et al., 2004) make a thorough and integral publication of its scientific ingredients overdue.

Being an evolution of former models within the ERSEM family that emerged in parallel to other, separate development streams of the original model, the core elements of the current model version closely resemble earlier versions even if presented in much more detail compared to previous works. We present a model for ocean biogeochemistry and the planktonic and benthic parts of the marine ecosystem that includes explicitly the cycles of the major chemical elements of the ocean (carbon, nitrogen, phosphorus, silicate, and iron); it includes the microbial food web, a sub-module for the carbonate system, calcification, and a full benthic model.
Our main objective with this paper is to provide a full description of all model components, accompanied by simple case studies with low resource requirements that illustrate the model capabilities and enable the interested reader to implement our model and reproduce the test cases shown. For this purpose we present the examples of a mesocosm-type framework and three vertical water-column implementations of opposing character complemented with basic validation metrics against in situ observations. All material required to replicate these test cases, such as parametrization and input files, are provided in the Supplement. In addition, a brief illustration of a full-scale three-dimensional implementation is given to show the model in a large-scale application.

The next section gives an overview of the model and its philosophy, while the two following sections contain the descriptions of the pelagic and benthic components, describe the air-sea and seabed interfaces, and detail some generic terms that are used throughout the model. The model description is complemented by two sections that present different implementations of the model and illustrate the testing framework. We complete the work with a section on optional choices of model configuration and a section on the technical specifications of the software package, licence, and instructions on where and how to access the model code.

\section{The ERSEM model}

ERSEM has been, since its origins, an ecosystem model for marine biogeochemistry, pelagic plankton, and benthic fauna. Its functional types (Baretta et al., 1995; Vichi et al., 2007) are based on their macroscopic role in the ecosystem rather than species or taxa, and its state variables are the major chemical components of each type (carbon, chlorophyll $a$, nitrogen, phosphate, silicate and, optionally, iron). It is composed of a set of modules that compute the rates of change of its state variables given the environmental conditions of the surrounding water body, physiological processes, and predator-prey interactions. In the simplest case, the environmental drivers can be provided offline, or through a simple zero-dimensional box model. However, for more realistic representations, including the important processes of horizontal and vertical mixing (or advection) and biogeochemical feedback, a direct (or online) coupling to a physical driver, such as a three-dimensional hydrodynamic model, is required.

The organisms in the model are categorized along with the main classes of ecosystem function into primary producers, consumers and bacterial decomposers, particulate and dissolved organic matter (POM, DOM) in the pelagic and consumers, bacterial decomposers, and particulate and dissolved organic matter in the benthos. Most of these classes are further subdivided into sub-types to allow for an enhanced plasticity of the system in adapting the ecosystem response to the environmental conditions in comparison to the classical 
nutrient, phytoplankton, zooplankton and detritus (NPZD)type models. Importantly, ERSEM uses a fully dynamic stoichiometry in essentially all its types (with the exception of mesozooplankton, benthic bacteria, and zoobenthos, which use fixed stoichiometric ratios). The model dynamics of a living functional type are generally based on a standard organism that is affected by the assimilation of carbon and nutrients into organic compounds by uptake, and the generic loss processes of respiration, excretion, release, predation and non-predatory mortality (Fig. 1; see also Vichi et al. (2007) - "2. Towards a generic formalism for pelagic biogeochemistry"). In this framework we refer to excretion as inefficiencies of the uptake processes, while the release terms represent regulatory processes of the current nutritional state. More specifically, uptake, which may occur in inorganic or organic form, is given by the external availability, actual requirement, and uptake capacity of the relevant functional type, leading to stochiometric variations in its chemical components that are balanced by losses according to the internal quota and storage capacity. This stoichiometric flexibility allows for a diverse response in between the functional types in adapting to the environmental conditions compared to fixed quota models (e.g. through varying resistance against low nutrient conditions and luxury storages supporting a more realistic evolution of the community structure). Figure 2 illustrates the pathways of these fluxes within the food web of the model.

ERSEM is not designed to directly model cell physiology. Its equations are a synthesis of physiological processes and their macroscopic consequences for larger water bodies in which the distributions of the plankton biomass and organic and inorganic material can be approximated as smooth continuous fields. This is important to keep in mind in smallscale and high-resolution applications where this basic assumption of the continuum hypothesis may break down, in which case the system of partial differential balance equations no longer holds. As a rule of thumb, in order to guarantee the validity of the equations, the modelled scales should at least be an order of magnitude bigger than the organisms modelled and smaller patches.

Mathematically, the set of prognostic equations describing the dynamics of marine biogeochemical states is generally given by

$$
\begin{aligned}
& \frac{\partial c_{\mathrm{p}}}{\partial t}+\boldsymbol{u} \cdot \frac{\partial c_{\mathrm{p}}}{\partial \boldsymbol{x}}+\stackrel{c}{\mathrm{p}}_{\mathrm{sed}} \frac{\partial c_{\mathrm{p}}}{\partial z}=v \frac{\partial^{2} c_{\mathrm{p}}}{\partial \boldsymbol{x}^{2}}+\left.\frac{\partial c_{\mathrm{p}}}{\partial t}\right|_{\mathrm{bgc}}, \\
& \frac{\partial c_{\mathrm{b}}}{\partial t}=\left.\frac{\partial c_{\mathrm{b}}}{\partial t}\right|_{\mathrm{bgc}},
\end{aligned}
$$

where $c_{\mathrm{p}}$ are the pelagic concentrations (per volume) and $c_{\mathrm{b}}$ the benthic contents (per sediment surface area) of each chemical component of the organic model types or the inorganic model components. $w_{c_{\mathrm{p}}}$ is the velocity of gravitational sinking of particles in the water column. $x$ represents the vector of spatial coordinates of which $z$ is the vertical coordinate, being 0 at the sea surface and increasing downwards.

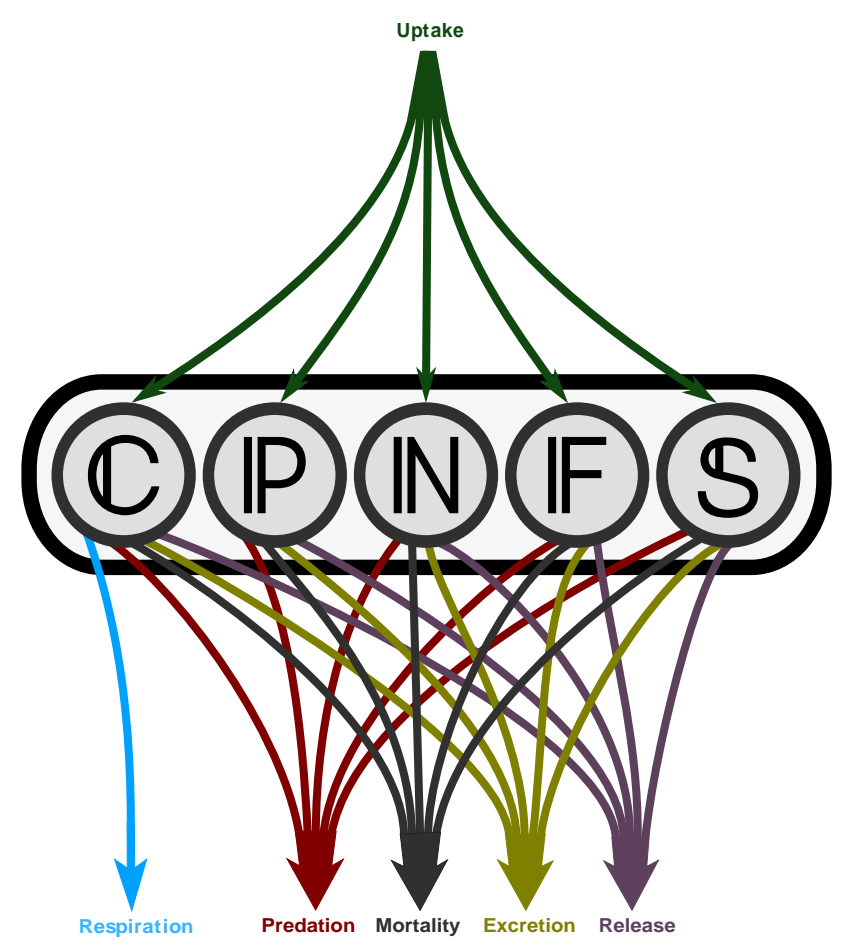

Figure 1. Generic processes acting on the chemical components of the ERSEM standard organism.

The set of equations is closed by the horizontal boundary conditions of the system generally given by the air-sea fluxes $\left.\mathcal{F}\right|_{\text {sea }} ^{\text {air }}$ and the fluxes across the seafloor $\left.\mathcal{F}\right|_{\text {ben }} ^{\text {pel }}$ and lateral boundary conditions if present in the given configuration.

ERSEM computes the biogeochemical rates of change in pelagic $\left(\left.\frac{\partial c_{\mathrm{p}}}{\partial t}\right|_{\mathrm{bgc}}\right)$ and benthic $\left(\left.\frac{\partial c_{\mathrm{b}}}{\partial t}\right|_{\mathrm{bgc}}\right)$ systems, the gas transfer across the sea surface $\left(\left.\mathcal{F}\right|_{\text {sea }} ^{\text {air }}\right.$ for oxygen and carbon), and the fluxes across the seabed $\left(\left.\mathcal{F}\right|_{\text {ben }} ^{\text {pel }}\right)$. The actual numerical integration of these rates along with the advectiondiffusion processes that solve Eqs. (1) and (2) need to be addressed appropriately through an external driver as e.g. discussed in Butenschön et al. (2012).

\subsection{Nomenclature and units}

Pelagic state variables in ERSEM are concentrations and are referred to as $c_{\mathrm{p}}$. When indicating a specific class or type, they are denoted by upper-case letters $(P:$ phytoplankton; $Z$ : zooplankton; $B$ : bacteria; $R$ : organic matter; $O$ : gases; $N$ : nutrients), with the chemical component in the subscript in blackboard style $(\mathbb{C}$ : carbon; $\mathbb{N}$ : nitrogen; $\mathbb{P}$ : phosphorus; $\mathbb{S}$ : silicon; $\mathbb{F}$ : iron; with the exception of the chlorophyll $a$ components; which are distinguished by using $\mathcal{C}$, as chlorophyll $a$ is not a chemical element but a compound), and the specific type in the superscript, e.g. $\stackrel{\text { Pia }}{\mathbb{C}}_{\text {for }}$ diatom carbon. 


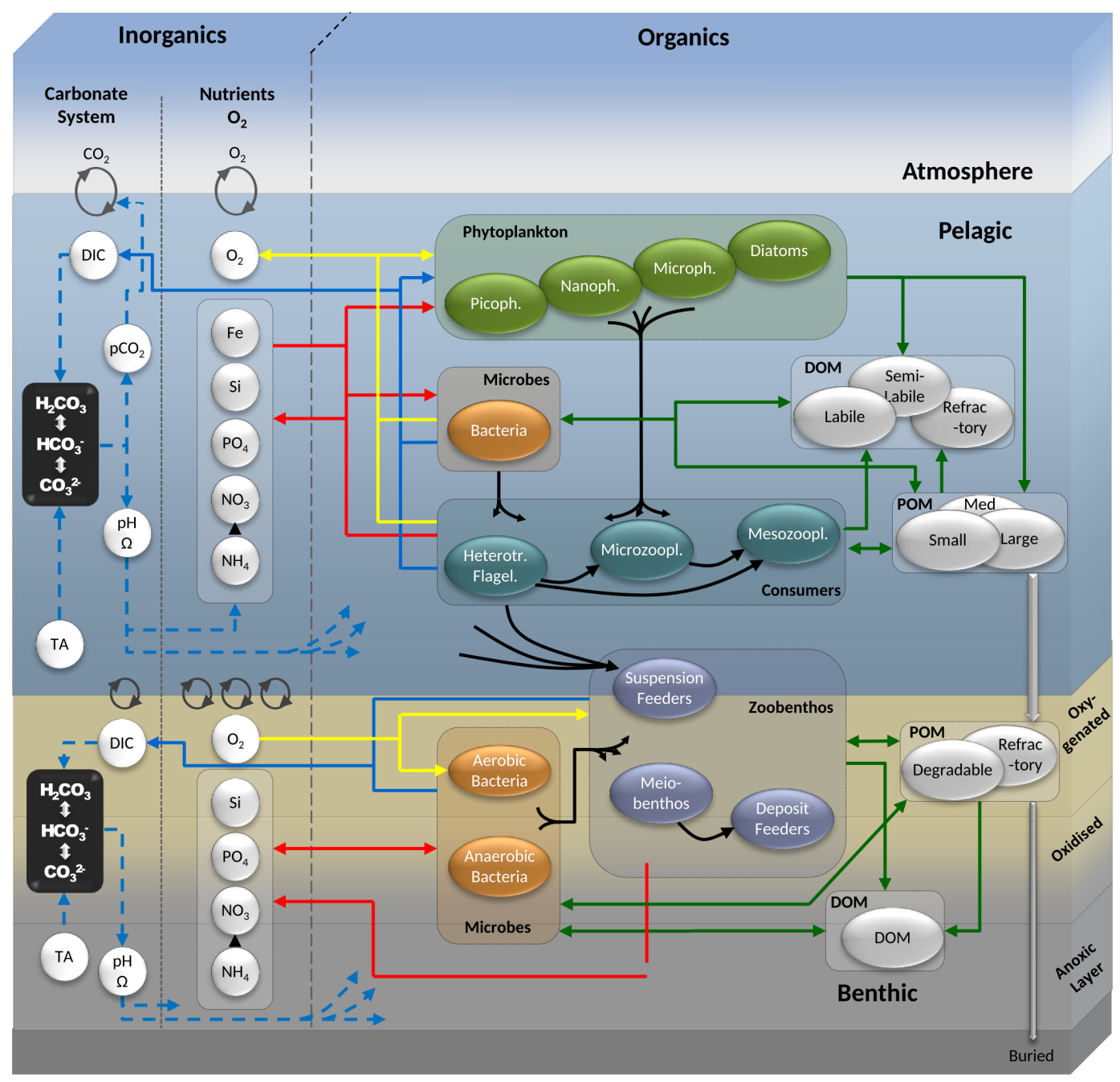

Figure 2. ERSEM schematic showing how model components interact with or influence each other. Blue connectors represent inorganic carbon fluxes, red represents nutrient fluxes, yellow represents oxygen, black represents predator-prey interactions, and green represents fluxes of non-living organics. Dashed arrows indicate the influence of carbonate system variables.

Correspondingly, benthic states use $c_{\mathrm{b}}$ for generic contents and the specific states $(H$ : bacteria; $Y$ : zoobenthos; $Q$ : organic matter; $G$ : gases; $K$ : nutrients; $D$ : states of vertical distribution). Primes (') mark available concentrations or contents to loss processes (see Sect. 2.3). Where equations are valid for more than one specific functional type $\chi, \psi$ and $\Psi$ are used as placeholders for functional types and the chemical components may be given as a comma separated list, implying that an equation is valid for all these components; for example, $P_{\mathbb{C}, \mathbb{N}, \mathbb{P}}^{\chi}$ represents the carbon, phosphorus, and nitrogen content of each phytoplankton type.

Parameters are represented by lower-case letters with $r$ for specific rates, $q$ for quotas or fractions, $l$ for limitation or regulating factors, $h$ for half-saturation constants, and $p$ for most others. Food preferences of predators for their prey are given as $\left.f_{\mathrm{pr}}\right|_{P} ^{Z}$, being the preference of predator $Z$ for food $P$.
Fluxes between state variables are given as $\left.\mathcal{F}\right|_{A} ^{B}$ for the flux from $A$ to $B$. Specific rates are notated using $\mathcal{S}$. Dynamic internal quotas of two components $A$ and $B$ are given by the notation $q_{A: B}$, e.g. $\stackrel{\text { dia }}{q}_{\mathbb{N}: \mathbb{C}}$ being the internal nitrogen to carbon quota of diatoms $\frac{P_{\mathbb{N}}}{P_{\mathbb{C}}}$. Derived quotas or fractions are given by a calligraphic $\mathcal{Q}$.

The coordinate system used describes the horizontal coordinates in $x$ and $y$, while the vertical coordinate is given by $z, 0$ at the sea surface increasing downwards. The corresponding velocity fields are given by $u, v$, and $w$. We refer to Cartesian coordinates in this publication for simplicity.

The sediment depth coordinate is given by $\zeta$, which is 0 at the sediment surface, increasing downwards.

All equations are given as scalar equations for a single pixel of the model domain. 
Rates of change of the biogeochemical state variables due to individual subprocesses or groupings of these are given as $\left.\frac{\partial \phi}{\partial t}\right|_{\text {subprocess }}$, where the following abbreviations are used for the subprocesses: bgc: biogeochemical fluxes; bur: burying; calc: calcification; decomp: decomposition; denit: denitrification; dis: dissolution; excr: excretion; mort: mortality; net: comprehensive net fluxes; nitr: nitrification; pred: predation; rel: release; remin: remineralization; resp: respiration; scav: scavenging; sed: sedimentation; upt: uptake.

In equations that hold for multiple functional groups or components, squared brackets are used for terms that are only valid for a single functional group or component.

Units in the model for all organic and inorganic nutrient concentrations are in $\mathrm{mmol} \mathrm{m}^{-3}$, with the exception of iron being in $\mu \mathrm{mol} \mathrm{m}{ }^{-3}$. All forms of organic carbon are in $\mathrm{mg} \mathrm{m}^{-3}$, while all species of inorganic carbon are in $\mathrm{mmol} \mathrm{m}^{-3}$, with the exception of the internal computations of the carbonate system, where they are converted to $\mu \mathrm{mol} \mathrm{kg}{ }^{-1}$. Corresponding benthic contents are twodimensional and consequently given in $\mathrm{mmol} \mathrm{m} \mathrm{m}^{-2}, \mathrm{mg} \mathrm{m}^{-2}$, and $\mu \mathrm{mol} \mathrm{m} \mathrm{m}^{-2}$. The penetration depth and depth horizons in the sediments are given in $\mathrm{m}$. Temperatures are generally considered in ${ }^{\circ} \mathrm{C}$, salinity in psu, seawater density in $\mathrm{kg} \mathrm{m}^{-3}$, and pressure in $\mathrm{Pa}$, with the exception of the internal calculations of the carbonate system where temperature is converted to absolute temperature in $\mathrm{K}$ and pressure to bar. Partial pressure of carbon dioxide is used in ppm.

\subsection{Dependencies on the physical environment}

Several processes in the model depend directly on the physical environment that the model states are exposed to.

- Metabolic processes depend on the seawater temperature.

- Primary production relies additionally on the photosynthetically active radiation (PAR) as energy input which should be computed from shortwave radiation at the sea surface $I_{\text {surf }}$, taking into account the attenuation coefficients given in Sect. 3.9. Note that the model requires the average light in each discrete model cell, which is not given by the light at the cell centre, but by the vertical integral of the light curve divided by the cell depth.

- Empirical regressions for alkalinity, saturation states, and chemical equilibrium coefficients of the carbonate system reactions require temperature $T$, salinity $S$, pressure $\mathrm{p}$, and density $\rho$ of the seawater.

- Air-sea fluxes of carbon dioxide and oxygen depend on temperature $T$ and the absolute wind speed $\boldsymbol{u}_{\text {wind }}$ near the sea surface.
- Deposition of organic matter on the seafloor and resuspension depend on the shear stress at the seafloor $\tau_{\text {bed }}$.

- The optional light attenuation model based on inherent optical properties requires the geographical coordinates of each model pixel and the current simulation date and time in order to compute the zenith angle.

\subsection{States and negativity control}

In order to avoid the occurrence of negative concentrations or contents in the integration process and reduce the vulnerability to numerical noise, all state variables include a lower buffer $\epsilon_{\mathrm{p}, \mathrm{b}}$, based on a carbon concentration of $0.01 \mathrm{mg} \mathrm{m}^{-3}$ modified adequately for the various state variables using reference stoichiometric quotas and unit conversions. This buffer is not accessible to the loss processes of the biogeochemical dynamics. Consequently all processes that diminish the biomass of each state are based on the available concentrations or contents given by $c_{\mathrm{p}, \mathrm{b}}^{\prime}=c_{\mathrm{p}, \mathrm{b}}-\epsilon_{\mathrm{p}, \mathrm{b}}$. These small resilient buffers additionally support the spawning of new biomass as soon as favourable conditions occur, similar to the low overwintering biomass limits in Fennel (1995).

Note that when calculating the overall budgets of a domain, these background concentrations should be subtracted in order to give adequate results.

\section{The pelagic system}

In its current form the pelagic part of ERSEM comprises four functional types for primary producers, originally defined as diatoms, nanoflagellates, picophytoplankton, and dinoflagellates. This classification was historically coined for the North Sea, but has since been widened to a broader interpretation almost exclusively based on the single trait size (with the exception of the requirement of silicate by diatoms and an implicit calcification potential of nanoflagellates), leading to the classes of picophytoplankton, nanophytoplankon, microphytoplankton, and diatoms. Similarly the zooplankton pool is divided into heterotrophic nanoflagellates, microzooplankton, and mesozooplankton. Particulate organic matter is treated in three size classes (small, medium, and large) in relation to its origin. Dissolved organic matter is distinguished according to its decomposition timescales into a labile dissolved inorganic state and semi-labile and semirefractory carbon (see Sect. 3.3.1).

The inorganic state variables of the pelagic model are dissolved oxidized nitrogen, ammonium, phosphate, silicic acids, dissolved inorganic iron, dissolved inorganic carbon, dissolved oxygen, and calcite. In addition, the model holds a state variable for alkalinity subject to fluctuations generated from the modelled biogeochemical processes (see Sect. 3.8 and Artioli et al., 2012). The complete list of pelagic state variables is given in Table 1. 
Table 1. Pelagic functional types and their components (squared brackets indicate optional states) - chemical components: $\mathbb{C}$ carbon, $\mathbb{N}$ nitrogen, $\mathbb{P}$ phosphorus, $\mathbb{F}$ iron, $\mathbb{S}$ silicate, $\mathcal{C}$ chlorophyll $a$.

\begin{tabular}{|c|c|c|}
\hline Symbol & Code & Description \\
\hline \multicolumn{3}{|l|}{ pico } \\
\hline$P_{\mathbb{C}, \mathbb{N}, \mathbb{P}[, \mathbb{F}], \mathcal{C}}$ & $\mathrm{P} 3 \mathrm{c}, \mathrm{n}, \mathrm{p}[\mathrm{f}] \mathrm{f}, \mathrm{Ch} 13$ & Picophytoplankton $(<2 \mu \mathrm{m})$ \\
\hline$P_{\mathbb{C}, \mathbb{N}, \mathbb{P}[, \mathbb{F}], \mathcal{C}}$ & $\mathrm{P} 2 \mathrm{c}, \mathrm{n}, \mathrm{p}[, \mathrm{f}], \mathrm{Ch} 12$ & Nanophytoplankton $(2-20 \mu \mathrm{m})$ \\
\hline$\stackrel{\text { micro }}{P} \mathbb{C}, \mathbb{N}, \mathbb{P}[, \mathbb{F}], \mathcal{C}$ & $\mathrm{P} 4 \mathrm{c}, \mathrm{n}, \mathrm{P}[\mathrm{f}], \mathrm{Ch} 14$ & Microphytoplankton (> $20 \mu \mathrm{m})$ \\
\hline$\stackrel{\mathrm{dia}}{P}_{\mathbb{C}, \mathbb{N}, \mathbb{P}[, \mathbb{F}], \mathbb{S}, \mathcal{C}}$ & $P 1 c, n, P[, f], C h 11$ & Diatoms \\
\hline${ }_{Z}^{\operatorname{HET}} \mathbb{C}, \mathbb{N}, \mathbb{P}$ & $\mathrm{z} 6 \mathrm{c}, \mathrm{n}, \mathrm{p}$ & Heterotrophic flagellates \\
\hline $\begin{array}{l}\text { MICRO } \\
\qquad Z \mathbb{C}, \mathbb{N}, \mathbb{P}\end{array}$ & $\mathrm{Z} 5 \mathrm{c}, \mathrm{n}, \mathrm{p}$ & Microzooplankton \\
\hline $\begin{array}{l}\mathrm{MESO} \\
Z_{\mathbb{C}}\end{array}$ & $\mathrm{Z} 4 \mathrm{C}$ & Mesozooplankton \\
\hline $\begin{array}{l}B_{\mathbb{C}, \mathbb{N}, \mathbb{P}} \\
\mathrm{lab}\end{array}$ & $B 1 c, n, p$ & Heterotrophic bacteria \\
\hline$R_{\mathbb{C}, \mathbb{N}, \mathbb{P}}$ & $\mathrm{R} 1 \mathrm{c}, \mathrm{n}, \mathrm{p}$ & Labile dissolved organic matter \\
\hline$R_{\mathbb{C}}$ & $\mathrm{R} 2 \mathrm{C}$ & Semi-labile organic matter \\
\hline$R_{\mathbb{C}}$ & R3c & Semi-refractory organic matter \\
\hline$\stackrel{\text { small }}{R} \mathbb{C}, \mathbb{N}, \mathbb{P}[, \mathbb{F}]$ & $\mathrm{R} 4 \mathrm{c}, \mathrm{n}, \mathrm{p}[\mathrm{f} \mathrm{f}]$ & Small particulate organic matter \\
\hline$\stackrel{{ }_{\text {large }}}{R} \mathbb{C}, \mathbb{N}, \mathbb{P}[, \mathbb{F}], \mathbb{S}$ & $\mathrm{R} 6 \mathrm{c}, \mathrm{n}, \mathrm{p}[\mathrm{f} \mathrm{f}], \mathrm{s}$ & Medium size particulate organic matter \\
\hline$R_{\mathbb{C}, \mathbb{N}, \mathbb{P}, \mathbb{S}}$ & $\mathrm{R} 8 \mathrm{c}, \mathrm{n}, \mathrm{p}, \mathrm{s}$ & Large particulate organic matter \\
\hline$\left[\begin{array}{l}\text { calc } \\
L_{\mathbb{C}}\end{array}\right]$ & {$[\mathrm{L} 2 \mathrm{C}]$} & Calcite \\
\hline$O_{\mathbb{O}}$ & 020 & Dissolved oxygen \\
\hline$O_{\mathbb{C}}$ & $03 c$ & Dissolved inorganic carbon (DIC) \\
\hline$N_{\mathbb{P}}$ & $\mathrm{N} 1 \mathrm{p}$ & Phosphate \\
\hline $\begin{array}{l}\mathrm{ox}_{\mathbb{N}} \\
\mathrm{amm}\end{array}$ & N3n & Oxidized nitrogen \\
\hline$N_{\mathbb{N}}$ & $\mathrm{N} 4 \mathrm{n}$ & Ammonium \\
\hline$N_{\mathbb{S}}$ & N5s & Silicate \\
\hline$\left[N_{\mathbb{F}}\right]$ & {$[N 7 f]$} & Dissolved iron \\
\hline$\left[\mathbb{A}_{\text {bio }}\right]$ & [bioAlk] & Bioalkalinity \\
\hline
\end{tabular}

The recently implemented iron cycle (following largely the implementation of Vichi et al., 2007) and the silicate cycle are abbreviated for simplicity; their pathways by-pass the predators and decomposers by turning grazing of phytoplankton iron or silicate directly into detritus and remineralizing iron implicitly from detritus into the dissolved inorganic form, while silicate is not remineralized in the water column. Chlorophyll $a$ takes a special role in between the chemical components of the model: being a compound of other elements, it is not strictly conserved by the model equations but rather derived from assimilation of carbon and subsequent decomposition of organic compounds. The addition of chlorophyll $a$ states to the model allows for dynamic chlorophyll $a$ to carbon relationships in the photosynthesis description and a more accurate comparison to observations of biomass or chlorophyll $a$.

The growth dynamics in the model are generally based on mass-specific production and loss equations that are ex- pressed in the currency of each chemical component, regulated and limited by the availability of the respective resources.

\subsection{Primary producers}

The phytoplankton dynamics are modelled for each phytoplankton type as a net result of source and loss processes (Varela et al., 1995). The carbon and chlorophyll $a$ component is given by uptake in the form of gross primary production and the losses through excretion, respiration, predation by zooplankton, and mortality in the form of lysis, while the nutrient content is balanced by uptake, release, predation, and mortality in the form of lysis: 


$$
\begin{aligned}
\left.\frac{\partial \stackrel{\chi}{P}}{\partial t}\right|_{\mathbb{C}, \mathcal{C}} & =\left.\frac{\partial \stackrel{\chi}{P}_{\mathbb{C}, \mathcal{C}}}{\partial t}\right|_{\mathrm{gpp}}-\left.\frac{\partial \stackrel{\chi}{P}_{\mathbb{C}, \mathcal{C}}}{\partial t}\right|_{\text {excr }}-\left.\frac{\partial \stackrel{\chi}{P}_{\mathbb{C}, \mathcal{C}}}{\partial t}\right|_{\text {resp }} \\
& -\left.\frac{\partial \stackrel{\chi}{P}_{\mathbb{C}, \mathcal{C}}}{\partial t}\right|_{\text {pred }}-\left.\frac{\partial \stackrel{\chi}{P}_{\mathbb{C}, \mathcal{C}}}{\partial t}\right|_{\text {mort }},
\end{aligned}
$$

$$
\begin{aligned}
& \left.\frac{\partial \stackrel{\chi}{P}_{\mathbb{N}}, \mathbb{P}, \mathbb{F}[, \mathbb{S}]}{\partial t}\right|_{\text {bgc }}=\left.\frac{\partial \stackrel{\chi}{P}_{\mathbb{N}, \mathbb{P}, \mathbb{F}[, \mathbb{S}]}}{\partial t}\right|_{\text {upt }}-\left.\frac{\partial \stackrel{\chi}{P}_{\mathbb{N}, \mathbb{P}, \mathbb{F}[, \mathbb{S}]}}{\partial t}\right|_{\text {rel }} \\
& -\left.\frac{\partial \stackrel{x}{P}_{\mathbb{N}, \mathbb{P}, \mathbb{F}, \mathbb{S}[}}{\partial t}\right|_{\text {pred }}-\left.\frac{\partial \stackrel{x}{P_{\mathbb{N}, \mathbb{P}, \mathbb{F}[, \mathbb{S}]}}}{\partial t}\right|_{\text {mort }},
\end{aligned}
$$

with $\chi$ in (pico, nano, micro, dia) and where the silicate component $(\mathbb{S})$ is only active for diatoms.

The formulation of photosynthesis combines the form originally presented in Baretta-Bekker et al. (1997) for the balance of carbon assimilation, excretion, and respiration with the negative exponential light harvesting model based on Jassby and Platt (1976), Platt et al. (1982), and Geider et al. (1997) in order to describe the total specific carbon fixation. In this formulation the gross carbon assimilation is assumed to be independent of nitrogen and phosphorus. Total gross primary production (GPP) is assumed to be composed of a fraction which is assimilated (cellular GPP) through photosynthesis and a fraction which is not utilizable, e.g. due to nutrient limitation, and excreted. A similar approach can be found in Falkowski and Raven (2007). The idea behind this assumption is that nutrient (or specifically nitrogen and phosphorus) limitation affects more the assimilation of newly fixed carbon into cellular biomass (assimilation) than the photosynthesis itself.

Phytoplankton mass-specific gross primary production is then computed as

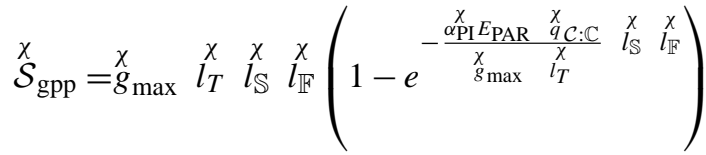

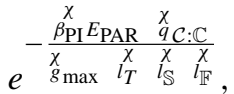

based on the formulation by Geider et al. (1997) modified for photoinhibition according to Blackford et al. (2004). The symbols in this equation represent the chlorophyll $a$ to carbon quota of each functional type $\stackrel{\chi}{q}_{\mathcal{C}: \mathbb{C}}=\stackrel{\chi}{P}_{\mathcal{C}} / \stackrel{\chi}{P}_{\mathbb{C}}$, the metabolic response to temperature $\stackrel{\chi}{l}_{T}$ (see Eq. 239), and the silicate and iron limitation factors $\stackrel{\chi}{l}_{\mathbb{S}, \mathbb{F}} \in[0,1]$ (see Eqs. 243 and 244). The $\stackrel{\chi}{g}_{\max }$ are the maximum potential photosynthetic rate parameters in unlimiting conditions at reference temperature. Note that these are different to the maximum potential growth rates usually retrieved in physiological experiments (e.g. in the work of Geider et al., 1997) or measured at sea, in that they are exclusive upper bounds of the specific growth rate function. In fact, the products of the exponential terms in Eq. (5) have a maximum of

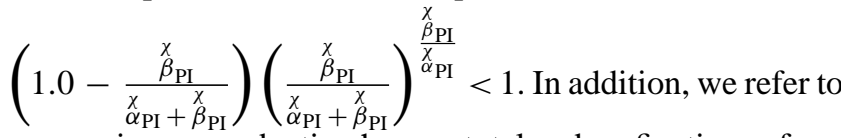
gross primary production here as total carbon fixation, a fraction of which is directly excreted. Other parameters are the initial slope $\stackrel{\chi}{\alpha}$ PI and the photoinhibition parameter $\stackrel{\chi}{\beta}{ }_{\text {PI }}$ of the light saturation curve (Platt et al., 1982).

A fraction of the specific gross production is directly excreted to the dissolved organic carbon (DOC) pool as a fixed fraction $\stackrel{\chi}{q}_{\text {excr }}$ augmented according to the combined nitrogen and phosphorus limitation up to the total gross production:

$\stackrel{\chi}{\mathcal{Q}}_{\text {excr }}=\stackrel{\chi}{q}_{\text {excr }}+\left(1-\stackrel{\chi}{l}_{\langle\mathbb{N} \mathbb{P}\rangle}\right)\left(1-\stackrel{\chi}{q}_{\text {excr }}\right)$,

where $\stackrel{\chi}{l}_{\langle\mathbb{N P}\rangle}$ is the combined nitrogen-phosphorus limitation factor defined in Eq. (242), based on the internal nutrient to carbon quotas according to Droop (1974).

The second generic sink term is given by lysis, which occurs proportionally to the current biomass by the constant specific rate $\stackrel{\chi}{r}$ mort augmented by nutrient stress according to

$\stackrel{\chi}{\mathcal{S}}_{\text {mort }}=\frac{1}{\min \left(\stackrel{\chi}{l}_{\langle\mathbb{N P P}\rangle}, l_{\mathbb{S}}^{\chi}\right)+0.1} \stackrel{\chi}{r}_{\text {mort }}$.

The carbon and chlorophyll $a$ dynamics of each phytoplankton type in Eq. (3) are then specified by the following terms: carbon is assimilated according to

$\left.\frac{\partial \stackrel{\chi}{P}}{\partial t}\right|_{\mathbb{g p p}}=\stackrel{\chi}{\mathcal{S}}$ gpp $\stackrel{\chi}{P}_{\mathbb{C}}$.

The synthesis rate of chlorophyll $a$ is given by

$\left.\frac{\partial \stackrel{\chi}{P}_{\mathcal{C}}}{\partial t}\right|_{\text {gpp }}=\stackrel{\chi}{l}_{\langle\mathbb{N} P} \quad \stackrel{\chi}{\varphi} \stackrel{\chi}{\mathcal{S}}_{\text {gpp }} \stackrel{\chi}{P}_{\mathbb{C}}$,

where $\stackrel{\chi}{\varphi}$ is the ratio of chlorophyll $a$ synthesis to carbon fixation under nutrient replete conditions. It is given by

$\stackrel{\chi}{\varphi}=\left(\stackrel{\chi}{q}_{\varphi \max }-q_{\min }: \mathbb{C}\right) \frac{\stackrel{\chi}{\mathcal{S}}_{\mathrm{gpp}}}{\alpha_{\mathrm{PI}}^{\chi} E_{\mathrm{PAR}} \stackrel{\chi}{q}_{\mathcal{C}: \mathbb{C}}}+q_{\min _{\mathcal{C}: \mathbb{C}}}$,

where $\stackrel{\chi}{q}_{\varphi \text { max }}$ are the maximum achievable chlorophyll $a$ to carbon quotas for each type; $q_{\min _{\mathcal{C}: \mathbb{C}}}$ is the minimum chlorophyll $a$ to carbon quota.

This formulation differs from the original formulation of Geider et al. (1997) in its asymptotic limit of the carbon to 
chlorophyll $a$ synthesis at high PAR. In the original formulation the ratio is unbound, while in this formulation it is bound by the inverse minimum chlorophyll $a$ to carbon ratio $q_{\min }: \mathbb{C}$ in order to avoid excessive quotas not observed in nature.

As opposed to the previous formulation of Blackford et al. (2004), the relative synthesis of chlorophyll $a$ is directly limited by the internal nutrient quota in order to compensate for the enhanced demand required to maintain the cell structure, leading to a reduced investment in the light harvesting capacity.

The excretion of phytoplankton in terms of carbon and chlorophyll $a$ is given by

$$
\left.\frac{\partial \stackrel{\chi}{P}_{\mathbb{C}, \mathcal{C}}}{\partial t}\right|_{\text {excr }}=\left.\stackrel{\chi}{\mathcal{Q}}_{\text {excr }} \frac{\partial \stackrel{\chi}{P}_{\mathbb{C}, \mathcal{C}}}{\partial t}\right|_{\mathrm{gpp}} .
$$

Respiration of phytoplankton is split into respiration at rest, that is, proportionally to the current biomass by the constant specific rate $\stackrel{\chi}{r}_{\text {resp }}$ complemented with an activityrelated term that is a fraction $\stackrel{\chi}{q}$ aresp of the assimilated amount of biomass per time unit after excretion:

$$
\begin{aligned}
\left.\frac{\partial \stackrel{\chi}{P}_{\mathbb{C}, \mathcal{C}}}{\partial t}\right|_{\text {resp }} & \stackrel{\chi}{r} \underset{\text { resp }}{ } \stackrel{\stackrel{\chi}{P}^{\prime}}{\mathbb{C}, \mathcal{C}} \\
& +\stackrel{\chi}{q}_{\text {aresp }}\left(\left.\frac{\partial \stackrel{\chi}{P}}{\partial t}\right|_{\mathbb{C}, \mathcal{C}}-\left.\frac{\partial \stackrel{\chi}{P}_{\mathbb{C}, \mathcal{C}}}{\partial t}\right|_{\text {excr }}\right) .
\end{aligned}
$$

The losses of phytoplankton by lysis are given by

$$
\left.\frac{\partial \stackrel{\chi}{P}_{\mathbb{C}, \mathcal{C}}}{\partial t}\right|_{\text {mort }}=\stackrel{\chi}{\mathcal{S}}_{\text {mort }}{\stackrel{\chi}{P^{\prime}}}_{\mathbb{C}, \mathcal{C}},
$$

while the individual terms of loss through predation of predator $\Psi$ in

$$
\left.\frac{\partial \stackrel{\chi}{P}_{\mathbb{C}, \mathcal{C}}}{\partial t}\right|_{\text {pred }}=\left.\sum_{\Psi} \mathcal{F}\right|_{P} ^{\Psi} \underset{P}{\Psi} \stackrel{\chi}{P^{\prime}} \underset{\mathbb{C}, \mathcal{C} .}{ }
$$

are specified in the sections on the respective predators in Eqs. (31) and (177).

Nutrient uptake of nitrogen, phosphorus, and iron is regulated by the nutrient demand of the phytoplankton group, limited by the external availability. Excretion is modelled as the disposal of non-utilizable carbon in photosynthesis, while the release of nutrients is limited to the regulation of the internal stoichiometric ratio. This approach is consistent with observations that nutrient excretion plays a minor role in the phytoplankton fluxes (Puyo-Pay et al., 1997). Consequently, demand of nutrients may be positive or negative in sign in relation to the levels of the internal nutrient storages and the balance between photosynthesis and carbon losses, so that

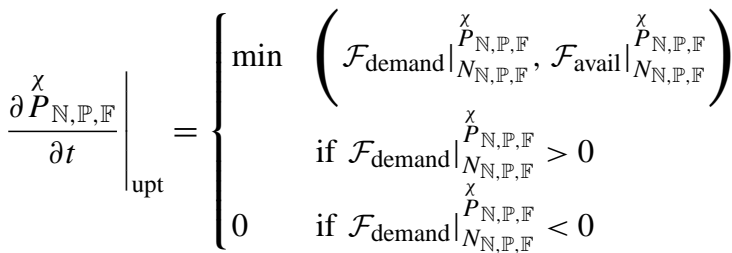

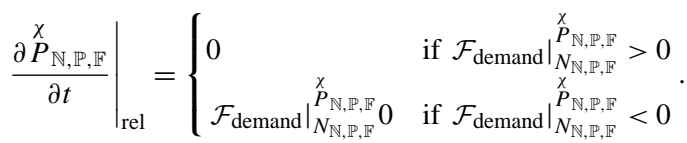

Nutrient demand (with the exception of silicate) is computed from assimilation demand at maximum quota $\stackrel{\chi}{q}_{\text {max }_{\mathbb{N}, \mathbb{P}, \mathbb{C}: \mathbb{C}}}$ complemented by a regulation term relaxing the internal quota towards the maximum quota and compensating for rest respiration:

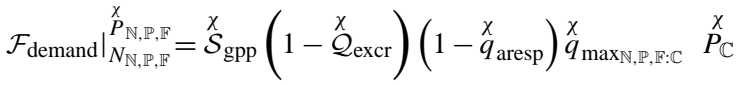

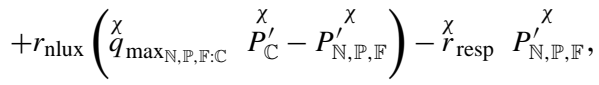

where $r_{\text {nlux }}$ is the rate of nutrient luxury uptake towards the maximum quota.

Note that these terms may turn negative when rest respiration exceeds the effective assimilation rate $\stackrel{\chi}{\mathcal{S}}_{\text {gpp }}\left(1-\stackrel{\chi}{\mathcal{Q}}_{\text {excr }}\right)\left(1-\stackrel{\chi}{q}_{\text {aresp }}\right) \stackrel{\chi}{P_{\mathbb{C}}}$ or the internal nutrient content exceeds the maximum quota, resulting in nutrient excretion in dissolved inorganic from. The maximum quota for nitrogen and phosphorus may exceed the optimal quota, allowing for luxury storage, while it is identical to the optimum quota for iron and silicate.

The uptake is capped at the maximum achievable uptake depending on the nutrient affinities $\stackrel{\chi}{r}_{\text {aff }}{ }_{\mathbb{P}, \mathbb{R}, n, a}$ and the external dissolved nutrient concentrations:

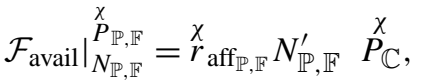

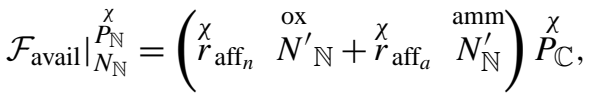

where the nitrogen need is satisfied by uptake in oxidized and reduced form in relation to the respective affinities ${ }^{2}$ and external availability.

This purely linear formulation of maximum uptake proportional to the affinity is in contrast to the more widely used saturation assumption of Michaelis-Menten type (Aksnes and Egge, 1991). It is justified here as ERSEM treats phytoplankton in pools of functional groups, rather than as individual species with defined saturation characteristics (Franks, 2009).

\footnotetext{
${ }^{2}$ Note that the dimensions of these are $\left[\right.$ volume $^{1} \cdot$ mass $^{-1} \cdot$ time $\left.^{-1}\right]$ as opposed to $\left[\right.$ time $\left.^{-1}\right]$ as for most other rates.
} 
Lysis and predation losses are computed analogously to the carbon component:

$$
\begin{aligned}
& \left.\frac{\partial \stackrel{\chi}{P}_{\mathbb{N}, \mathbb{P}, \mathbb{F}}}{\partial t}\right|_{\text {mort }}=\stackrel{\chi}{\mathcal{S}}_{\text {mort }} \stackrel{\chi}{P^{\prime}} \underset{\mathbb{N}, \mathbb{P}, \mathbb{F}}{ }, \\
& \left.\frac{\partial \stackrel{\chi}{P_{\mathbb{N}, \mathbb{P}, \mathbb{F}}}}{\partial t}\right|_{\text {pred }}=\left.\sum_{\Psi} \mathcal{F}\right|_{P} ^{\Psi} \underset{P}{\Psi} \stackrel{\chi}{P^{\prime}}, \mathbb{N}, \mathbb{P}, \mathbb{F} .
\end{aligned}
$$

The variability of the internal silicate quota of diatoms reported in the literature is small and there is little evidence of luxury uptake capacity for this element (Brzezinski, 1985; Moore et al., 2013). The silicate dynamics of diatoms are therefore modelled by a simple relaxation towards the optimal quota given by the equations

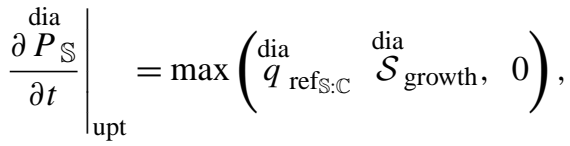

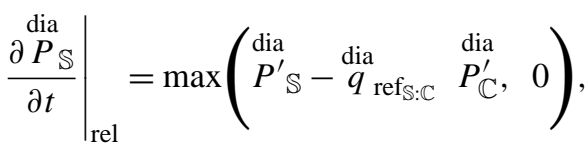

$$
\begin{aligned}
& \left.\frac{\partial \stackrel{\mathrm{dia}}{P}_{\mathbb{S}}}{\partial t}\right|_{\text {mort }}=\stackrel{\mathrm{dia}}{\mathcal{S}}_{\text {mort }} \stackrel{\text { dia }}{P^{\prime}}, \\
& \left.\frac{\partial P_{\mathbb{S}}^{\text {dia }}}{\partial t}\right|_{\text {pred }}=\left.\sum_{\Psi} \mathcal{F}\right|_{P} ^{\Psi i a} \underset{S}{\Psi} \stackrel{\text { dia }}{P^{\prime}}
\end{aligned}
$$

where $\stackrel{\text { dia }}{q}_{\text {ref }}$ is the reference silicate to carbon quota of diatoms.

A formulation to model the impact of an increased atmospheric $p_{\mathrm{CO}_{2}}$ on phytoplankton carbon uptake that was introduced in Artioli et al. (2014b) is available via the CENH preprocessing option. In this case gross carbon uptake (Eq. 8) and activity respiration (the second term in Eq. 12) are enhanced by the factor $\gamma_{\text {enh } \mathbb{C}}$ defined as

$\gamma_{\mathrm{enh} \mathbb{C}}=1.0+\left(\mathrm{p}_{\mathrm{CO}_{2}}-379.48\right) \times 0.0005$,

where $\mathrm{PCO}_{2}$ has the unit ppm.

\subsection{Predators}

Predator dynamics are largely based on the descriptions of Baretta-Bekker et al. (1995), Broekhuizen et al. (1995), and Heath et al. (1997) described by the equations

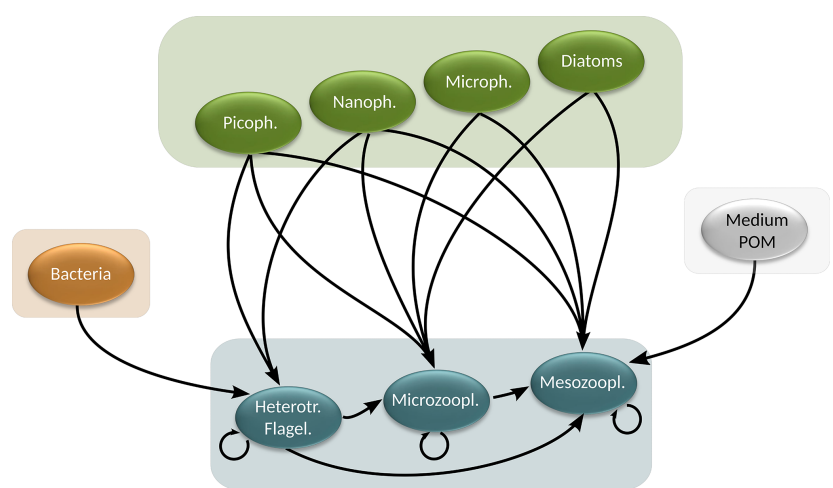

Figure 3. Pelagic predators and their prey.

$$
\begin{aligned}
& \left.\frac{\partial \stackrel{\chi}{Z}_{\mathbb{C}}}{\partial t}\right|_{\text {bgc }}=\left.\frac{\partial \stackrel{\chi}{Z}_{\mathbb{C}}}{\partial t}\right|_{\text {upt }}-\left.\frac{\partial \stackrel{\chi}{Z}_{\mathbb{C}}}{\partial t}\right|_{\text {excr }}-\left.\frac{\partial \stackrel{\chi}{Z}_{\mathbb{C}}}{\partial t}\right|_{\text {resp }} \\
& -\left.\frac{\partial \stackrel{\chi}{Z}_{\mathbb{C}}}{\partial t}\right|_{\text {pred }}-\left.\frac{\partial \stackrel{\chi}{Z}_{\mathbb{C}}}{\partial t}\right|_{\text {mort }}, \\
& \left.\frac{\partial \stackrel{\chi}{Z}_{\mathbb{N}, \mathbb{P}}}{\partial t}\right|_{\text {bgc }}=\left.\frac{\partial \stackrel{\chi}{Z}_{\mathbb{N}, \mathbb{P}}}{\partial t}\right|_{\text {upt }}-\left.\frac{\partial \stackrel{\chi}{Z}_{\mathbb{N}, \mathbb{P}}}{\partial t}\right|_{\text {excr }}-\left.\frac{\partial \stackrel{\chi}{Z}_{\mathbb{N}, \mathbb{P}}}{\partial t}\right|_{\text {rel }} \\
& -\left.\frac{\partial \stackrel{\chi}{Z}_{\mathbb{N}, \mathbb{P}}}{\partial t}\right|_{\text {pred }}-\left.\frac{\partial \stackrel{\chi}{Z}_{\mathbb{N}, \mathbb{P}}}{\partial t}\right|_{\text {mort }} .
\end{aligned}
$$

Note that the iron and silicate cycles are simplified in a way that the iron/silicate content of phytoplankton subject to predation is directly turned into particulate organic matter (see Eqs. 72 and 73).

The pelagic predators considered in ERSEM are composed of three size classes of zooplankton categorized as heterotrophic flagellates, microzooplankton, and mesozooplankton. According to size, these are capable of predating on different prey types, including cannibalism as illustrated in Fig. 3.

The total prey available to each zooplankton type $\chi$ are composed of the individual prey types $\psi$ using type II Michaelis-Menten-type uptake capacities (Chesson, 1983; Gentleman et al., 2003) as

$$
\stackrel{\chi}{\operatorname{Pr}} \mathbb{C}, \mathbb{N}, \mathbb{P}^{\prime}=\left.\sum_{\psi} f_{\mathrm{pr}}\right|_{\psi} ^{\stackrel{\chi}{Z}} \frac{\psi_{\mathbb{C}}^{\prime}}{\psi_{\mathbb{C}}^{\prime}+h_{\min }} \psi_{\mathbb{C}, \mathbb{N}, \mathbb{P}}^{\prime},
$$

where $\left.f_{\mathrm{pr}}\right|_{\psi} ^{\chi}$ are the food preferences and $\stackrel{\chi}{h_{\min }}$ is a food halfsaturation constant reflecting the detection capacity of predator $\chi$ of individual prey types. 
The prey mass-specific uptake capacity for each zooplankton type $\chi$ is then given by

$$
\stackrel{\chi}{\mathcal{S}}_{\text {growth }}=\stackrel{\chi}{g}_{\max } l_{T} \frac{\stackrel{\chi}{Z}_{\mathbb{C}}}{\operatorname{Pr}_{\mathbb{C}}+h_{\text {up }}},
$$

where $\stackrel{\chi}{g}_{\text {max }}$ is the maximum uptake capacity of each type at the reference temperature, $l_{T}$ is the metabolic temperature response (Eq. 239), and $h_{\text {up }}^{\chi}$ is a predation efficiency constant limiting the chances of encountering prey. Introducing the prey mass-specific fluxes from prey $\psi$ to predator $\chi$

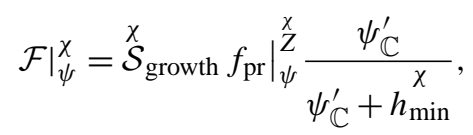

the zooplankton uptake can then be written as

$$
\left.\frac{\partial \stackrel{\chi}{Z}_{\mathbb{C}, \mathbb{N}, \mathbb{P}}}{\partial t}\right|_{\text {upt }}=\left.\sum_{\psi} \mathcal{F}\right|_{\psi} ^{\stackrel{\chi}{Z}} \psi_{\mathbb{C}, \mathbb{N}, \mathbb{P}}^{\prime}
$$

This formulation is similar to the approach used in Fasham et al. (1990), but introduces additional Michaelis-Menten terms for individual prey types. The purpose here is to include sub-scale effects of pooling as prey of different types can be assumed to be distributed in separate patches in the comparatively large cell volume. Consequently, individual prey patches below a certain size are less likely to be grazed upon compared to the larger patches, which is expressed by the $\stackrel{\chi}{h_{\text {min }}}$ parameter.

Note that in contrast to previous parametrizations, we now normalize the sum of the food preferences for each predator $\stackrel{\chi}{Z}$ to

$$
\left.\sum_{\psi} f_{\mathrm{pr}}\right|_{\psi} ^{\chi}=1
$$

as non-normalized preferences lead to a hidden manipulation of the predation efficiency and at low prey concentrations of the maximum uptake capacity $\stackrel{\chi}{g}_{\max }$.

The ingestion and assimilation of food by the predators is subject to inefficiencies that, given the wide diversity of uptake mechanisms within the zooplankton pools, is for simplicity taken as a fixed proportion of the gross uptake $1-\stackrel{\chi}{q}_{\text {eff }}$. These losses are attributed to the excretion of faeces as a constant fraction $\left(\stackrel{\chi}{q}_{\text {excr }}\right)$ and activity costs in form of enhanced respiration $\left(1-\stackrel{\chi}{q}_{\text {excr }}\right)$.

The excretion term in Eq. (27) is then given by

$$
\left.\frac{\partial \stackrel{\chi}{Z}_{\mathbb{C}, \mathbb{N}, \mathbb{P}}}{\partial t}\right|_{\text {excr }}=\left.\left(1-\stackrel{\chi}{q}_{\text {eff }}\right) \stackrel{\chi}{q}_{\text {excr }} \frac{\partial \stackrel{\chi}{Z}_{\mathbb{C}, \mathbb{N}, \mathbb{P}}}{\partial t}\right|_{\text {upt }} .
$$

Respiration losses are composed of the activity costs and a basal respiration term required for maintenance and are hence proportional to the current biomass by the constant factor $\stackrel{\chi}{r}_{\text {resp }}$ multiplied by the metabolic temperature response (Eq. 239):

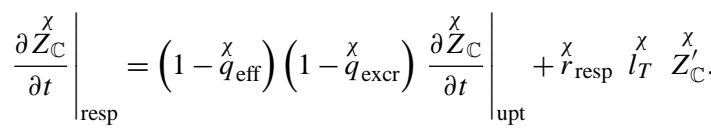

This simple formulation of assimilation losses is closely related to the phytoplankton losses described in the previous section following the concept of the standard organism (Baretta et al., 1995) pending a better understanding of the underlying physiological mechanisms (Anderson et al., 2013).

Nitrogen and phosphorus are released, regulating the internal stoichiometric quota:

$\left.\frac{\partial \stackrel{\chi}{Z}_{\mathbb{N}, \mathbb{P}}}{\partial t}\right|_{\text {rel }}=\min \left(0,{\stackrel{\chi}{Z^{\prime}}}_{\mathbb{N}, \mathbb{P}}-\stackrel{\chi}{q}_{\mathbb{N}, \mathbb{P}: \mathbb{C}}{\stackrel{\chi}{Z^{\prime}} \mathbb{C}}\right) \stackrel{\chi}{r}_{\text {rel }, \mathbb{P}}$,

where $\stackrel{\chi}{r}_{\text {relP, } \mathbb{N}}$ are the relaxation rates of release into dissolved inorganic form (see Eqs. 109 and 112).

Mortality is proportional to biomass based on a basal rate $\stackrel{\chi}{p}_{\text {mort }}$ enhanced up to $\stackrel{\chi}{p}_{\text {mort }} \mathbb{O}+\stackrel{\chi}{p}_{\text {mort }}$ under oxygen limitation $\stackrel{\chi}{l_{\mathbb{O}}}($ Eq. 249) as

$\frac{\partial{\stackrel{\chi}{Z_{\mathbb{C}}, \mathbb{N}, \mathbb{P}}}_{\partial t}}{\left.\partial\right|_{\text {mort }}}=\left(\left(1-{\stackrel{\chi}{l_{\mathbb{O}}}}\right) \stackrel{\chi}{p}_{\text {mort } \mathbb{O}}+\stackrel{\chi}{p}_{\text {mort }}\right){\stackrel{\chi}{Z^{\prime}}}_{\mathbb{C}, \mathbb{N}, \mathbb{P}}$

Biomass lost to other predators $\Psi$ is computed as

$$
\left.\frac{\partial \stackrel{\chi}{Z}_{\mathbb{C}, \mathbb{N}, \mathbb{P}}}{\partial t}\right|_{\text {pred }}=\left.\sum_{\Psi} \mathcal{F}\right|_{Z} ^{\Psi} \stackrel{\chi}{Z}^{\prime}{ }_{\mathbb{C}, \mathbb{N}, \mathbb{P}} .
$$

\section{Mesozooplankton}

The top-level predator mesozooplankton takes a special role in the predator group in three respects.

- Its internal nutrient to carbon quota is assumed fixed (Gismervik, 1997; Walve and Larsson, 1999).

- It is capable of scavenging on particulate organic matter.

- At low prey it can enter a hibernation state (optional) at which its maintenance metabolism is reduced (Blackford et al., 2004).

The resulting overall balance of the meszooplankton dynamics is in principle identical to the other zooplankton types (Eqs. 27 and 28) with the exception of an additional release 
term for carbon in order to maintain the fixed internal stoichiometric quota:

$$
\begin{aligned}
\left.\frac{\partial Z_{\mathbb{C}}}{\partial t}\right|_{\text {bgc }} & =\left.\frac{\partial Z_{\mathbb{C}}}{\partial t}\right|_{\text {upt }}-\left.\frac{\partial Z_{\mathbb{C}}}{\partial t}\right|_{\text {excr }}-\left.\frac{\partial Z_{\mathbb{C}}}{\partial t}\right|_{\text {resp }} \\
& -\left.\frac{\partial Z_{\mathbb{C}}}{\partial t}\right|_{\text {rel }}-\left.\frac{\partial Z_{\mathbb{C}}}{\partial t}\right|_{\text {pred }}-\left.\frac{\partial Z_{\mathbb{C}}}{\partial t}\right|_{\text {mort }},
\end{aligned}
$$

$$
\begin{aligned}
\left.\frac{\partial Z_{\mathbb{N}, \mathbb{P}}^{\mathrm{MESO}}}{\partial t}\right|_{\text {bgc }} & =\left.\frac{\partial Z_{\mathbb{N}, \mathbb{P}}}{\partial t}\right|_{\text {upt }}-\left.\frac{\partial Z_{\mathbb{N}, \mathbb{P}}}{\partial t}\right|_{\text {excr }} \\
& -\left.\frac{\partial Z_{\mathbb{N}, \mathbb{P}}}{\partial t}\right|_{\text {rel }}-\left.\frac{\partial Z_{\mathbb{N}, \mathbb{P}}}{\partial t}\right|_{\text {pred }}-\left.\frac{\partial Z_{\mathbb{N}, \mathbb{P}}}{\partial t}\right|_{\text {mort }} .
\end{aligned}
$$

The differences to the heterotrophic flagellates and microzooplankton are given by the release terms for stoichiometric adjustments for carbon, nitrogen, and phosphate (Eqs. 268 and 269) that replace nutrient release terms of the other two types (Eq. 36) and enhanced excretion for the scavenging on particulate matter $\stackrel{\text { MESO }}{q \text { Rexcr }}$ with respect to the uptake of living prey:

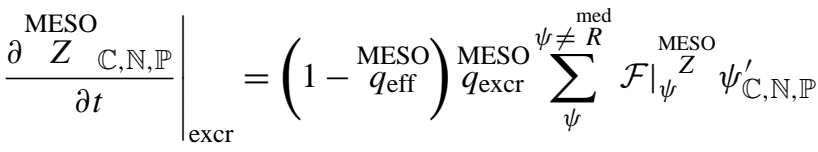

$$
\begin{aligned}
& +\left.q_{\text {Rexcr }}^{\text {MESO }} \mathcal{F}\right|_{\substack{\text { med } \\
R}} ^{\substack{\text { MESO } \\
Z}} \quad R^{\prime} R_{\mathbb{C}, \mathbb{N}, \mathbb{P}} .
\end{aligned}
$$

The hibernation formulation (optionally activated by the switch Z4_OW_SW) for over-wintering is triggered when the vertically integrated prey availability to mesozooplankton computed according to

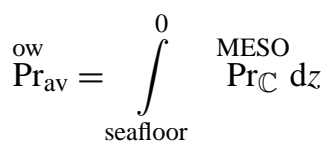

falls below the threshold $\stackrel{\text { ow }}{p}$ min.

In hibernation (overwintering) state the only active processes for mesozooplankton are respiration and mortality and using reduced the basal rates ( $r_{\text {owresp }}$ and $\left.r_{\text {owmort }}\right)$ with respect to the active state:

$$
\begin{aligned}
& \left.\frac{\partial Z_{\mathbb{C}}^{\mathrm{MESO}}}{\partial t}\right|_{\text {resp }}=r_{\text {owresp }} \stackrel{\text { MESO }}{Z_{\mathbb{C}}^{\prime}} \\
& \left.\frac{\partial^{\mathrm{MESO}} Z_{\mathbb{C}}}{\partial t}\right|_{\text {mort }}=r_{\text {owmort }} \begin{array}{c}
\text { MESO } \\
Z_{\mathbb{C}}^{\prime}
\end{array}
\end{aligned}
$$

\subsection{Heterotrophic bacteria}

Two alternative sub-modules for decomposition of organic material by bacteria are available in the ERSEM model involving different levels of decomposition of organic matter in the microbial food web.

\subsubsection{Original version}

In this version (Allen et al., 2002; Blackford et al., 2004; Baretta-Bekker et al., 1997) bacteria feed explicitly only on labile dissolved organic matter $R$. This is sufficient to create microbial loop dynamics in the model, opening the pathway from dissolved organic matter (DOM) over bacteria to zooplankton, while the other forms of substrate are recycled implicitly (see Eq. 70).

The biogeochemical dynamics of heterotrophic bacteria are here given by the equations:

$$
\begin{aligned}
\left.\frac{\partial B_{\mathbb{C}}}{\partial t}\right|_{\text {bgc }}= & \left.\frac{\partial B_{\mathbb{C}}}{\partial t}\right|_{\text {upt }}-\left.\frac{\partial B_{\mathbb{C}}}{\partial t}\right|_{\text {resp }} \\
& -\left.\frac{\partial B_{\mathbb{C}}}{\partial t}\right|_{\text {pred }}-\left.\frac{\partial B_{\mathbb{C}}}{\partial t}\right|_{\text {mort }}, \\
\left.\frac{\partial B_{\mathbb{N}, \mathbb{P}}}{\partial t}\right|_{\text {bgc }}= & \left.\frac{\partial B_{\mathbb{N}, \mathbb{P}}}{\text { partialt }}\right|_{\text {upt }}-\left.\frac{\partial B_{\mathbb{N}, \mathbb{P}}}{\partial t}\right|_{\text {rel }} \\
& -\left.\frac{\partial B_{\mathbb{N}, \mathbb{P}}}{\partial t}\right|_{\text {pred }}-\left.\frac{\partial B_{\mathbb{N}, \mathbb{P}}}{\partial t}\right|_{\text {mort }} .
\end{aligned}
$$

Bacterial uptake of DOM is given by a substrate massspecific turnover rate $r_{\text {lab }}^{B}$ for labile dissolved organic matter when substrate is scarce and by a maximum bacteria massspecific potential uptake regulated by temperature and limited by nutrient and oxygen conditions when substrate is abundant and the uptake per bacteria is saturated, regulated by the ratio of bacteria over substrate biomass:

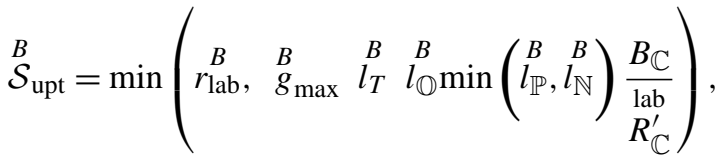

$$
\begin{aligned}
& \left.\frac{\partial B_{\mathbb{C}, \mathbb{N}, \mathbb{P}}}{\partial t}\right|_{\text {upt }}=\stackrel{B}{\mathcal{S}_{\text {upt }}} R^{\prime}{ }_{\mathbb{C}, \mathbb{N}, \mathbb{P}}^{\text {lab }},
\end{aligned}
$$

where $\stackrel{B}{\text { max }}_{\text {max }}$ is the maximum bacteria mass-specific uptake of bacteria.

Mortality is given as a constant fraction of bacteria biomass:

$\left.\frac{\partial B_{\mathbb{C}, \mathbb{N}, \mathbb{P}}}{\partial t}\right|_{\text {mort }}=\stackrel{B}{\text { mort }}_{\mathbb{C}, \mathbb{N}, \mathbb{P}}^{\prime}$,

where $\stackrel{B}{r}$ mort is a constant mass-specific mortality rate for bacteria. 

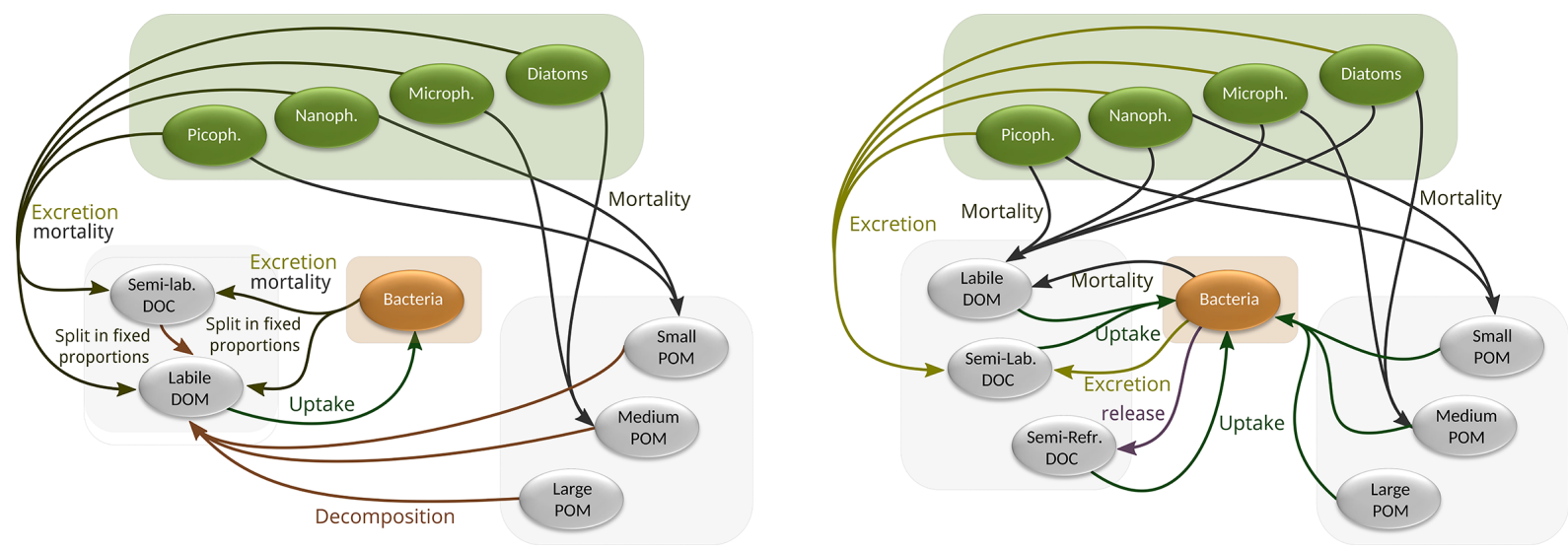

Figure 4. The microbial cycling of organic material for the standard bacteria model (left panel) and the dynamic decomposition model (right panel).

Bacteria respiration is computed according to activity respiration as an investment of activity in growth dependent on the oxygen state and a basal part:

$$
\begin{aligned}
\left.\frac{\partial B_{\mathbb{C}}}{\partial t}\right|_{\text {resp }}= & \left.\left(1-\stackrel{B}{q_{\text {high } \mathbb{O}}} \stackrel{B}{l_{\mathbb{O}}}-\stackrel{B}{q}_{\text {low } \mathbb{O}}\left(1-l_{\mathbb{O}}^{B}\right)\right) \frac{\partial B_{\mathbb{C}}}{\partial t}\right|_{\text {upt }} \\
& +\stackrel{B}{r}_{\text {resp }} \stackrel{B}{l}_{T}^{B} B_{\mathbb{C}}^{\prime}
\end{aligned}
$$

where $\stackrel{B}{r}_{\text {resp }}$ is the mass-specific basal respiration rate at rest (representing the maintenance cost of the metabolism in the absence of uptake activity) and $\stackrel{B}{q}_{\text {high } \mathbb{Q}, \text { low } \mathbb{O}}$ are the bacterial efficiencies at high and low oxygen levels.

Poor nutritional quality of the substrate may result in deprivation of nitrogen or phosphorus, resulting in nutrient uptake in competition with phytoplankton for external dissolved nutrient sources; otherwise, bacteria release superfluous nutrients into the environment. The internal stoichiometric quota of phosphorus is consequently balanced according to

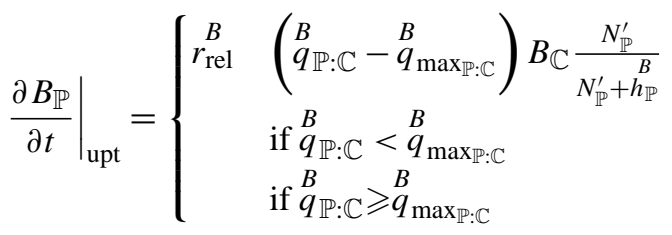

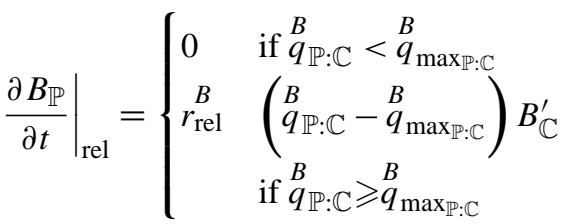

with $q_{\max : \mathbb{C}}$ being the optimal phosphorus to carbon quota of bacteria and $r_{\text {rel }}^{B}$ being the mass-specific release rate.
For nitrogen the internal stoichiometric quota is balanced using ammonium:

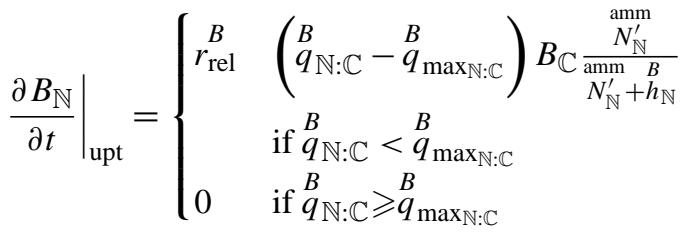

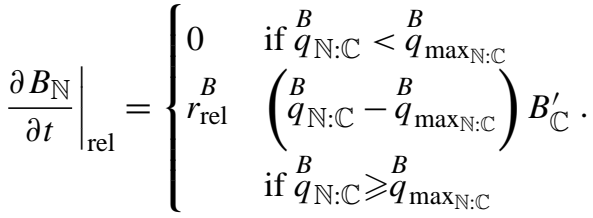

Predation on bacteria occurs only by heterotrophic flagellates and is given by

$$
\left.\frac{\partial B_{\mathbb{C}, \mathbb{P}, \mathbb{F}, \mathbb{N}}}{\partial t}\right|_{\text {pred }}=\left.\mathcal{F}\right|_{B} ^{\mathrm{HET}} B_{\mathbb{C}, \mathbb{P}, \mathbb{F}, \mathbb{N}}^{\prime}
$$

\subsubsection{Dynamic decomposition version}

In this version, activated with the DOCDYN preprocessing definition, the decomposition of particulate organic matter is directly mediated by bacteria, and the partition between labile dissolved organic matter and dissolved matter with longer degradation timescales (including the additional state of semi-refractory carbon) occurs in relation to the nutritional status of bacteria as opposed to the fixed parametric decomposition and partitioning of particles in the standard model. See also the following sections on the fluxes of particulate and dissolved organic matter (Sects. 3.5 and 3.4). The formulation includes the bacteria-mediated production of recalcitrant DOC (Hansell, 2013) and therefore provides the conceptual framework for an implementation of the microbial carbon pump (Jiao et al., 2014, 2010). However, the fractions of recalcitrant DOC with long turnover times $(\gg 1$ year) are not considered in the current formulation. The sub-model is 
an extended version of the formulation in Polimene et al. (2006, 2007).

The balance equations for bacteria here are mostly identical to the previous formulation (Eqs. 45 and 45) with the addition of the release of recalcitrant carbon:

$$
\begin{aligned}
\left.\frac{\partial B_{\mathbb{C}}}{\partial t}\right|_{\text {bgc }}= & \left.\frac{\partial B_{\mathbb{C}}}{\partial t}\right|_{\text {upt }}-\left.\frac{\partial B_{\mathbb{C}}}{\partial t}\right|_{\text {resp }}-\left.\frac{\partial B_{\mathbb{C}}}{\partial t}\right|_{\text {rel }} \\
& -\left.\frac{\partial B_{\mathbb{C}}}{\partial t}\right|_{\text {pred }}-\left.\frac{\partial B_{\mathbb{C}}}{\partial t}\right|_{\text {mort }}, \\
\left.\frac{\partial B_{\mathbb{N}, \mathbb{P}}}{\partial t}\right|_{\text {bgc }}= & \left.\frac{\partial B_{\mathbb{N}, \mathbb{P}}}{\partial t}\right|_{\text {upt }}-\left.\frac{\partial B_{\mathbb{N}, \mathbb{P}}}{\partial t}\right|_{\text {rel }} \\
& -\left.\frac{\partial B_{\mathbb{N}, \mathbb{P}}}{\partial t}\right|_{\text {pred }}-\left.\frac{\partial B_{\mathbb{N}, \mathbb{P}}}{\partial t}\right|_{\text {mort }}
\end{aligned}
$$

and an alternative formulation of uptake as in this formulation bacteria feed on all forms of particulate and dissolved organic matter:

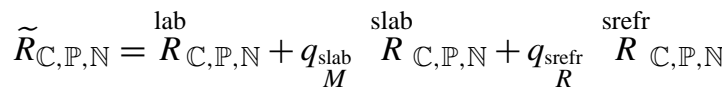

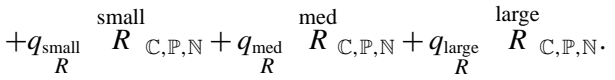

The parameters $q_{\psi}$ are non-dimensional turnover rates relative to $R$ turnover, leading to the following equations for substrate-specific and absolute uptake:

$$
\begin{aligned}
& \stackrel{B}{\mathcal{S}}_{\text {upt }}=\min \left(\begin{array}{lllll}
B & B & B & B_{\mathbb{C}} & B \\
g_{\max } & l_{T} & l_{\mathbb{O}} & \frac{B}{\widetilde{R}_{\mathbb{C}}}, & r_{\text {dis }}
\end{array}\right), \\
& \left.\frac{\partial B_{\mathbb{C}, \mathbb{N}, \mathbb{P}}}{\partial t}\right|_{\text {upt }}=\stackrel{B}{\mathcal{S}}_{\text {upt }} \widetilde{R}_{\mathbb{C}, \mathbb{P}, \mathbb{N}} .
\end{aligned}
$$

In this case carbon uptake is not nutrient limited as the internal stoichiometric quota of bacteria is balanced directly through the regulating fluxes releasing carbon into semilabile organic matter.

The release of recalcitrant carbon in the form of capsular semi-refractory material is assumed proportional by a factor of $q_{\text {srefr }}$ to the activity respiration representing the metabolic cost of the uptake activity:

$$
\begin{aligned}
& \left.\frac{\partial B_{\mathbb{C}}}{\partial t}\right|_{\text {rel }}=r_{\text {dis }}^{B} \max \left(0, \max \left(1-\frac{q_{\mathbb{P}: \mathbb{C}}}{q_{\max \mathbb{P}: \mathbb{C}}}, 1-\frac{q_{\mathbb{N}: \mathbb{C}}}{q_{\max _{\mathbb{N}: \mathbb{C}}}}\right)\right) B_{\mathbb{C}} \\
& +\left.q_{\text {srefr }}\left(1-\stackrel{B}{q}_{\text {high } \mathbb{O}} \stackrel{B}{l_{\mathbb{O}}}-\stackrel{B}{q}_{\text {low } \mathbb{O}}\left(1-l_{\mathbb{O}}^{B}\right)\right) \frac{\partial B_{\mathbb{C}}}{\partial t}\right|_{\text {growth }} .
\end{aligned}
$$

The bacteria-mediated fluxes of organic matter for the two different formulations of bacteria are illustrated in Fig. 4.

\subsection{Particulate organic matter}

The particulate matter $\stackrel{\chi}{R}: \chi=$ small, medium or large $)$ fluxes resulting from the above processes are composed of excre- tion and mortality inputs and decomposition and scavenging losses (for medium size particulate matter only) complemented by inputs resulting from mesozooplankton regulation of the internal stoichiometric ratio for large particulate matter. As the consumer types for simplicity do not include an internal component for iron or silicate, the corresponding component fluxes resulting from predation are directed to particulate matter as indirect excretion.

$$
\begin{aligned}
& \left.\frac{\partial \stackrel{\chi}{R}_{\mathbb{C}, \mathbb{N}, \mathbb{P}}}{\partial t}\right|_{\text {bgc }}=\left.\frac{\partial \stackrel{\chi}{R}_{\mathbb{C}, \mathbb{N}, \mathbb{P}}}{\partial t}\right|_{\text {excr }}+\left.\frac{\partial \stackrel{\chi}{R}_{\mathbb{C}, \mathbb{N}, \mathbb{P}}}{\partial t}\right|_{\text {mort }}-\left.\frac{\partial \stackrel{\chi}{R}_{\mathbb{C}, \mathbb{N}, \mathbb{P}}}{\partial t}\right|_{\text {decomp }} \\
& -\left.\frac{\partial R_{\mathbb{C}, \mathbb{N}, \mathbb{P}}^{\text {med }}}{\partial t}\right|_{\text {scav }}+\left.\frac{\partial \stackrel{\text { large }}{R} \mathbb{C}, \mathbb{N}, \mathbb{P}}{\partial t}\right|_{\text {rel }}, \\
& \left.\frac{\partial \stackrel{\chi}{R_{\mathbb{F}}}}{\partial t}\right|_{\text {bgc }}=\left.\frac{\partial \stackrel{\chi}{R_{\mathbb{F}}}}{\partial t}\right|_{\text {excr }}+\left.\frac{\partial \stackrel{\chi}{R_{\mathbb{F}}}}{\partial t}\right|_{\text {mort }}-\left.\frac{\partial \stackrel{\chi}{R}_{\mathbb{F}}}{\partial t}\right|_{\text {decomp }}\left[-\left.\frac{\partial \stackrel{\text { med }}{R}_{\mathbb{F}}}{\partial t}\right|_{\text {scav }}\right] . \\
& \left.\frac{\partial R_{\mathbb{S}}}{\partial t}\right|_{\text {bgc }}=\left.\frac{\partial R_{\mathbb{S}}}{\partial t}\right|_{\text {excr }}+\left.\frac{\partial R_{\mathbb{S}}}{\partial t}\right|_{\text {mort }} .
\end{aligned}
$$

Only the excretion by zooplankton (Eq. 34) results in particulate matter by a fraction of $1-\stackrel{\Psi}{q}_{\text {dloss }}$, while mortality of phytoplankton (Eqs. 13 and 20) and zooplankton (Eqs. 37 and 44$)$ both have a particulate component $\left(\stackrel{\psi}{\mathcal{Q}}_{\text {pmort }}\right.$ or $1-\stackrel{\Psi}{q}_{\text {dloss }}$ respectively):

$$
\begin{aligned}
& \left.\frac{\partial \stackrel{\chi}{R}_{\mathbb{C}}}{\partial t}\right|_{\text {excr }}=\left.\sum_{\Psi}\left(1-\stackrel{\Psi}{q}_{\text {dloss }}\right) \frac{\partial \stackrel{\Psi}{Z}_{\mathbb{C}}}{\partial t}\right|_{\text {excr }} \\
& \left.\frac{\partial \stackrel{\chi}{R}_{\mathbb{N}, \mathbb{P}}}{\partial t}\right|_{\text {excr }}=\left.\sum_{\Psi}\left(1-\stackrel{\text { lab }}{p}_{\text {cyto }_{\mathbb{N}, \mathbb{P}}} \stackrel{\Psi}{q}_{\text {dloss }}\right) \frac{\partial Z_{\mathbb{N}, \mathbb{P}}}{\partial t}\right|_{\text {excr }}
\end{aligned}
$$

$$
\begin{aligned}
\left.\frac{\partial \stackrel{\chi}{R}_{\mathbb{C}, \mathbb{N}, \mathbb{P}, \mathbb{F}, \mathbb{S}}}{\partial t}\right|_{\text {mort }} & =\left.\sum_{\psi} \stackrel{\psi}{\mathcal{Q}_{\text {pmort }}} \frac{\partial \stackrel{\psi}{P}_{\mathbb{C}, \mathbb{N}, \mathbb{P}, \mathbb{F}, \mathbb{S}}}{\partial t}\right|_{\text {mort }} \\
& +\left.\sum_{\Psi}\left(1-\stackrel{\Psi}{q}{ }_{\text {dloss }}\right) \frac{\partial \stackrel{\Psi}{Z}_{\mathbb{C}, \mathbb{N}, \mathbb{P}}}{\partial t}\right|_{\text {mort }},
\end{aligned}
$$

where $\stackrel{\text { lab }}{p}_{\text {cyto }_{\mathbb{N}, \mathbb{P}}}$ reflects the relative nitrogen or phosphorus content of cytoplasm with respect to the structural components assuming that the dissolved losses of zooplankton through excretion are largely of cytoplasm origin and $\stackrel{\Psi}{q}$ dloss is the dissolved fraction of zooplankton losses. The partition of phytoplankton lysis for each functional type is given as 
Table 2. Particulate organic matter and its origin.

\begin{tabular}{|c|c|}
\hline POM type & Originating from \\
\hline Small particulate organic matter $(\stackrel{\text { small }}{R})$ & Nano- and pico-phytoplankton $(\stackrel{\text { nano pico }}{P}, \stackrel{\text { HET }}{P})$, heterotrophic flagellates $(\stackrel{Z}{Z})$ \\
\hline $\begin{array}{l}\text { Medium-size particulate organic matter }(\stackrel{\text { med }}{R}) \\
\text { Large particulate organic matter }(\stackrel{R}{R})\end{array}$ & $\begin{array}{l}\text { Microphytoplankton and diatoms }(\stackrel{\text { micro dia }}{P}, P) \text {, microzooplankton }(\stackrel{\text { MICRO }}{Z}) \\
\text { Mesozooplankton }\left(\begin{array}{c}\text { MESO } \\
\text { M }\end{array}\right)\end{array}$ \\
\hline
\end{tabular}

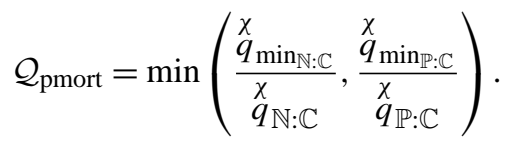

The size classes of particulate organic matter $\chi$ in these equations originate from the phytoplankton types $\stackrel{\psi}{P}$ and zooplankton types $\stackrel{\Psi}{Z}$ as given in Table 2.

Scavenging of mesozooplankton on medium size particulate organic matter results from Eq. (31):

$$
\left.\frac{\partial R \mathbb{C}, \mathbb{N}, \mathbb{P}, \mathbb{F}, \mathbb{S}}{\partial t}\right|_{\text {scav }}=\left.\mathcal{F}\right|_{\substack{\text { med } \\ R}} ^{\substack{\text { MESO } \\ \text { med }}} R_{\mathbb{C}, \mathbb{N}, \mathbb{P}, \mathbb{F}, \mathbb{S}}^{\prime} .
$$

Additional large particulate organic matter may result from the mesozooplankton release flux $\left.\frac{\partial R \mathbb{C}}{\partial t}\right|_{\text {rel }}=\left.\frac{\partial Z_{\mathbb{C}}}{\partial t}\right|_{\text {rel }}$ (Eq. 268).

The decomposition of particulate matter is dependent on the bacteria sub-model applied. In the case of the standard bacteria model (Sect. 3.3.1) it is converted to dissolved organic matter proportionally to the amount of substrate available by the rate $\stackrel{x}{r}$ decomp and modified by the nutritional status of the substrate in relation to the Redfield ratio $q_{\text {ref }_{\mathbb{C}: \mathbb{N}}}$ :

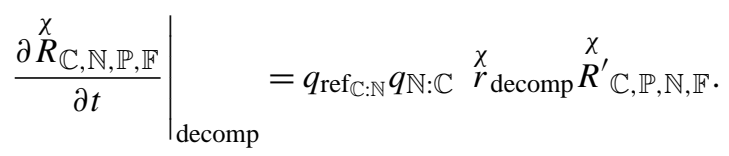

For the model with dynamic decomposition (Sect. 3.3.2) directly mediated by bacteria, the decomposition fluxes are given by the bacterial uptake resulting from Eqs. (58), (59), and (60) as

$$
\left.\frac{\partial \stackrel{\chi}{R}_{\mathbb{C}, \mathbb{N}, \mathbb{P}, \mathbb{F}}}{\partial t}\right|_{\text {decomp }}=-\stackrel{B}{\mathcal{S}}_{\text {growth }} r_{M}^{\underset{M}{R^{\prime}}} \stackrel{\chi}{\mathbb{C}, \mathbb{P}, \mathbb{N}, \mathbb{F}} .
$$

The iron and silicate component of phytoplankton taken up by zooplankton in Eqs. (21) and (25) are for simplicity directly converted to particulate matter:

$$
\begin{aligned}
& \left.\frac{\partial \stackrel{\chi}{R_{\mathbb{F}}}}{\partial t}\right|_{\text {excr }}=\left.\sum_{\psi, \Psi} \mathcal{F}\right|_{P} ^{\stackrel{\Psi}{Z}}{\stackrel{\psi^{\prime}}{P}}_{\mathbb{F}}
\end{aligned}
$$

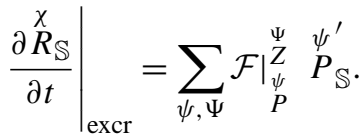

In the case of silicate the particulate organic matter types are determined by the predator that ingested the prey and directly releases the silicate contained in the frustule. They are consequently distributed analogous to the zooplankton excretion:

- small particulate organic matter $(\stackrel{\text { small }}{R})$ : heterotrophic flagellates $(\stackrel{\text { HET }}{Z})$,

- medium size particulate organic matter $\stackrel{\text { med }}{R})$ : microzooplankton $(\stackrel{\text { MICRO }}{Z})$,

- large particulate organic matter $(\stackrel{\text { large }}{R})$ : mesozooplankton $(\stackrel{Z}{Z})$

For iron, on the contrary, the size of particulate iron is given by the prey size class and taken analogous to phytoplankton lysis reflecting the assimilation of iron into the cytoplasm:

- small particulate organic matter $(\stackrel{\text { small }}{R})$ : nano- and picophytoplankton $(\stackrel{\text { nano pico }}{P}, \stackrel{P}{P})$,

- medium size particulate $\operatorname{organic}$ matter $(\stackrel{\text { med }}{R})$ : microphy-

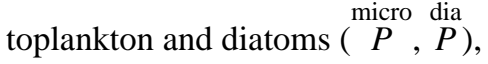

- large particulate organic matter $(\stackrel{\text { large }}{R})$ : none.

\subsection{Dissolved organic matter}

The partition of labile dissolved, semi-labile, and semirefractory carbon originating from bacteria substantially differs between the standard bacteria model (Sect. 3.3.1) and the bacteria model with dynamic decomposition (Sect. 3.3.2). 
For the standard bacteria model the fluxes of dissolved organic matter are affected by uptake, excretion, mortality, decomposition, and remineralization:

$$
\begin{aligned}
& \left.\frac{\partial R_{\mathbb{C}, \mathbb{N}, \mathbb{P}}^{\text {lab }}}{\partial t}\right|_{\text {bgc }}=\left.\frac{\partial R_{\mathbb{C}, \mathbb{N}, \mathbb{P}}^{\text {lab }}}{\partial t}\right|_{\text {excr }}+\left.\frac{\partial R_{\mathbb{C}, \mathbb{N}, \mathbb{P}}}{\partial t}\right|_{\text {mort }}+\left.\frac{\partial R_{\mathbb{C}, \mathbb{N}, \mathbb{P}}^{\text {lab }}}{\partial t}\right|_{\text {decomp }} \\
& -\left.\frac{\partial R_{\mathbb{C}, \mathbb{N}, \mathbb{P}}}{\partial t}\right|_{\text {upt }}\left[-\left.\frac{\partial R_{\mathbb{N}, \mathbb{P}}}{\partial t}\right|_{\text {remin }}\right], \\
& \left.\frac{\partial R_{\mathbb{C}}}{\partial t}\right|_{\text {bgc }} ^{\text {slab }}=\left.\frac{\partial R_{\mathbb{C}}}{\partial t}\right|_{\text {excr }} ^{\text {slab }}+\left.\frac{\partial R_{\mathbb{C}}}{\partial t}\right|_{\text {mort }} ^{\text {slab }}-\left.\frac{\partial R_{\mathbb{C}}}{\partial t}\right|_{\text {decomp }}
\end{aligned}
$$

The losses of bacteria, phytoplankton and zooplankton in dissolved carbon are fractionated at a constant quota $q_{\text {dis }}$ in between labile and semi-labile DOC. Excretion towards the dissolved forms of organic matter may originate from phytoplankton (Eq. 11), or zooplankton (Eq. 34):

$$
\begin{aligned}
& \left.\frac{\partial R_{\mathbb{C}}}{\partial t}\right|_{\text {excr }}=q_{\text {dis }}\left(\left.\sum_{\psi} \frac{\partial P_{\mathbb{C}}}{\partial t}\right|_{\text {excr }}+\left.\sum_{\Psi} \stackrel{\Psi}{q}_{\text {dloss }} \frac{\partial Z_{\mathbb{C}}}{\partial t}\right|_{\text {excr }}\right) \\
& \left.\frac{\partial R_{\mathbb{C}}^{\text {slab }}}{\partial t}\right|_{\text {excr }}=\left(1-q_{\text {dis }}\right)\left(\left.\sum_{\psi} \frac{\partial P_{\mathbb{C}}^{\psi}}{\partial t}\right|_{\text {excr }}+\left.\sum_{\Psi} \stackrel{\Psi}{q}_{\text {dloss }} \frac{\partial Z_{\mathbb{C}}}{\partial t}\right|_{\text {excr }}\right),
\end{aligned}
$$

where $\stackrel{\Psi}{q}$ dloss is the dissolved fraction of the zooplankton losses.

Mortality input may originate from all three trophic levels (Eqs. 49, 13, 37, 43):

$$
\begin{aligned}
\left.\frac{\partial R_{\mathbb{C}}^{\text {lab }}}{\partial t}\right|_{\text {mort }}= & q_{\text {lab }}\left(\left.\frac{\partial B_{\mathbb{C}}}{\partial t}\right|_{\text {mort }}+\left.\sum_{\psi}\left(1-\stackrel{\psi}{\mathcal{Q}}_{\text {pmort }}\right) \frac{\partial \stackrel{\psi}{P}_{\mathbb{C}}}{\partial t}\right|_{\text {mort }}\right. \\
& \left.+\left.\sum_{\Psi} \stackrel{\Psi}{q}_{\text {dloss }} \frac{\partial \stackrel{\Psi}{Z}_{\mathbb{C}}}{\partial t}\right|_{\text {mort }}\right)
\end{aligned}
$$

$$
\begin{aligned}
\left.\frac{\partial R_{\mathbb{C}}^{\text {slab }}}{\partial t}\right|_{\text {mort }} & =\left(1-q_{\text {lab }}\right)\left(\left.\frac{\partial B_{\mathbb{C}}}{\partial t}\right|_{\text {mort }}+\left.\sum_{\psi}(1-\stackrel{\mathcal{Q}}{\text { pmort }}) \frac{\partial P_{\mathbb{C}}}{\partial t}\right|_{\text {mort }}\right. \\
& \left.+\left.\sum_{\Psi} \stackrel{\Psi}{q}_{\text {dloss }} \frac{\partial \stackrel{\Psi}{Z}_{\mathbb{C}}}{\partial t}\right|_{\text {mort }}\right) .
\end{aligned}
$$

In addition, the decomposition of the particulate matter types $\stackrel{\Psi}{(R}: \Psi=$ small, medium, or large, Eq. 70$)$ and of semilabile dissolved organic carbon $R_{\mathbb{C}}$ is directly converted to labile dissolved organic matter $(R)$ according to $\left.\frac{\partial R_{\mathbb{C}, \mathbb{N}, \mathbb{P}}^{\text {lab }}}{\partial t}\right|_{\text {decomp }}=\left.\sum_{\Psi} \frac{\partial R_{\mathbb{C}, \mathbb{N}, \mathbb{P}}}{\partial t}\right|_{\text {decomp }}+\left.\frac{\partial R_{\mathbb{C}}^{\text {slab }}}{\partial t}\right|_{\text {decomp }}$

$\left.\frac{\partial R_{\mathbb{C}}}{\partial t}\right|_{\text {decomp }}=r_{\text {decomp }}^{\text {slab }} R_{\mathbb{C}}^{\text {slab }}$

without explicit mediation of bacteria.

In the dynamic decomposition model the fluxes of dissolved organic matter are a result of uptake, excretion, mortality and remineralization:

$$
\begin{aligned}
& \left.\frac{\partial R_{\mathbb{C}, \mathbb{N}, \mathbb{P}}}{\partial t}\right|_{\text {bgc }}=\left.\frac{\partial R_{\mathbb{C}, \mathbb{N}, \mathbb{P}}}{\partial t}\right|_{\text {excr }}+\left.\frac{\partial R_{\mathbb{C}, \mathbb{N}, \mathbb{P}}}{\partial t}\right|_{\text {mort }} \\
& -\left.\frac{\partial R_{\mathbb{C}, \mathbb{N}, \mathbb{P}}}{\partial t}\right|_{\text {upt }}\left[-\left.\frac{\partial R_{\mathbb{N}, \mathbb{P}}^{\text {lab }}}{\partial t}\right|_{\text {remin }}\right], \\
& \left.\frac{\partial R_{\mathbb{C}}^{\text {slab }}}{\partial t}\right|_{\text {bgc }}=\left.\frac{\partial R_{\mathbb{C}}^{\text {slab }}}{\partial t}\right|_{\text {excr }}+\left.\frac{\partial R_{\mathbb{C}}^{\text {slab }}}{\partial t}\right|_{\text {mort }}-\left.\frac{\partial R_{\mathbb{C}}}{\partial t}\right|_{\text {upt }}, \\
& \left.\frac{\partial \stackrel{\text { srefr }}{R} \mathbb{C}}{\partial t}\right|_{\text {bgc }}=\left.\frac{\partial \stackrel{\text { srefr }}{R} \mathbb{C}}{\partial t}\right|_{\text {excr }}-\left.\frac{\partial \stackrel{\text { srefr }}{R} \mathbb{C}}{\partial t}\right|_{\text {upt }} \text {. }
\end{aligned}
$$

Here, the fractionation of dissolved organic matter originating from bacteria and phytoplankton is based on the originating process. This reflects the capacity of bacteria to utilize different forms of substrate with lysis/mortality contributing to the labile DOM pool, while excretion of carbon occurs in semi-labile form, and discarding the less digestible forms adding semi-refractory organic matter to the set of state variables. Zooplankton losses are treated identically with respect to the standard bacteria model.

Excretion of DOC may originate from the phyto- and zooplankton excretion (Eqs. 11 and 34), the regulation of the bacterial stoichiometric quota (Eq. 61), and excess bacterial growth:

$$
\left.\frac{\partial R_{\mathbb{C}}^{\text {lab }}}{\partial t}\right|_{\text {excr }}=\left.\sum_{\Psi} q_{\mathrm{dis}} \stackrel{\Psi}{q_{\mathrm{dloss}}} \frac{\partial \stackrel{\Psi}{Z_{\mathbb{C}}}}{\partial t}\right|_{\text {excr }},
$$




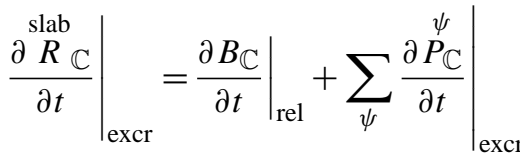

$$
\begin{aligned}
& +\left.\sum_{\Psi}\left(1-q_{\mathrm{dis}}\right) \stackrel{\Psi}{q} \mathrm{dloss} \frac{\partial \stackrel{\Psi}{Z}_{\mathbb{C}}}{\partial t}\right|_{\text {excr }}, \\
& \left.\frac{\partial \stackrel{\text { srefr }}{R} \mathbb{C}}{\partial t}\right|_{\text {excr }}=p_{\text {srefr }}\left(1-\stackrel{B}{\text { high } \mathbb{O}}^{l_{\mathbb{O}}}\right. \\
& \left.-\stackrel{B}{q_{\text {low } \mathbb{O}}}\left(1-r_{\mathbb{O}}^{B}\right)\right)\left.\frac{\partial B_{\mathbb{C}}}{\partial t}\right|_{\text {growth }},
\end{aligned}
$$

while the non-particulate part of mortality/lysis is split according to

$$
\begin{aligned}
\left.\frac{\partial R_{\mathbb{C}}^{\text {lab }}}{\partial t}\right|_{\text {mort }}= & \left.\frac{\partial B_{\mathbb{C}}}{\partial t}\right|_{\text {mort }}+\left.\sum_{\psi}\left(1-\stackrel{\psi}{\mathcal{Q}_{\text {pmort }}}\right) \frac{\partial P_{\mathbb{C}}^{\psi}}{\partial t}\right|_{\text {lysis }} \\
& +\sum_{\Psi} q_{\text {dis }} \stackrel{\Psi}{q} \text { dloss }\left._{\frac{\Psi}{Z_{\mathbb{C}}}}\right|_{\text {mort }} \\
\left.\frac{\partial{ }_{\text {slab }}^{R}}{\partial t}\right|_{\text {mort }}= & \left.\sum_{\Psi}\left(1-q_{\text {dis }}\right) \stackrel{\Psi}{q_{\text {dloss }}} \frac{\partial \underset{\Psi}{Z_{\mathbb{C}}}}{\partial t}\right|_{\text {mort }} .
\end{aligned}
$$

Uptake of labile dissolved matter by bacteria is given by

$\left.\frac{\partial R_{\mathbb{C}, \mathbb{N}, \mathbb{P}}}{\partial t}\right|_{\text {upt }}=\stackrel{B}{\mathcal{S}}_{\text {growth }}{\stackrel{\text { lab }}{R^{\prime}} \mathbb{C}, \mathbb{N}, \mathbb{P},}$

where the substrate mass-specific uptake of bacteria $\mathcal{S}_{\text {growth }}$ is given in Eq. (47) for the standard decomposition model and in Eq. (59) for the dynamic decomposition model.

The remaining terms are identical for both decomposition sub-models. Excretion and mortality of nitrogen and phosphorus result in the dissolved fluxes

$$
\begin{aligned}
& \left.\frac{\partial \stackrel{1 a b}{R}_{\mathbb{N}, \mathbb{P}}}{\partial t}\right|_{\text {excr }}=\left.\sum_{\Psi} \stackrel{\Psi}{q}_{\text {dloss }} \stackrel{\text { lab }}{p}_{\text {cyto }_{\mathbb{N}, \mathbb{P}}} \frac{\partial \stackrel{\Psi}{Z}_{\mathbb{N}, \mathbb{P}}}{\partial t}\right|_{\text {excr }} \\
& \left.\frac{\partial \stackrel{l a b}{R}_{\mathbb{N}, \mathbb{P}}}{\partial t}\right|_{\text {mort }}=\left.\frac{\partial B_{\mathbb{N}, \mathbb{P}}}{\partial t}\right|_{\text {mort }}+\left.\sum_{\psi}\left(1-\stackrel{\psi}{\mathcal{Q}}_{\text {pmort }}\right) \frac{\partial \stackrel{\psi}{P}_{\mathbb{N}, \mathbb{P}}}{\partial t}\right|_{\text {mort }} \\
& +\left.\sum_{\Psi} \stackrel{\Psi}{q}_{\mathrm{dloss}} \frac{\partial \stackrel{\Psi}{Z}_{\mathbb{N}, \mathbb{P}}}{\partial t}\right|_{\text {mort }}
\end{aligned}
$$

Remineralization of dissolved organic nutrients into inorganic form is given by fixed mass-specific remineralization rates $r_{\mathrm{rem}_{N, \mathbb{P}}}$ : $\left.\frac{\partial R_{\mathbb{N}, \mathbb{P}}^{\mathrm{lab}}}{\partial t}\right|_{\text {remin }}=r_{\mathrm{rem}_{\mathbb{N}, \mathbb{P}}} \stackrel{\text { dis }}{R^{\prime}} \mathbb{N}, \mathbb{P}$

\subsection{Calcification}

The model in its current form does not include calcifiers as a dedicated functional group given the limited knowledge of the physiological constraint of calcification. Therefore, the process of calcification is not directly modelled, but is treated implicitly by considering part of the nanophytoplankton to act as calcifiers. Calcification processes are inferred from the system dynamics based on the assumption of a given ratio between particulate inorganic carbon over particulate organic carbon in sedimenting material, usually referred to as the rain ratio. Here this ratio is used as a proxy for the calcite production matching the local increase of POC originating from nanophytoplankton. Since the rain ratio has been defined for the sinking fluxes and calcite is the more resistant mineral, we limit the description to calcite in this part of the model, neglecting aragonite. This approach is similar to the implementations in other biogeochemical models, e.g. PISCES (Gehlen et al., 2007) or MEDUSA (Yool et al., 2013).

In this context the local rain ratio is based on a reference ratio $q_{\text {rain } 0}$ that varies according to the regulating factors calc calc calc $l_{\mathbb{C}}, l_{T}$, and $l_{\langle\mathbb{N P}\rangle}$ given in Eq. (260) or Eqs. (262), (264), and (265):

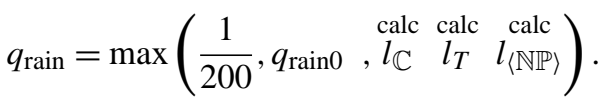

The calcite dynamics are then described by the equation

$\left.\frac{\partial L_{\mathbb{C}}^{\text {calc }}}{\partial t}\right|_{\text {bgc }}=\left.\frac{\partial L_{\mathbb{C}}}{\partial t}\right|_{\text {mort }}+\left.\frac{\partial L_{\mathbb{C}}}{\partial t}\right|_{\text {pred }}+\left.\frac{\partial L_{\mathbb{C}}^{\text {calc }}}{\partial t}\right|_{\text {sed }}-\left.\frac{\partial L_{\mathbb{C}}}{\partial t}\right|_{\text {dis }}$.

The contribution of nanophytoplankton lysis to calcite production is proportional to the particulate fraction of lysis (compare Eq. 67) by the rain ratio

$\left.\frac{\partial L_{\mathbb{C}}^{\text {calc }}}{\partial t}\right|_{\text {mort }}=\left.q_{\text {rain }} \mathcal{Q}_{\text {pmort }}^{\text {nano }} \frac{\partial P_{\mathbb{C}}}{\partial t}\right|_{\text {mort }}$.

Ingestion of nanophytoplankton and subsequent dissolution in zooplankton guts contributes with a fraction $q_{\text {gutdiss }}$ of the excreted part of nanophytoplankton uptake by the various zooplankton groups (compare Eqs. 14 and 34):

$\left.\frac{\partial L_{\mathbb{C}}^{\text {calc }}}{\partial t}\right|_{\text {pred }}=\left.q_{\text {rain }} q_{\text {gutdiss }}\left(1-\stackrel{\chi}{q}_{\text {eff }}\right) \stackrel{\chi}{q}_{\text {excr }}^{\chi} \sum_{\Psi} \mathcal{F}\right|_{P} ^{\stackrel{\Psi}{Z}} \underset{P}{i n o} P_{\mathbb{C}}^{\prime}$. 
As sedimentation of nanophytoplankton contributes to the organic carbon considered in the rain ratio, the matching contribution to calcite production is computed as

$$
\left.\frac{\partial L_{\mathbb{C}}^{\text {calc }}}{\partial t}\right|_{\text {sed }}=\left.q_{\text {rain }} \frac{\partial P P_{\mathbb{C}}^{\text {nano }}}{\partial t}\right|_{\text {sed }}
$$

with the sinking rate $\left.\frac{\partial P P_{\mathbb{C}}}{\partial t}\right|_{\text {sed }}$ given in Eq. (143).

Dissolution of calcite is proportional to the current concentration of calcite with a maximum rate of $r_{\mathrm{dis}}$, regulated by $\stackrel{\text { calc }}{l_{\mathbb{C}}}($ Eqs. 261 or 263$)$ :

$\left.\frac{\partial L_{\mathbb{C}}}{\partial t}\right|_{\text {dis }}=r_{\text {dis }} \quad$\begin{tabular}{lll}
$l_{\mathbb{C}}$ & $L_{\mathbb{C}}^{\prime}$ \\
\hline
\end{tabular}

Note that while the calcification rates are implicitly derived from the rain ratio and are not directly modelled processes, this formulation is still conservative as all sources and sinks of calcite are balanced by dissolved inorganic carbon (DIC; see Eqs. 120 and 121).

The solution of the calcite dynamics is optional and activated by the CALC preprocessing switch.

\subsection{Inorganic components}

The dynamics of dissolved inorganic nutrients in the model are given by uptake of phytoplankton and bacteria and are resupplied locally by remineralization and excretion. Dissolved inorganic iron is additionally subject to scavenging.

$$
\begin{aligned}
& \left.\frac{\partial N_{\mathbb{N}}^{\text {ox }}}{\partial t}\right|_{\text {bgc }}=\left.\frac{\partial N_{\mathbb{N}}}{\partial t}\right|_{\text {nitr }}-\left.\frac{\partial N_{\mathbb{N}}}{\partial t}\right|_{\text {upt }}, \\
& \left.\frac{\partial N_{\mathbb{N}}}{\partial t}\right|_{\text {bgc }}=\left.\frac{\partial N_{\mathbb{N}}}{\partial t}\right|_{\text {remin }}+\left.\frac{\partial N_{\mathbb{N}}}{\partial t}\right|_{\text {rel }}-\left.\frac{\partial N_{\mathbb{N}}}{\partial t}\right|_{\text {upt }}-\left.\frac{\partial N_{\mathbb{N}}}{\partial t}\right|_{\text {nitr }}, \\
& \left.\frac{\partial N_{\mathbb{P}}}{\partial t}\right|_{\text {bgc }}=\left.\frac{\partial N_{\mathbb{P}}}{\partial t}\right|_{\text {remin }}+\left.\frac{\partial N_{\mathbb{P}}}{\partial t}\right|_{\text {rel }}-\left.\frac{\partial N_{\mathbb{P}}}{\partial t}\right|_{\text {upt }}, \\
& \left.\frac{\partial N_{\mathbb{S}}}{\partial t}\right|_{\text {bgc }}=\left.\frac{\partial N_{\mathbb{S}}}{\partial t}\right|_{\text {rel }}-\left.\frac{\partial N_{\mathbb{S}}}{\partial t}\right|_{\text {upt }}, \\
& \left.\frac{\partial N_{\mathbb{F}}}{\partial t}\right|_{\text {bgc }}=\left.\frac{\partial N_{\mathbb{F}}}{\partial t}\right|_{\text {remin }}+\left.\frac{\partial N_{\mathbb{F}}}{\partial t}\right|_{\text {rel }}-\left.\frac{\partial N_{\mathbb{F}}}{\partial t}\right|_{\text {upt }}-\left.\frac{\partial N_{\mathbb{F}}}{\partial t}\right|_{\text {scav }} .
\end{aligned}
$$

Oxidized nitrogen in the water column is taken up only by the four phytoplankton types $\stackrel{\psi}{P}$ following Eq. (15) according to external availability

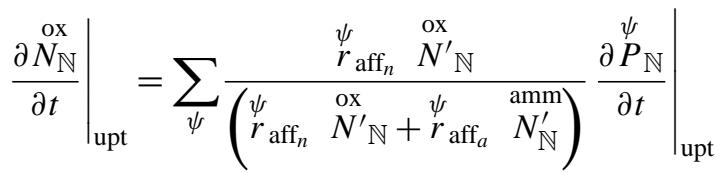

and regenerated exclusively by nitrification

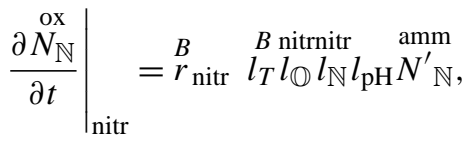

where $\stackrel{B}{r}$ nitr is the maximum ammonium mass-specific nitrification rate at reference temperature. In the absence of explicit nitrifiers, nitrification is modelled as an implicit process depending on multiple environmental factors, based on temperature, oxygen, and available ammonium taking into account the poor competitiveness of nitrifying microbes with respect to other pelagic consumers of ammonium (Ward, 2008). The various regulation and limitation factors $l_{T}, l_{\mathbb{O}}, l_{\mathbb{N}}$, and $l_{\mathrm{pH}}$ are given in Sect. 6.1.

Ammonium is taken up by phytoplankton as the reduced part of total nitrogen uptake (Eq. 15) and bacteria when nitrogen limited,

$$
\begin{aligned}
\left.\frac{\partial N_{\mathbb{N}}^{\mathrm{amm}}}{\partial t}\right|_{\text {upt }} & =\sum_{\psi} \frac{\psi_{\mathrm{aff}_{n}}}{N^{\prime}} \mathbb{N}_{\mathbb{N}} \\
& +\left.\frac{\partial B_{\mathbb{N}}}{\partial t}\right|_{\text {upt }},
\end{aligned}
$$

and remineralized according to Eq. (93):

$\left.\frac{\partial N_{\mathbb{N}}^{\mathrm{amm}}}{\partial t}\right|_{\text {remin }}=r_{\text {rem }_{\mathbb{N}}} \stackrel{\text { lab }}{\prime}_{\mathbb{N}}^{\prime}$.

Ammonium is released by the phytoplankton types $\psi$ (Eq. 15) when respiration exceeds photosynthesis or when above their luxury storage capacity and by the zooplankton types $\Psi$ (Eqs. 36 and 269) and bacteria (Eq. 54) when above their optimal quota

$\left.\frac{\partial N_{\mathbb{N}}^{\mathrm{amm}}}{\partial t}\right|_{\text {rel }}=\left.\sum_{\psi} \frac{\partial \stackrel{\psi}{P}_{\mathbb{N}}}{\partial t}\right|_{\text {rel }}+\left.\sum_{\Psi} \frac{\partial \stackrel{\Psi}{Z}_{\mathbb{N}}}{\partial t}\right|_{\text {rel }}+\left.\frac{\partial B_{\mathbb{N}}}{\partial t}\right|_{\text {rel }}$.

Ammonium concentrations may be further reduced by nitrification:

$\left.\frac{\partial \stackrel{\mathrm{amm}}{N_{\mathbb{N}}}}{\partial t}\right|_{\text {nitr }}=\stackrel{B}{r_{\text {nitr }}} \stackrel{B}{l_{T}} l_{\mathbb{O}_{\text {nitr }}} l_{\mathbb{N}_{\text {nitr }}} l_{\mathrm{pH}}{ }^{\mathrm{amm}} N_{\mathbb{N}}^{\prime}$

Phosphorus dynamics are analogous to nitrogen dynamics but simplified with only one dissolved inorganic pool being 
considered in the model. It is taken up according to Eqs. (15) and (52)

$$
\left.\frac{\partial N_{\mathbb{P}}}{\partial t}\right|_{\text {upt }}=\left.\sum_{\psi} \frac{\partial \stackrel{\psi}{P_{\mathbb{P}}}}{\partial t}\right|_{\text {upt }}+\left.\frac{\partial B_{\mathbb{P}}}{\partial t}\right|_{\text {upt }},
$$

released following Eqs. (15), (52), (36), and (37)

$$
\left.\frac{\partial N_{\mathbb{P}}}{\partial t}\right|_{\text {rel }}=\left.\sum_{\Psi} \frac{\partial \stackrel{\Psi}{Z}_{\mathbb{P}}}{\partial t}\right|_{\text {rel }}-\left.\sum_{\psi} \frac{\partial \stackrel{\psi}{P}_{\mathbb{P}}}{\partial t}\right|_{\text {upt }}+\left.\frac{\partial B_{\mathbb{P}}}{\partial t}\right|_{\text {rel }},
$$

and remineralized as given in Eq. (54):

$$
\left.\frac{\partial N_{\mathbb{P}}}{\partial t}\right|_{\text {remin }}=r_{\text {rem } \mathbb{P}} \stackrel{\text { lab }}{R^{\prime}} \text {. }
$$

Iron is taken up only by phytoplankton (Eq. 15)

$$
\left.\frac{\partial N_{\mathbb{F}}}{\partial t}\right|_{\text {upt }}=\left.\sum_{\psi} \frac{\partial \stackrel{\psi}{P}_{\mathbb{F}}}{\partial t}\right|_{\text {upt }}
$$

and subject to scavenging due to hydroxide, treated similarly as in Aumont et al. (2003) and Vichi et al. (2007):

$$
\left.\frac{\partial N_{\mathbb{F}}}{\partial t}\right|_{\text {scav }}=r_{\mathbb{F s c a v}} \max \left(0, N_{\mathbb{F}}^{\prime}\right) \text {, }
$$

where $r_{\mathbb{F s c a v}}$ is a threshold concentration over which scavenging occurs, here fixed at $0.6 \mu \mathrm{mol} \mathrm{m} \mathrm{m}^{-3}$.

Iron is released by phytoplankton (Eq. 15)

$$
\left.\frac{\partial N_{\mathbb{F}}}{\partial t}\right|_{\text {rel }}=\left.\sum_{\psi} \frac{\partial \stackrel{\psi}{P}_{\mathbb{F}}}{\partial t}\right|_{\text {rel }}
$$

and implicitly remineralized by mesozooplankton scavenging of particulate organic matter (Eq. 69) and bacterial consumption of particulate matter (Eqs. 70 and 71)

$$
\left.\frac{\partial N_{\mathbb{F}}}{\partial t}\right|_{\text {remin }}=\left.\mathcal{F}\right|_{\substack{\text { med } \\ R}} ^{\substack{\text { MESO } \\ \text { med }}} R_{\mathbb{F}}^{\prime}+\left.\frac{\partial R_{\mathbb{F}}^{\chi}}{\partial t}\right|_{\text {decomp }} .
$$

It is assumed here that the feeding activity of scavenging zooplankton increases the bio-availability and accelerates the decomposition of particulate iron.

Silicate is taken up,

$$
\left.\frac{\partial N_{\mathbb{S}}}{\partial t}\right|_{\text {uptake }}=\stackrel{\text { dia }}{q} \text { ref } \mathbb{S}: \mathbb{C}_{\text {dia }}^{\text {dia }} \text { growth },
$$

and released,

$$
\left.\frac{\partial N_{\mathbb{S}}}{\partial t}\right|_{\text {rel }}=\stackrel{\text { dia }}{P_{\mathbb{S}}^{\prime}}-\stackrel{\text { dia }}{q}_{\text {ref }: \mathbb{C}} \stackrel{\text { dia }}{P_{\mathbb{C}}^{\prime}}
$$

exclusively by diatoms (Eq. 22). It is not remineralized in the pelagic part of the system.

This neglect of silicate conversion into inorganic form in the water column is based on observations that the recycling of this element in particulate form while sinking down the water column is much lower than for the other nutrients, such that most of its remineralization is confined to the seafloor (Broecker and Peng, 1982; Dugdale et al., 1995).

The dynamics of DIC are given by photosynthesis and respiration of the organisms considered and calcification and dissolution of calcite:

$$
\begin{aligned}
\left.\frac{\partial O_{\mathbb{C}}}{\partial t}\right|_{\text {bgc }} & =\left.\frac{\partial B_{\mathbb{C}}}{\partial t}\right|_{\text {resp }}+\left.\sum_{\psi} \frac{\partial \stackrel{\psi}{P_{\mathbb{C}}}}{\partial t}\right|_{\text {resp }}+\left.\sum_{\Psi} \frac{\partial Z_{\mathbb{C}}}{\partial t}\right|_{\text {resp }} \\
& -\left.\sum_{\psi} \frac{\partial \stackrel{\chi}{P}_{\mathbb{C}}}{\partial t}\right|_{\text {gpp }}+\left.\frac{\partial{ }^{\text {calc }}}{\partial t}\right|_{\text {dis }}-\left.\frac{\partial L_{\mathbb{C}}^{\text {calc }}}{\partial t}\right|_{\text {calc }},
\end{aligned}
$$

where the respiration terms $\left.\frac{\partial B_{\mathbb{C}}}{\partial t}\right|_{\text {resp }},\left.\frac{\partial \Psi_{\mathbb{C}}^{\psi}}{\partial t}\right|_{\text {resp }}$ and $\left.\frac{\partial Z_{\mathbb{C}}}{\partial t}\right|_{\text {resp }}$ are given in Eqs. (50), (12), (35) and (43), synthesis of carbon is given in Eq. (8), the dissolution of calcite is given in Eq. (99) and precipitation of DIC into calcite is given by the sum of the calcification terms

$$
\left.\frac{\partial{ }^{\text {calc }} L_{\mathbb{C}}}{\partial t}\right|_{\text {calc }}=\left.\frac{\partial L_{\mathbb{C}}^{\text {calc }}}{\partial t}\right|_{\text {mort }}+\left.\frac{\partial L_{\mathbb{C}}^{\text {calc }}}{\partial t}\right|_{\text {graz }}+\left.\frac{\partial L_{\mathbb{C}}^{\text {calc }}}{\partial t}\right|_{\text {sed }}
$$

given in Eqs. (96), (97), and (98).

Rates of change of oxygen are implied from the corresponding carbon fluxes converted by stoichiometric factors taking into account different efficiencies for respiration $\stackrel{\text { resp }}{p}$ and photosynthesis $\stackrel{\text { syn }}{p_{\mathbb{O}}}$.

The pelagic oxygen cycle is reduced to the consumption of dissolved oxygen in respiration (Eqs. 50, 12, 35, and 43) and the production of dissolved oxygen in photosynthesis (Eq. 8):

$$
\begin{aligned}
\left.\frac{\partial O_{\mathbb{O}}}{\partial t}\right|_{\mathrm{bgc}} & =-\left.\stackrel{\operatorname{resp}}{p} \frac{\partial B_{\mathbb{C}}}{\partial t}\right|_{\text {resp }}-\left.\stackrel{\text { resp }}{p} \sum_{\Psi} \frac{\partial \stackrel{\psi}{P_{\mathbb{C}}}}{\partial t}\right|_{\text {resp }} \\
& -\left.P_{\mathbb{O}}^{\operatorname{resp}} \sum_{\Psi} \frac{\partial Z_{\mathbb{C}}}{\partial t}\right|_{\text {resp }}+\left.\stackrel{\text { syn }}{p} \underset{\mathbb{O}}{ } \sum_{\psi} \frac{\partial \stackrel{\chi}{P}_{\mathbb{C}}}{\partial t}\right|_{\mathrm{gpp}} .
\end{aligned}
$$

\subsection{The carbonate system}

The model for the carbonate system incorporated into ERSEM was introduced in Blackford and Burkill (2002) and further developed in Blackford and Gilbert (2007) and Artioli et al. (2012). In this model, the speciation of carbon 
is calculated from dissolved inorganic carbon $O_{\mathbb{C}}$, total alkalinity $\mathbb{A}_{\text {tot }}$ (which can be computed diagnostically, semidiagnostically, or prognostically; see below), and total boron $\mathbb{B}_{\text {tot }}$ (which is calculated from a linear regression of salinity). It assumes chemical equilibrium between the inorganic carbon species justified by the fast reaction timescales of the underlying chemical reaction compared to the biological and physical rates on the spatial scales the model operates on. The comprehensive set of equations to describe the carbonate system and ways to solve it given specific subsets of known quantities have been extensively described elsewhere (Dickson et al., 2007; Zeebe and Wolf-Gladrow, 2001); here, we use a simplified set omitting the components that contribute less under general seawater conditions (Takahashi et al., 1982).

The three quantities $O_{\mathbb{C}}, \mathbb{A}_{\text {tot }}$, and $\mathbb{B}_{\text {tot }}$ are used to derive the partial pressure of carbon dioxide $\mathrm{PCO}_{2}$, carbonic acid, carbonate, and bicarbonate concentrations $\left(c_{\left[\mathrm{H}_{2} \mathrm{CO}_{3}\right]}\right.$, $c_{\left[\mathrm{CO}_{3}^{2-}\right]}$, and $\left.c_{\left[\mathrm{HCO}_{3}^{-}\right]}\right)$and $\mathrm{pH}$ (using the seawater scale) at chemical equilibrium. These utilize the four equilibrium constants for solubility of carbon dioxide and for the dissociation of carbonic acid, bicarbonate, and boric acid derived from empirical environmental relationships (Millero, 1995; Mehrbach et al., 1973; Weiss, 1974; Dickson, 1990) that are detailed in the Supplement for reference. The resulting set of equations to solve is then given by

$$
\begin{aligned}
& O_{\mathbb{C}}=c_{\left[\mathrm{CO}_{3}^{2-}\right]}+c_{\left[\mathrm{HCO}_{3}^{-}\right]}+c_{\left[\mathrm{CO}_{2}^{*}\right]}, \\
& \mathbb{A}_{\text {tot }}=c_{\left[\mathrm{HCO}_{3}^{-}\right]}+2 c_{\left[\mathrm{CO}_{3}^{2-}\right]}+c_{\left[\mathrm{B}(\mathrm{OH})_{4}^{-}\right]} \text {, } \\
& \mathbb{B}_{\text {tot }}=c_{\left[\mathrm{B}(\mathrm{OH})_{3}\right]}+c_{\left[\mathrm{B}(\mathrm{OH})_{4}^{-}\right]} \text {, } \\
& c_{\mathrm{B}(\mathrm{OH})_{3}}=\frac{{ }^{c}\left[\mathrm{H}^{+}\right]^{c}\left[\mathrm{~B}(\mathrm{OH})_{4}^{-}\right]}{k_{\mathrm{b}}}, \\
& c_{\mathrm{CO}_{2}^{*}}=\frac{{ }^{c}\left[\mathrm{H}^{+}\right]^{c}\left[\mathrm{HCO}_{3}^{-}\right]}{k_{1}}, \\
& c_{\mathrm{HCO}_{3}^{-}}=\frac{{ }^{c}\left[\mathrm{H}^{+}\right]^{c}\left[\mathrm{CO}_{3}^{2-}\right]}{k_{2}}, \\
& \mathrm{pH}=-\log _{10}\left(c_{\left[\mathrm{H}^{+}\right]}\right), \\
& \mathrm{P}_{\mathrm{CO}_{2}}=k_{0} c\left[\mathrm{CO}_{2}^{*}\right] \text {. }
\end{aligned}
$$

The system is solved using the HALTAFALL algorithm (Ingri et al., 1967) by using the equilibrium relations Eqs. (126) to (128) to eliminate the unknowns $c_{\left[\mathrm{B}(\mathrm{OH})_{3}\right]}$, $c_{\left[\mathrm{CO}_{2}^{*}\right]}$ and $c_{\left[\mathrm{HCO}_{3}^{-}\right]}$. The balance equations for DIC and total boron are then used to express $c_{\left[\mathrm{CO}_{3}^{2-}\right]}$ and $c_{\left[\mathrm{B}(\mathrm{OH})_{4}^{-}\right]}$in the balance equation for alkalinity (Eq. 124) as functions of the only remaining unknown $c_{\left[\mathrm{H}^{+}\right]}$. This equation is solved for the logarithm of the unknown variable (allowing only positive real numbers as solution) applying a combination of the bisection method to narrow down the solution to a sufficiently small interval in $c_{\left[\mathrm{H}^{+}\right]}$to permit linear approximation followed by the secant method reducing the solution residual to the desired tolerance.

Calcite saturation is computed from the product of calcium and carbonate concentrations $\left(c_{\left[\mathrm{Ca}^{2+}\right]}\right.$ and $\left.c_{\left[\mathrm{CO}_{3}^{2-}\right]}\right)$ divided by their product in chemical equilibrium $k_{\text {calc }}$

$\Omega_{\text {calc }}=\frac{{ }^{c}\left[\mathrm{Ca}^{2+}\right]^{C}\left[\mathrm{CO}_{3}^{2-}\right]}{k_{\text {calc }}}$.

The variability of this ratio is dominated by ${ }^{c}\left[\mathrm{CO}_{3}^{2-}\right]$ as $c_{\left[\mathrm{Ca}^{2+}\right]}$ is nearly constant in seawater (Kleypas et al., 1999) and therefore fixed in the model at the oceanic mean value of $0.01028 \mathrm{~mol} \mathrm{~kg}^{-1}$.

Similarly, the aragonite saturation state is determined by the equation

$\Omega_{\text {calc }}=\frac{{ }^{c}\left[\mathrm{Ca}^{2+}\right]^{c}\left[\mathrm{CO}_{3}^{2-}\right]}{k_{\text {arag }}}$.

Two different modes to compute total alkalinity are provided with the model.

- A diagnostic mode that computes alkalinity from salinity or salinity and temperature. This mode is nonconservative and the field of alkalinity is recomputed at each time step without physical transport. It does not include changes to alkalinity by the biogeochemical processes of the model.

- A prognostic model that includes biogeochemical changes to alkalinity. It is fully conservative and adds a state variable for alkalinity that is subject to physical transport.

As a third semi-diagnostic option, these two modes can be combined as a sum by setting the prognostic alkalinity state to 0 , so that the diagnostic mode provides the background field and the prognostic mode gives a trace of the contribution of biogeochemical processes to the total alkalinity.

The recommended option is the semi-diagnostic option for coastal applications and shelf seas, where reliable and robust regressions exist, or the fully prognostic mode, where no single reliable regression is available, e.g. in global simulations. (For further details the reader is referred to Artioli et al., 2012.)

The changes in alkalinity due to biological processes are given by sources and sinks of phosphate, oxidized nitrogen and ammonium, as well as calcification and dissolution of calcite: 


$$
\begin{aligned}
\left.\frac{\partial A_{\text {bio }}}{\partial t}\right|_{\text {bgc }}= & \left.\frac{\partial N_{\mathbb{N}}^{\text {amm }}}{\partial t}\right|_{\text {bgc }}+\left.2 \frac{\partial L_{\mathbb{C}}}{\partial t}\right|_{\text {diss }} \\
& -\left.\frac{\partial N_{\mathbb{P}}}{\partial t}\right|_{\text {bgc }}-\left.\frac{\partial N_{\mathbb{N}}}{\partial t}\right|_{\text {bgc }}-\left.2 \frac{\partial L_{\mathbb{C}}}{\partial t}\right|_{\text {calc }}
\end{aligned}
$$

In three-dimensional simulations, these changes are accompanied by the effect of riverine inputs (see Artioli et al., 2012).

The different variants of alkalinity regressions available from the scientific literature (Borges and Frankignoulle, 1999; Bellerby et al., 2005; Millero et al., 1998; Lee et al., 2006), the total boron regression, and the empirical equilibrium constants $k$ are given in the Supplement.

\subsection{Light extinction}

Light in the water column is attenuated according to the Beer-Lambert formulation computing PAR as

$E_{\mathrm{PAR}}=q_{\mathrm{PAR}} I_{\mathrm{surf}} e^{\int_{0}^{z} K_{d}(\xi) \mathrm{d} \xi}$

where $I_{\text {surf }}$ is the shortwave radiation at sea-surface level, $q_{\text {PAR }}$ is a parameter for the photosynthetically active fraction, and $K_{d}$ is the spatially varying attenuation coefficient. The latter incorporates light attenuation by the modelled living and non-living optically active components as well as background extinction due to clear seawater and other components not explicitly modelled. Two alternative models are available for the computation of $K_{d}$.

1. A model based on mass-specific attenuation coefficients for the relevant functional types, non-modelled forms of inorganic matter, and the background attenuation of clear seawater; this model is used in previous ERSEM versions (Blackford et al., 2004) and is the default choice.

2. A model based on broadband inherent optical properties (absorption and backscatter), activated by the IOPMODEL preprocessing definition.

For the default model based on specific attenuation coefficients, $K_{d}$ is computed according to

$$
K_{d}=\sum_{\chi} \lambda_{P} \underset{P}{\stackrel{\chi}{P_{\mathbb{C}}}}+\sum_{\Psi} \lambda_{R} \stackrel{\Psi}{R_{\mathbb{C}}}+\lambda_{R_{\text {susp }}} R_{\text {susp }}+\Lambda_{\text {sea }},
$$

where $\lambda$ are the specific attenuation coefficients of the optically active components, i.e. the phytoplankton types $\chi$ and the particulate organic matter types $\Psi . \Lambda_{\text {sea }}$ is the background attenuation of seawater and $R_{\text {susp }}$ is the concentration of non-modelled optically active substances, mostly suspended matter.
The model based on inherent optical properties (activated by the preprocessing switch IOPMODEL) uses the light attenuation model proposed in Lee et al. (2005):

$K_{d}=\left(1+0.005 \theta_{\mathrm{zen}}\right) a+4.18\left(1.0-0.52 e^{-10.8 a}\right) b_{\mathrm{b}}$,

where $\theta_{\text {zen }}$ is the zenith angle at the given time and location. Absorption $a$ and backscatter $b_{\mathrm{b}}$ are composed as

$a=\sum_{\chi} a_{P}^{*} \stackrel{\chi}{P_{\mathcal{C}}}+\sum_{\Psi} a_{\Psi}^{*} \stackrel{\Psi}{R_{\mathbb{C}}}+a_{M_{\text {susp }}}+a_{\text {sea }}$,

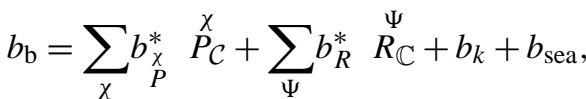

with $a^{*}$ and $b^{*}$ being the mass-specific absorption and backscatter coefficients of the respective components, $a_{\text {sea }}$ and $b_{\text {sea }}$ being the broadband absorption and backscatter of clear seawater, $a_{M_{\text {susp }}}$ the constant absorption of nonmodelled suspended matter, and $b_{k}$ a constant amount of background backscatter in the water column.

In both optical models the attenuation of optically active matter that is not modelled by ERSEM ( $R_{\text {susp }}$, mostly inorganic suspended particulate matter) can be provided homogeneously through a namelist parameter or spatially variable through the physical driver by filling and updating the ESS variable.

The combination of the attenuation of particulate organic matter and the non-modelled particles may be provided externally through the physical driver using the preprocessing definition ADYTRACER. This option introduces the state variable $a_{\text {ady }}$ and Eq. (136) reduces to

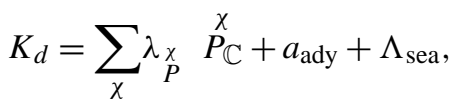

or in case of the model based on inherent optical properties

$a=\sum_{\chi} a_{\Psi}^{*} \stackrel{\chi}{P_{\mathcal{C}}}+a_{\text {ady }}+a_{\text {sea }}$,

$b_{\mathrm{b}}=\sum_{\chi} b_{\mathrm{b}, P}^{*} \stackrel{\chi}{P}_{\mathcal{C}}+b_{\mathrm{b}, k}+b_{\mathrm{b}, \mathrm{sea}}$,

neglecting the backscatter component of particulate and nonmodelled matter (see Eqs. 138 and 139).

The two models can be calibrated to give comparable results, but the latter formulation based on inherent properties has the advantage of being based on quantities that are frequently measured, which helps in constraining the parametrization and validation, and enables the direct assimilation of optical data.

\subsection{Gravitational sinking}

The sinking of model states is incorporated using a simple upwind scheme for the equation

$$
\left.\frac{\partial c_{\mathrm{p}}}{\partial t}\right|_{\mathrm{sed}}=\stackrel{c}{\mathrm{p}}_{\mathrm{sed}} \cdot \frac{\partial c_{\mathrm{p}}}{\partial z}
$$


and adding the resulting rate to the biogeochemical rates that are passed to the physical driver for integration.

The sedimenting states in the model are given by the par-

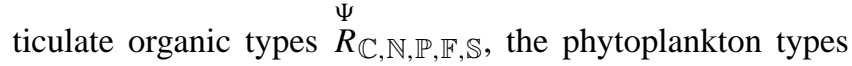
$\stackrel{\chi}{P}_{\mathbb{C}, \mathbb{N}, \mathbb{P}, \mathbb{F}, \mathbb{S}, \mathcal{C}}$ and calcite $\stackrel{\text { calc }}{L}$. Sinking velocities are constant velocities $\stackrel{\psi}{w}_{0}$ for each particulate matter type $\psi$, while for the phytoplankton states $\chi$ they are composed of a constant velocity complemented by a variable component subject to nutrient limitation beyond the threshold $\stackrel{\chi}{p}_{\text {sink }}$ :

$$
\stackrel{\chi}{w}_{\text {sed }}=\stackrel{\chi}{w}_{0}+\stackrel{\chi}{w}_{\text {lim }} \max \left(0, \stackrel{\chi}{p}_{\text {sink }}-\stackrel{\chi}{l}_{\langle\mathbb{N P P}\rangle}\right)
$$

\section{The benthic system}

The benthic model in ERSEM is predicated on muddy sediments of the continental shelf, including zoobenthos, bacteria, different forms of organic matter, and implicit vertical distribution of material within the seabed. It explicitly describes the main functions of the sediment such as benthic predation, decomposition and recycling of organic matter, bioirrigation, and bioturbation. As an alternative to using a full benthic model, the benthic-pelagic interface can be described by a simple benthic closure given in Sect. 5.1.5. This scheme adsorbs depositing particulate matter and phytoplankton and returns dissolved inorganic nutrients and carbon to the water column at a given timescale, reducing the sediments to a simple buffer layer of organic matter recycling that however does not involve any explicit benthic processes. It is computationally considerably lighter compared to the full model, but the computational effort in both cases is negligible compared to the pelagic component. While the full benthic model is more adequate for shelf-sea applications that are dominated by the sediment type it represents with a close connection to the productive upper ocean, the simplified closure scheme is more suitable in deep domains under oligotrophic conditions, where the sediment processes are of lesser importance.

\subsection{Benthic model structure}

The full benthic model is a simplified version (Blackford, 1997; Kohlmeier, 2004) of the more complex original model introduced in the original version of ERSEM (Ruardij and Van Raaphorst, 1995; Ebenhöh et al., 1995) assuming nearequilibrium conditions for the inorganic components. Organisms are distinguished in classes on a more functional and less size oriented base than in the pelagic part.

The model includes the functional types of aerobic and anaerobic bacteria as decomposers of organic material, three types of benthic predators (suspension feeders, deposit feeders, and meiobenthos), dissolved organic matter, and three forms of particulate detritus classified according to their availability and decomposition timescales into degradable, available refractory, and buried refractory matter.

Benthic state variables are vertically integrated contents (in mass per area) whose vertical distributions are constrained by the following simplifying assumptions: three distinct layers are considered in the model, a top, aerobic layer that is oxygenated and delimited by the horizon of dissolved oxygen, an intermediate oxidized layer with no free oxygen but oxidized nitrogen available (also referred to as the denitrification layer) and delimited by the horizon of oxidized nitrogen, and a completely anoxic deep sediment layer. Given its very shallow penetration into the sediments, for simplicity, dissolved organic matter is also assumed to be restricted to the aerobic layer. Below these layers, limited by the total depth horizon of the model, no biogeochemical processes take place, and only buried refractory matter exists.

The chemical components of the types are identical to the pelagic part consisting of carbon, nitrogen, phosphorus, silicate, and iron; the silicate and iron cycles are simplified, bypassing the living functional types, in a similar manner to the pelagic part of the model. The silicate contained in detritus is remineralized implicitly into inorganic form in the sediments, while the iron in detritus is directly recycled and returned to the water column.

The vertical distribution of dissolved inorganic and particulate organic matter is crucial in determining the availability of food and resources to the benthic organisms. It is implicitly resolved assuming near-equilibrium conditions for the inorganic components determining the diffusion rate with the overlying water body for the inorganic forms and assumes exponentially decaying distributions for particulate organic matter. The vertical dynamics of these distributions are described by dedicated state variables that describe the structure of the sediments. These are given by the oxygen horizon (the lower limit of the oxygenated layer and the upper limit of the denitrification layer), the oxidized nitrogen horizon (the lower limit of the denitrification layer and the upper limit of the strictly anoxic layer), and the mean penetration depths for available refractory carbon, nitrogen, and phosphorus and degradable carbon, nitrogen, phosphorus, and silicate.

A complete list of benthic state variables is given in Table 3 .

\subsection{Implicit vertical distribution of inorganic states in the benthos}

In order to determine the dynamics of the oxygen and oxidized nitrogen horizons as well as the inorganic fluxes across the seabed (Sect. 5.1.3), the inorganic components of the benthos are assumed to be close to their equilibrium distributions, in which all source and sink terms of the porewater concentrations of the inorganic components $c_{\mathrm{pw}}$ inside the sediments are perfectly balanced by diffusion: 
Table 3. Benthic functional types and their components (squared brackets indicate option states) - chemical components: $\mathbb{C}$ carbon, $\mathbb{N}$ nitrogen, $\mathbb{P}$ phosphorus, $\mathbb{F}$ iron, $\mathbb{S}$ silicate.

\begin{tabular}{|c|c|c|}
\hline Symbol & Code & Description \\
\hline DEPO & & \\
\hline$Y_{\mathbb{C}}$ & $\mathrm{Y} 2 \mathrm{C}$ & Deposit feeders \\
\hline $\begin{array}{c}\text { SUSP } \\
Y_{\mathbb{C}}\end{array}$ & Y3C & Suspension feeders \\
\hline MEIO & & \\
\hline$Y_{\mathbb{C}}$ & Y4C & Meiobenthos \\
\hline$H_{\mathbb{C}}$ & $\mathrm{H} 1 \mathrm{C}$ & Aerobic bacteria \\
\hline $\begin{array}{c}\text { anaer } \\
H_{\mathbb{C}}\end{array}$ & $\mathrm{H} 2 \mathrm{C}$ & Anaerobic bacteria \\
\hline $\begin{array}{l}\text { dis } \\
Q_{\mathbb{C}} \\
\text { degr }\end{array}$ & Q1C & Dissolved organic matter \\
\hline$Q_{\mathbb{C}, \mathbb{N}, \mathbb{P}[, \mathbb{F}]}$ & $\mathrm{Q} 6 \mathrm{c}, \mathrm{n}, \mathrm{p}[, \mathrm{f}], \mathrm{s}$ & Degradable organic matter \\
\hline$\underset{\text { bur }}{Q} \mathbb{C}, \mathbb{N}, \mathbb{P}, \mathbb{S}$ & $\mathrm{Q} 7 \mathrm{c}, \mathrm{n}, \mathrm{p}, \mathrm{s}$ & Refractory organic matter \\
\hline$Q_{\mathbb{C}, \mathbb{N}, \mathbb{P}}$ & $\mathrm{Q} 17 \mathrm{c}, \mathrm{n}, \mathrm{p}$ & Buried organic matter \\
\hline$C_{\mathbb{C}}$ & {$[\mathrm{bL} 2 \mathrm{c}]$} & Calcite \\
\hline$G_{\mathbb{O}}$ & G2O & Dissolved oxygen \\
\hline$G_{\mathbb{C}}$ & G3C & Dissolved inorganic carbon (DIC) \\
\hline$G_{\mathbb{N}}$ & $\mathrm{G} 4 \mathrm{n}$ & Dinitrogen \\
\hline$\underset{\mathrm{ox}}{K_{\mathbb{P}}}$ & $\mathrm{K} 1 \mathrm{p}$ & Phosphate \\
\hline $\begin{array}{l}K_{\mathbb{N}} \\
\mathrm{amm}\end{array}$ & K3n & Oxidized nitrogen \\
\hline$K_{\mathbb{N}}$ & $\mathrm{K} 4 \mathrm{n}$ & Ammonium \\
\hline $\begin{array}{c}K_{\mathbb{S}} \\
\text { oxy }\end{array}$ & $\mathrm{K} 5 \mathrm{~s}$ & Silicate \\
\hline$D$ & $\mathrm{D} 1 \mathrm{~m}$ & Depth of oxygen horizon \\
\hline $\begin{array}{c}\text { denit } \\
D\end{array}$ & $\mathrm{D} 2 \mathrm{~m}$ & Depth of oxidized nitrogen horizon \\
\hline $\begin{array}{r}\operatorname{refr}_{\mathbb{C}} \\
D\end{array}$ & D3m & Average penetration depth of refractory carbon \\
\hline $\begin{array}{r}\operatorname{refr}_{\mathbb{N}} \\
D\end{array}$ & $\mathrm{D} 4 \mathrm{~m}$ & Average penetration depth of refractory nitrogen \\
\hline $\begin{array}{c}\operatorname{refr}_{\mathbb{P}} \\
D \\
\operatorname{degr}_{\mathbb{C}}\end{array}$ & $\mathrm{D} 5 \mathrm{~m}$ & Average penetration depth of refractory phosphorus \\
\hline$\underset{\operatorname{degr}_{\mathbb{N}}}{D}$ & D $6 \mathrm{~m}$ & Average penetration depth of degradable carbon \\
\hline$\stackrel{D}{D}$ & $\mathrm{D} 7 \mathrm{~m}$ & Average penetration depth of degradable nitrogen \\
\hline $\begin{array}{c}D \\
\operatorname{degr}_{\mathbb{S}}\end{array}$ & D 8m & Average penetration depth of degradable phosphorus \\
\hline$\stackrel{\operatorname{degr}_{\mathbb{S}}}{D}$ & D9m & Average penetration depth of degradable silicate \\
\hline
\end{tabular}

$v_{\text {idiff }} \frac{\partial^{2} c_{\mathrm{pw}}}{\partial \zeta^{2}}=\left.\frac{1}{\Delta d} \frac{\partial c_{\mathrm{b}}}{\partial t}\right|_{\mathrm{bgc}}$,

where $c_{\mathrm{b}}$ is the layer content. This partial differential equation has a general parabolic solution in $\zeta$ taking the sourcesink term $\left.\frac{\partial c_{\mathrm{b}}}{\partial t}\right|_{\mathrm{bgc}}$ as a fixed equilibrium rate independent of time. This is a reasonable assumption when the diffusive rates are significantly faster than the biogeochemical processes ( $v_{\text {idiff }}$ is the diffusivity of dissolved inorganic components in the benthos depending on bioirrigation; see Eq. 215). The equations apply to each of the three sediment layers and the resulting system of piece-wise parabolic continuous pro- files can be solved using two boundary conditions per layer: the surface concentration at the upper boundary starting with the sediment surface concentration and the flux across the lower boundary which is equal to the sum of all source and sink processes below the layer under consideration (by definition, no fluxes of dissolved matter can occur across the bottom of the sediments, so that all sources and sinks have to be compensated for from above).

The sediment surface concentration $c_{\text {bed }}$ required as a boundary condition for the production-diffusion balance above is generally not equal to the concentration at the centre of the lowest pelagic discretization cell $c_{\mathrm{p}}$, as diffusion across 
the sediment surface will be attenuated by the bottom boundary layer. In the simplest case the difference between cell centre and sediment surface concentrations can be estimated assuming a linear diffusive flux as positively proportional to the biogeochemical net change in the sediments. However, a problem arises for this formulation when the sediments act as a net sink, as the calculated differences may exceed the cell centre concentration, suggesting negative concentrations at the sediment interface. Therefore, for negative net sinks in the sediments the formulation suggested by Patankar (1980) and Burchard et al. (2003) is applied, leading to the equation

$c_{\text {bed }}=\left\{\begin{array}{lll}c_{\mathrm{p}}+\left.p_{\text {vmix }} \frac{\partial c_{\mathrm{b}}}{\partial t}\right|_{\mathrm{bgc}} & \text { if } & \left.\frac{\partial c_{\mathrm{b}}}{\partial t}\right|_{\mathrm{bgc}}>0, \\ c_{\mathrm{p}} \frac{c_{\mathrm{p}}}{c_{\mathrm{p}}-\left.p_{\text {vmix }} \frac{\partial c_{\mathrm{b}}}{\partial t}\right|_{\mathrm{bgc}}} & \text { if } & \left.\frac{\partial c_{\mathrm{b}}}{\partial t}\right|_{\mathrm{bgc}}<0,\end{array}\right.$

where $p_{\text {vmix }}$ is an inverse mixing velocity constant.

The resulting equilibrium porewater concentrations $\widetilde{c}_{\mathrm{pw}}$ in each layer are converted into the full equilibrium layer contents using the layer thickness and the conversion factor

$\nu_{\mathbb{N}, \mathbb{P}}=p_{\text {poro }} p_{\text {ads }}$,

where $p_{\text {poro }}$ and $p_{\text {ads }}$ are porosity and adsorption factors that may vary spatially in the case of porosity and adsorption of phosphorus, while they are constants for all other adsorptions.

The dynamics of the oxygen and oxidized nitrogen horizons are determined by a relaxation towards their equilibrium values $\stackrel{\text { oxy }}{d_{\text {eq }}}$ and $\stackrel{\text { denit }}{d_{\text {eq }}}$, which are the depths where the porewater equilibrium concentrations are 0 . Their time evolution is then described by

$$
\begin{aligned}
& \frac{\partial D}{\partial t}=\frac{1}{\tau_{\text {oxy }}}\left(\begin{array}{c}
\text { oxy } \\
d_{\text {eq }}-\stackrel{\text { oxy }}{D}
\end{array}\right), \\
& \frac{\partial D}{\partial t}=\frac{1}{\tau_{\text {denit }}}\left(\begin{array}{l}
\text { denit } \\
d_{\text {eq }}-\stackrel{\text { denit }}{D}
\end{array}\right),
\end{aligned}
$$

where $\tau_{\mathrm{ox}}$ and $\tau_{\text {denit }}$ are the respective relaxation timescales.

\subsection{Implicit vertical distribution of organic matter in the benthos}

The penetration of organic matter type $\psi$ into the sediments is assumed as exponential decay of a concentration $\stackrel{\psi}{c}(\zeta)$ from a sediment surface value $c_{0}$ as a function of the $e$ folding depth $\lambda$ :

${ }^{\psi}(\zeta)=c_{0} e^{-\frac{\zeta}{\lambda}}$

Total content ${ }^{\psi} c_{\mathrm{b}}$ is then given by the integral

$\psi_{\mathrm{b}}={ }_{c_{0}} \int_{0}^{d_{\mathrm{tot}}} e^{-\frac{\zeta}{\lambda}} \mathrm{d} \zeta$ and the penetration depth $\stackrel{\psi}{D}$ of matter $\psi$ is defined accordingly as

$\stackrel{\psi}{D}=\frac{1}{\psi}{ }_{c_{\mathrm{b}}}^{\psi} c_{0} \int_{0}^{d_{\mathrm{tot}}} \zeta e^{-\frac{\zeta}{\lambda}} \mathrm{d} \zeta$.

For $d_{\mathrm{tot}} \rightarrow \infty$ the two integrals of Eqs. (151) and (152) yield

$\lambda=\stackrel{\psi}{D}=\frac{\psi}{\psi} \frac{c_{\mathrm{b}}}{c_{0}}$,

i.e. the mean penetration depth is given by the $e$-folding depth of the distribution function:

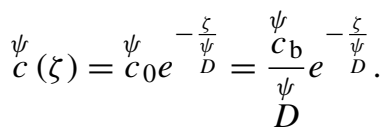

The change of penetration depth due to vertically distributed sources and sinks $f(\zeta)$ can then be calculated by the formula:

$\frac{\mathrm{d} D}{\mathrm{~d} t}=\int_{0}^{\infty}(\zeta-D) \frac{f(\zeta)}{c_{\mathrm{b}}} \mathrm{d} \zeta$.

As the model is not vertically explicit, but, based on the model assumptions, processes can be attributed to layers (e.g. activity of aerobic bacteria to the aerobic layer), the changes $\mathcal{F}_{i}$ caused in a given layer can be attributed to discrete depth levels being the centre of the layer $\zeta_{i}$.

The changes of penetration depth due to source and sink terms are complemented by the physical displacement of organic matter by the process of bioturbation, so that the total change is given by the equation:

$\frac{\partial \stackrel{\psi}{D}}{\partial t}=\sum_{i}\left(d_{i}-\stackrel{\psi}{D}\right) \underset{c_{\mathrm{b}}}{\psi} \frac{f_{i}}{\partial t}+\frac{\partial \stackrel{\psi}{D}}{\left.\right|_{\mathrm{bturb}}}$.

Bioturbation smoothes the concentration gradient and is therefore implemented as diffusive flux proportional to the difference in concentrations between 0 and a bioturbation length scale $\delta_{\text {bturb}}$ :

$\left.\frac{\partial \stackrel{\psi}{D}}{\partial t}\right|_{\text {bturb }}=\frac{\nu_{\text {bturb }}}{c_{\mathrm{b}}}\left(\stackrel{\psi}{c_{0}}-\stackrel{\psi}{c}\left(\delta_{\text {bturb }}\right)\right)$,

where $\nu_{\mathrm{bturb}}$ is the bioturbation diffusivity of particulate matter (Eq. 217). Still assuming that $\stackrel{\psi}{D} \ll d_{\text {tot }}$, this takes the form

$\left.\frac{\partial \stackrel{\psi}{D}}{\partial t}\right|_{\text {bturb }}=\frac{\nu_{\text {bturb }}}{\psi}\left(1-e^{-\frac{\delta_{\text {bturb }}}{\sim}}\right)$. 
The fraction of organic matter contained between two given depth levels can then be computed as

$\frac{\left.\psi_{c_{\mathrm{b}}}\right|_{d_{\mathrm{up}}} ^{d_{\mathrm{low}}}}{{ }_{c_{\mathrm{b}}}}=\frac{1}{\psi} \int_{c_{\mathrm{b}}}^{d_{\mathrm{low}}} \gamma(\zeta) \mathrm{d} \zeta=\frac{e^{-\frac{d_{\mathrm{up}}}{\psi}}-e^{-\frac{d_{\mathrm{low}}}{\psi}}}{1-e^{-\frac{d_{\mathrm{tot}}}{\nu}}}$,

where the total content was approximated as

${ }^{\psi} c_{\mathrm{b}}=\int_{0}^{d_{\mathrm{tot}}} \gamma(\zeta) d \zeta=\psi_{0} \stackrel{\psi}{D}\left(1-e^{-\frac{d_{\mathrm{tot}}}{\nu}}\right)$.

For consistency with the model assumptions and to avoid numerical issues the penetrations depths are constrained to values between $\stackrel{\psi}{D_{0}}$ and $d_{\text {tot }}$.

Dissolved organic matter is assumed to reside entirely in the oxygenated layer.

\subsection{Heterotrophic bacteria}

Benthic decomposers consist of aerobic bacteria living in the upper sediment layer down to the oxygen horizon and anaerobic bacteria living in the denitrification layer and anoxic layer. Their dynamics are summarized by the equations

$$
\begin{aligned}
& \left.\frac{\partial \stackrel{\chi}{H_{\mathbb{C}}}}{\partial t}\right|_{\text {bgc }}=\left.\frac{\partial \stackrel{\chi}{H_{\mathbb{C}}}}{\partial t}\right|_{\text {upt }}-\left.\frac{\partial \stackrel{\chi}{H_{\mathbb{C}}}}{\partial t}\right|_{\text {excr }}-\left.\frac{\partial \stackrel{x}{H_{\mathbb{C}}}}{\partial t}\right|_{\text {resp }} \\
& -\left.\frac{\partial \stackrel{\chi}{H}_{\mathbb{C}}}{\partial t}\right|_{\text {pred }}-\left.\frac{\partial \stackrel{\chi}{H}_{\mathbb{C}}}{\partial t}\right|_{\text {mort }}-\left.\frac{\partial \stackrel{\chi}{H}_{\mathbb{C}}}{\partial t}\right|_{\text {rel }},
\end{aligned}
$$

$$
\begin{array}{r}
\left.\frac{\partial \stackrel{\chi}{H}_{\mathbb{N}, \mathbb{P}}}{\partial t}\right|_{\text {bgc }}=\left.\frac{\partial \stackrel{\chi}{H}_{\mathbb{N}, \mathbb{P}}}{\partial t}\right|_{\text {upt }}-\left.\frac{\partial \stackrel{\chi}{H}_{\mathbb{N}, \mathbb{P}}}{\partial t}\right|_{\text {excr }} \\
\quad-\left.\frac{\partial \stackrel{\chi}{H}_{\mathbb{N}, \mathbb{P}}}{\partial t}\right|_{\text {pred }}-\left.\frac{\partial \stackrel{\chi}{H}_{\mathbb{N}, \mathbb{P}}}{\partial t}\right|_{\text {mort }}-\left.\frac{\partial \stackrel{\chi}{H}_{\mathbb{N}, \mathbb{P}}}{\partial t}\right|_{\text {rel }}
\end{array}
$$

Substrate mass-specific bacterial uptake is regulated by the sediment surface temperature, oxygen availability (in free or bound form) and the nutritional state of the substrate (through the regulating factors ${ }_{l}^{\chi},{ }_{l_{\mathbb{O}}}^{\chi}$, and $\stackrel{\chi}{l}_{\langle\mathbb{N P}\rangle}$, Eqs. 239, $252,247)$, and the amount of bacteria in the given location:

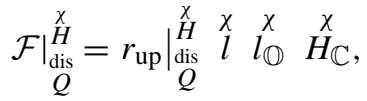

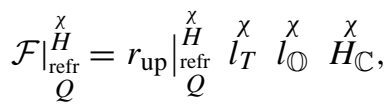

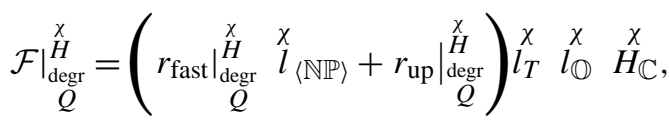

where $\left.r_{\text {up }}\right|_{Q} ^{\stackrel{x}{H}}$ are the bacteria and substrate mass-specific reference uptake rates. These are generally high for the dissolved form and low for refractory matter. Decomposition of degradable matter has a slow basal component comple-

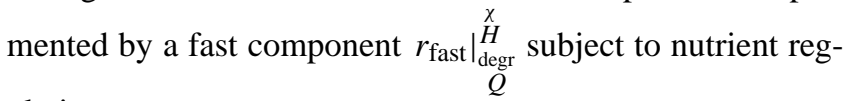
ulation.

To obtain the uptake rates, these substrate mass-specific rates are multiplied by the substrate concentrations available in the respective layer (given by Eq. 159):

$$
\left.\frac{\partial \stackrel{\chi}{H}_{\mathbb{C}}}{\partial t}\right|_{\text {upt }}=\left.\sum_{\psi} \mathcal{F}\right|_{\underset{\psi}{H}} ^{\stackrel{\chi}{\psi}} \underset{Q_{\mathbb{C}}^{\prime}}{d_{d_{\text {up }}}^{d_{\text {low }}}},
$$

where the layer limits $d_{\text {low }}, d_{\text {up }}$ are $0, \stackrel{\text { oxy }}{D}$ for aerobic bacteria and $\stackrel{\text { oxy }}{D}, d_{\text {tot }}$ for anaerobic bacteria. Aerobic bacteria feed on dissolved and particulate substrate, while anaerobic bacteria feed exclusively on the particulate form.

The uptake of organic nitrogen and phosphorus is enhanced by a nutrient preference factor $\stackrel{\chi}{p}_{\text {nup }}$ supported by observations that the relative nutrient content of benthic DOM decreases under bacteria production (van Duyl et al., 1993). It is complemented by the uptake of inorganic forms when organic matter is nutrient poor with respect to the fixed bacterial stoichiometric ratio. Inorganic uptake of nutrients by each bacteria type is regulated by Michaelis-Menten terms of the porewater inorganic nutrient content within the oxygenated or oxidized layer with the Redfield equivalent of carbon uptake as the half-saturation term:

$$
\begin{aligned}
& \left.\frac{\partial \stackrel{\chi}{H}_{\mathbb{N}, \mathbb{P}}}{\partial t}\right|_{\text {upt }}=\left.\left.\sum_{\psi} \stackrel{\chi}{p}_{\text {nup }} \mathcal{F}\right|_{Q} ^{\stackrel{\chi}{H}} Q_{\mathbb{N}, \mathbb{P}}^{\prime}\right|_{d_{\text {up }}} ^{d_{\text {low }}} \\
& +\left.\stackrel{\chi}{q}_{\text {ref }_{\mathbb{N}, \mathbb{P}: \mathbb{C}}} \frac{\partial \stackrel{x}{H}_{\mathbb{C}}}{\partial t}\right|_{\text {upt }}
\end{aligned}
$$

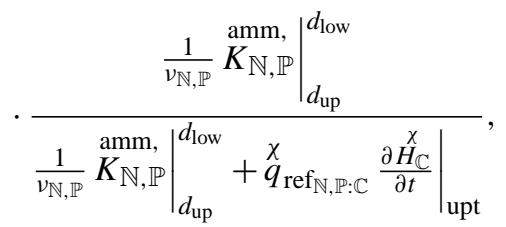


where $\left.\stackrel{\text { amm, }}{K_{\mathbb{N}}}\right|_{d_{\text {up }}} ^{d_{\text {low }}}$ and $\left.K_{\mathbb{P}}\right|_{d_{\text {up }}} ^{d_{\text {low }}}$ are the respective layer contents of ammonium or phosphate between the depths $d_{\text {up }}$ and $d_{\text {low }}$, and $\nu_{\mathbb{N}, \mathbb{P}}$ is a volume correction factor (Eq. 147) reducing the total layer content to the porewater content.

Anaerobic bacteria feeds on and excretes only in particulate form, so that the above rates are for gross uptake in the case of aerobic bacteria, followed by excretion in dissolved form, while for anaerobic bacteria they are net rates with no subsequent excretion. Excretion occurs at fixed fractions $\stackrel{\text { aer }}{q}$ dexcr,$\stackrel{a e r}{q}_{\text {rexcr }}$ of the aerobic bacteria uptake according to

$$
\begin{aligned}
& \left.\frac{\partial \stackrel{H}{H}_{\mathbb{C}, \mathbb{N}, \mathbb{P}}}{\partial t}\right|_{\text {excr }}=\left.\stackrel{\text { aer }}{q \text { dexcr }}_{\stackrel{\mathcal{F}}{\stackrel{\text { aer }}{H}} \stackrel{\text { degr }}{Q} Q_{\mathbb{C}, \mathbb{N}, \mathbb{P}}^{\prime}}\right|_{d_{\text {up }}} ^{d_{\text {low }}} \\
& +\left.\left.\stackrel{q}{q}_{\text {rexcr }} \mathcal{F}\right|_{\text {refr }} ^{\stackrel{\text { aer }}{H}} Q_{\mathbb{C}, \mathbb{N}, \mathbb{P}}^{\prime}\right|_{d_{\text {up }}} ^{\text {refr }},
\end{aligned}
$$

$\left.\frac{\partial \stackrel{\text { anaer }}{H} \mathbb{C}, \mathbb{N}, \mathbb{P}}{\partial t}\right|_{\text {excr }}=0$

Respiration of bacteria is given by activity respiration as a fraction of gross uptake $\stackrel{\chi}{q}_{\text {aresp }}$ and temperature regulated basal respiration at rest proportional to the bacteria biomass by the factor $\stackrel{\chi}{r}_{\text {resp }}$ :

$$
\left.\frac{\partial \stackrel{\chi}{H}_{\mathbb{C}}}{\partial t}\right|_{\text {resp }}=\left.\stackrel{\chi}{q}_{\text {aresp }} \frac{\partial \stackrel{\chi}{H}_{\mathbb{C}}}{\partial t}\right|_{\text {upt }}+\stackrel{\chi}{r}_{\text {resp }} \stackrel{\chi}{l_{T}} \quad \stackrel{\chi}{H_{\mathbb{C}}^{\prime}} .
$$

Bacterial mortality is fully regulated by oxygen (see Eq. 252) and proportional to the bacteria biomass by factor

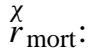

$$
\left.\frac{\partial \stackrel{\chi}{H}_{\mathbb{C}, \mathbb{N}, \mathbb{P}}}{\partial t}\right|_{\text {mort }}=\stackrel{\chi}{r}_{\text {mort }}\left(1-\chi_{\mathbb{l}}^{\chi}\right) \stackrel{\chi}{H^{\prime}} \mathbb{C , N , \mathbb { P }}
$$

where aerobic bacteria use oxygen in dissolved form while anaerobic bacteria satisfy their oxygen requirements from oxidized nitrogen.

Benthic bacteria are held at a fixed stoichiometric quota ${\stackrel{\chi}{\text { ref }_{\mathbb{N}, \mathbb{P}: \mathbb{C}}}}$, so that any chemical component flux in excess of the reference quota is released according to Eqs. (268) and (269), in dissolved form for the nutrients and in the form of organic matter for carbon.

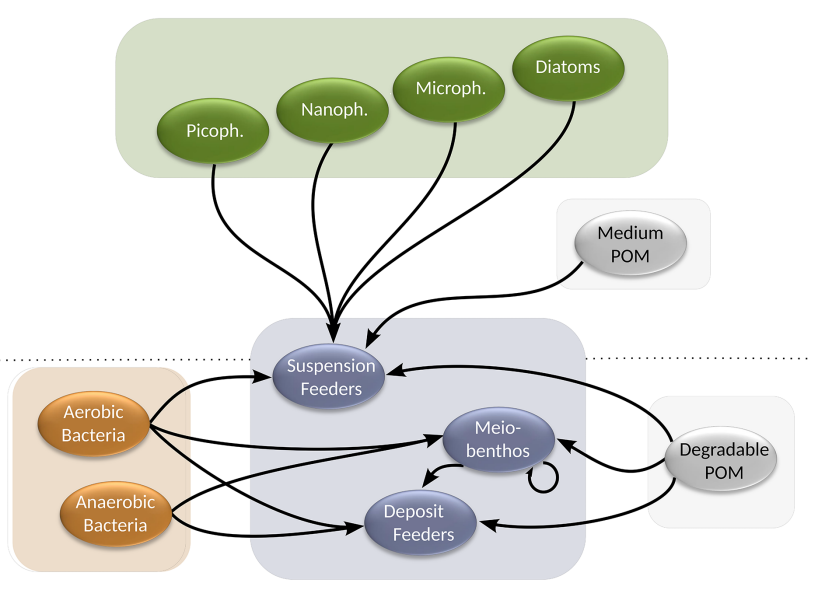

Figure 5. Benthic predators and their prey.

\subsection{Predators}

The general biogeochemical dynamics of the zoobenthos types $\chi$ are given by the equations

$$
\begin{array}{r}
\left.\frac{\partial Y_{\mathbb{C}}^{\chi}}{\partial t}\right|_{\text {bgc }}=\left.\frac{\partial Y_{\mathbb{C}}}{\partial t}\right|_{\text {upt }}-\left.\frac{\partial Y_{\mathbb{C}}}{\partial t}\right|_{\text {excr }}-\left.\frac{\partial Y_{\mathbb{C}}}{\partial t}\right|_{\text {resp }} \\
-\left.\frac{\partial Y_{\mathbb{C}}}{\partial t}\right|_{\text {pred }}-\left.\frac{\partial Y_{\mathbb{C}}}{\partial t}\right|_{\text {mort }}-\left.\frac{\partial Y_{\mathbb{C}}}{\partial t}\right|_{\text {rel }}, \\
\left.\frac{\partial \underline{Y}_{\mathbb{N}, \mathbb{P}}}{\partial t}\right|_{\text {bgc }}=\left.\frac{\partial \stackrel{\chi}{Y}, \mathbb{P}_{\mathbb{N}}}{\partial t}\right|_{\text {upt }}-\left.\frac{\partial Y_{\mathbb{N}, \mathbb{P}}}{\partial t}\right|_{\text {excr }}-\left.\frac{\partial \underline{Y}_{\mathbb{N}, \mathbb{P}}}{\partial t}\right|_{\text {rel }} \\
-\left.\frac{\partial \stackrel{\chi}{Y}, \mathbb{P}_{\mathbb{N}}}{\partial t}\right|_{\text {pred }}-\left.\frac{\partial Y_{\mathbb{N}, \mathbb{P}}}{\partial t}\right|_{\text {mort }}-\left.\frac{\partial Y_{\mathbb{N}, \mathbb{P}}}{\partial t}\right|_{\text {rel }} .
\end{array}
$$

The benthic predators considered in ERSEM are deposit feeders, suspension feeders and meiobenthos, distinguished by their prey fields and preferences, the depth section they live in and their respective metabolic rates. The prey fields available to each type are given in Fig. 5, where organic matter is scavenged only in the depth sections accessible to each predators given by three parameters as follows:

- suspension feeders: $0 \leqslant \zeta \leqslant \stackrel{\text { SUSP }}{d_{Y}}$,

- deposit feeders: ${ }^{\text {SUSP }} d_{Y} \leqslant \zeta \leqslant{ }^{\text {DEPO }}$

- meiobenthos: $0 \leqslant \zeta \leqslant d_{Y}$.

An additional parameter $d_{\text {SUSP }}$ indicates the range of suspension feeders into the water column assuming homogenous prey distribution over this scale. 
The total prey available to each zoobenthos type $\chi$ is composed of the individual prey types $\psi$ as

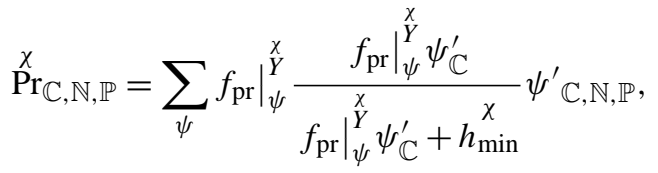

where $\left.f_{\mathrm{pr}}\right|_{\psi} ^{\chi}$ are the food preferences and $h_{\min }^{\chi}$ is a food halfsaturation constant limiting the detection capacity of predator $\chi$ of individual prey types similar to the zooplankton predation (Eq. 29). In contrast to the pelagic form, the detection capability for the benthic fauna is assumed to vary by food source assuming that benthic predators search their food more actively. The prey contents in the half-saturation term are consequently multiplied by the food preferences.

The prey mass-specific uptake capacity for each zooplankton type $\chi$ is then given by

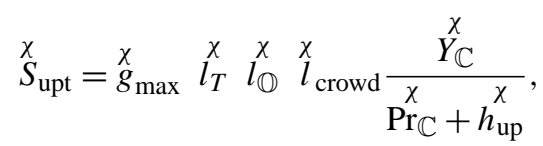

where $\stackrel{\chi}{g}_{\text {max }}$ is the maximum uptake capacity of each type at reference temperature, $l_{T}^{\chi}$ is the metabolic temperature response (Eq. 239), $\ell_{\mathbb{O}}^{\chi}$ is the limitation of oxygen (Eq. 250), $\stackrel{\chi}{l}_{\text {crowd }}$ is a growth limiting penalty function accounting for overcrowding effects (Eq. (267), absent for meiobenthos as this type is capable of feeding on itself), $h_{\mathrm{up}}^{\chi}$ is a predation efficiency limiting the chances of encountering the prey available $\left(\operatorname{Pr}_{\mathbb{C}}\right)$

Introducing the prey mass-specific fluxes from prey $\psi$ to predator $\stackrel{\chi}{Y}$

$$
\left.\mathcal{F}\right|_{\psi} ^{\stackrel{\chi}{Y}}=\stackrel{\mathcal{S}}{\text { upt }}_{\text {pr }} f_{\psi}^{\stackrel{\chi}{Y}} \frac{\left.f_{\mathrm{pr}}\right|_{\psi} ^{\underline{\chi}} \psi_{\mathbb{C}}^{\prime}}{\left.f_{\mathrm{pr}}\right|_{\psi} ^{\underline{Y}} \psi_{\mathbb{C}}^{\prime}+h_{\text {min }}^{\chi}}
$$

with $\left.f_{\mathrm{pr}}\right|_{\psi} ^{\stackrel{\chi}{Y}}$ being the food preference of predator $\stackrel{\chi}{Y}$ for prey $\psi$, and $h_{\min }^{\chi}$ being a half-saturation constant reflecting the detection capacity of predator $\chi$; the zooplankton uptake can then be written as

$$
\left.\frac{\partial \stackrel{\chi}{Y}_{\mathbb{C}, \mathbb{N}, \mathbb{P}}}{\partial t}\right|_{\text {upt }}=\left.\sum_{\psi} \mathcal{F}\right|_{\psi} ^{\stackrel{\chi}{Y}} \psi_{\mathbb{C}, \mathbb{N}, \mathbb{P}}^{\prime}
$$

Zoobenthos excretion is given by

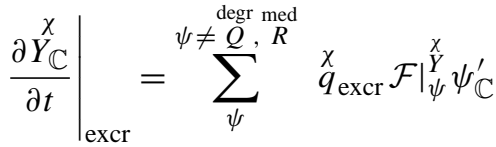

$$
\begin{aligned}
& +\left.\sum_{\psi}^{\psi=\stackrel{\text { degr }}{Q}, R_{R}^{\text {med }}}{\stackrel{\chi}{q_{\text {pexcr }}}} \mathcal{F}\right|_{\psi} ^{\chi} \psi_{\mathbb{C}}^{\prime}
\end{aligned}
$$

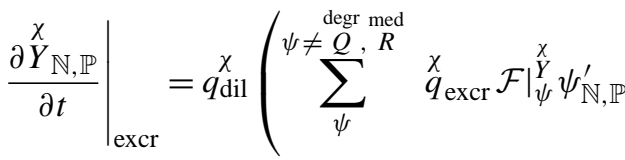

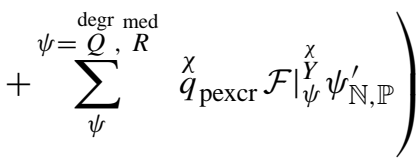

where $\stackrel{\chi}{q}_{\text {excr }}$ is a fixed proportion of gross uptake excreted and $\underset{\text { dil }}{\chi}$ an additional dilution coefficient taking into account a reduced amount of nutrients in the fecal pellets with respect to the uptake quota.

Respiration of zoobenthos is given by activity respiration as a fraction of net uptake $\stackrel{\chi}{q}_{\text {aresp }}$ and temperature regulated respiration at rest proportional to the zoobenthos biomass by the factor $\stackrel{\chi}{r}_{\text {resp }}$ :

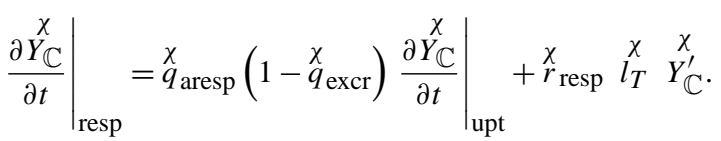

Zoobenthos mortality is regulated by temperature and oxygen and composed of a basal part enhanced under oxygen deficiency and cold temperatures by the factors $\stackrel{x}{r}$ mort $\mathbb{O}, \stackrel{x}{r}_{\text {mort } T}$ :

$$
\begin{aligned}
& \left.\frac{\partial \stackrel{\chi}{Y}_{\mathbb{C}, \mathbb{N}, \mathbb{P}}}{\partial t}\right|_{\text {mort }}=\left(\stackrel{\chi}{r}_{\text {mort }} \stackrel{\chi}{l_{T}}+\stackrel{\chi}{r}_{\text {mort } \mathbb{O}} \stackrel{\chi}{l_{T}}\left(1-\stackrel{\chi}{l}_{\mathbb{O}}\right)\right. \\
& \left.+\stackrel{\chi}{r}_{\text {mort } T} e^{-\frac{T}{T_{\text {cold }}}}\right) \stackrel{\chi}{Y^{\prime}} \mathbb{C}, \mathbb{N}, \mathbb{P} .
\end{aligned}
$$

Also, zoobenthos types are kept at a fixed stoichiometric quota $\stackrel{\chi}{q}_{\text {ref } f_{\mathbb{N}, \mathbb{C}: \mathbb{C}}}$ according to Eqs. (268) and (269) resulting in the release of nutrients in inorganic form and carbon in the form of degradable organic matter.

\subsection{Organic matter}

The cycling of carbon, nitrogen, and phosphorus through the benthic food web by the processes of uptake, scavenging, excretion, mortality, release, and burial results in the following organic matter fluxes. 
The dissolved organic matter is produced by excretion and mortality and reduced by bacterial uptake

$$
\begin{aligned}
\left.\frac{\partial \stackrel{\text { dis }}{Q}_{\mathbb{C}, \mathbb{N}, \mathbb{P}}}{\partial t}\right|_{\text {dis }} & =\left.\frac{\partial \stackrel{\text { dis }}{Q}_{\mathbb{C}, \mathbb{N}, \mathbb{P}}}{\partial t}\right|_{\text {excr }}+\left.\frac{\partial \stackrel{\text { dis }}{Q}_{\mathbb{C}, \mathbb{N}, \mathbb{P}}}{\partial t}\right|_{\text {mort }} \\
& -\left.\frac{\partial{ }^{\text {dis }} Q_{\mathbb{C}, \mathbb{N}, \mathbb{P}}}{\partial t}\right|_{\text {upt }} .
\end{aligned}
$$

Degradable matter is generated by excretion and mortality and release fluxes, taken up by bacteria, and scavenged by zoobenthos

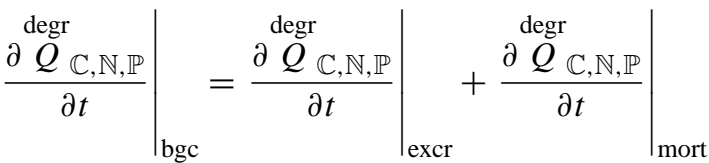

$$
\begin{aligned}
& -\frac{\partial \stackrel{\text { degr }}{Q} \mathbb{C}, \mathbb{N}, \mathbb{P}^{\partial t}}{\left.\right|_{\text {upt }}}-\frac{\partial \stackrel{\text { degr }}{\mathbb{C}, \mathbb{N}, \mathbb{P}}_{\partial t}}{\text { scav }} \\
& {\left[+\left.\frac{\partial \stackrel{\text { degr }}{Q}_{\mathbb{C}}}{\partial t}\right|_{\mathrm{rel}}\right]}
\end{aligned}
$$

Refractory matter is taken up by bacteria and modified by burying across the total depth horizon

$\left.\frac{\partial Q_{\mathbb{C}, \mathbb{N}, \mathbb{P}}^{\text {refr }}}{\partial t}\right|_{\text {bgc }}=-\left.\frac{\partial Q_{\mathbb{C}, \mathbb{N}, \mathbb{P}}^{\text {refr }}}{\partial t}\right|_{\text {upt }}-\left.\frac{\partial Q_{\mathbb{C}, \mathbb{N}, \mathbb{P}}^{\text {refr }}}{\partial t}\right|_{\text {bur }}$.

The abbreviated cycles for iron and silicate condensate all biogeochemical processes in the benthos into a simple remineralization of degradable organic matter into dissolved inorganic iron or silicate at a fixed rate $r_{\mathbb{F r e m i n}}$ or $r_{\text {Sremin }}$ :

$$
\begin{aligned}
& \left.\frac{\partial \stackrel{\text { degr }}{Q_{\mathbb{F}}}}{\partial t}\right|_{\text {bgc }}=-r_{\mathbb{F} \text { remin }} \stackrel{\text { degr }}{Q_{\mathbb{F}}^{\prime}}, \\
& \left.\frac{\partial \stackrel{\text { degr }}{Q_{\mathbb{S}}}}{\partial t}\right|_{\text {bgc }}=-r_{\text {Sremin }} \stackrel{\text { degr }}{Q_{\mathbb{S}}^{\prime}} .
\end{aligned}
$$

In these equations the partitioning in between the different forms of organic matter occurs in the following manner.

Uptake of all forms of organic matter by bacteria is given by Eqs. (163)-(165) as

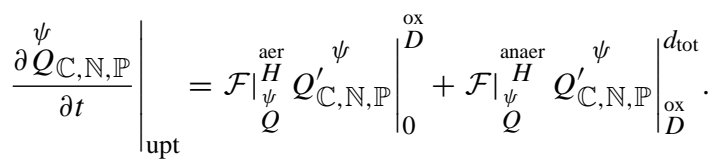

The excretion of aerobic bacteria is directed to dissolved organic matter, while for the zoobenthos types $\stackrel{\Psi}{Y}$ it is directed to degradable matter:

$\frac{\partial \stackrel{\text { dis }}{\mathbb{C}, \mathbb{N}, \mathbb{P}}_{\partial t}}{\partial t}=\left.\frac{\partial \stackrel{H}{\mathbb{C}, \mathbb{N}, \mathbb{P}}_{\text {excr }}^{\text {ar }}}{\partial t}\right|_{\text {excr }}$,
$\left.\frac{\partial \stackrel{\operatorname{degr}}{Q}_{\mathbb{C}, \mathbb{N}, \mathbb{P}}}{\partial t}\right|_{\text {excr }}=\left.\sum_{\Psi} \frac{\partial Y_{\mathbb{C}, \mathbb{N}, \mathbb{P}}}{\partial t}\right|_{\text {excr }}$,

using Eqs. (168), (169), (179), and (180).

The mortality of aerobic bacteria is partitioned between a particulate part directed to degradable matter and a dissolved part $\stackrel{\text { aer }}{q}$ dmort , while for the zoobenthos types $\stackrel{\Psi}{Y}$ and anaerobic bacteria it is entirely directed to degradable matter:

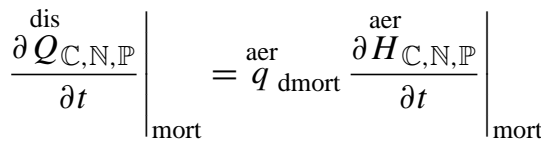

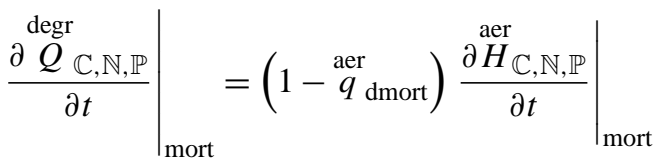

$$
\begin{aligned}
& +\left.\frac{\partial \stackrel{\text { anaer }}{H} \mathbb{C}, \mathbb{N}, \mathbb{P}}{\partial t}\right|_{\text {mort }}+\left.\sum_{\Psi} \frac{\partial \stackrel{\Psi}{Y}_{\mathbb{C}, \mathbb{N}, \mathbb{P}}}{\partial t}\right|_{\text {mort }}
\end{aligned}
$$

using Eqs. (171) and (182).

Degradable matter is scavenged by zoobenthos according to Eq. (177):

$\left.\frac{\partial \stackrel{\text { degr }}{Q}}{\partial t}\right|_{\mathbb{C}, \mathbb{N}, \mathbb{P}}=\left.\sum_{\Psi} \mathcal{F}\right|_{\substack{\text { degr } \\ Q}} ^{\stackrel{\Psi}{Y}} \stackrel{\operatorname{degr}}{Q}_{\mathbb{C}, \mathbb{N}, \mathbb{P}}^{\prime} \cdot$

In addition, degradable carbon may be produced by the stoichiometric adjustment (Eq. 268) of bacteria or zoobenthos:

$\left.\frac{\partial Q_{\mathbb{C}}^{\text {degr }}}{\partial t}\right|_{\text {rel }}=\left.\sum_{\chi} \frac{\partial \stackrel{\chi}{H}_{\mathbb{C}}}{\partial t}\right|_{\text {rel }}+\left.\sum_{\Psi} \frac{\partial Y_{\mathbb{C}}}{\partial t}\right|_{\text {rel }}$.

The diffusive process of bioturbation leads to the downward displacement of refractory material. The resulting flux of refractory organic matter across the total depth horizon of living organisms in the model $d_{\text {tot }}$ may be interpreted as burial flux (activated by the ISWbur switch), as material is removed from the biogeochemical active part of the model.

To derive this flux we use a simple geometric argument here: it is assumed that the diffusive process will preserve the vertically exponential distribution of refractory organic matter (Eq. 154), stretching it. Consequently the flux across any 
horizontal interface can be expressed as the product of the local concentration $\stackrel{\text { refr }}{c} \mathbb{C}, \mathbb{N}, \mathbb{P}$ and the displacement rate of the exponential profile at the given level. Specifically, we know that the local displacement rate at the level of the penetration depth is precisely the change of penetration depth due to bioturbation $\left.\frac{\partial^{\mathrm{refr} C, \mathbb{N}, \mathbb{P}}}{\partial t}\right|_{\text {bturb }}$.

To derive the local displacement rate of the exponential profile at the total depth, we can use the displacement timescale at $d_{\text {tot }}$, that is, independent of the local concentration:

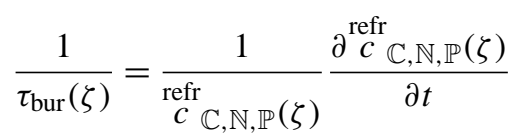

$$
\begin{aligned}
& =\left.\frac{\zeta}{\underset{D}{\operatorname{refr} \mathbb{C}, \mathbb{P}}}{ }^{2} \frac{\partial \operatorname{refr}_{\mathbb{C}, \mathbb{N}, \mathbb{P}}}{\partial t}\right|_{\text {bturb }} .
\end{aligned}
$$

Scaling the displacement rate using this scale, the flux of matter at $d_{\mathrm{tot}}$, and hence the burial flux, can be computed as

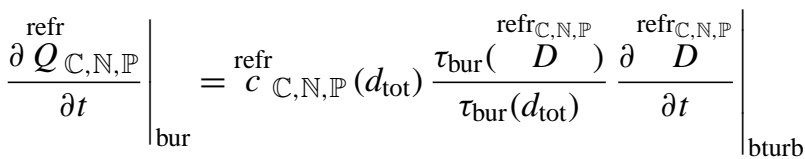

$$
\begin{aligned}
& =\left.\stackrel{\operatorname{refr}}{c} \mathbb{C , N , \mathbb { P }}\left(d_{\mathrm{tot}}\right) \frac{d_{\mathrm{tot}}}{\operatorname{refr}_{\mathbb{C}, \mathbb{N}, \mathbb{P}}} \frac{\partial \operatorname{refr}_{\mathbb{C}, \mathbb{N}, \mathbb{P}}}{\partial t}\right|_{\mathrm{bturb}}
\end{aligned}
$$

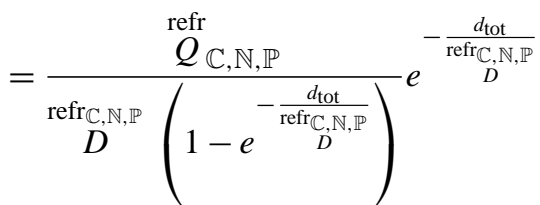

$$
\begin{aligned}
& \left.\frac{d_{\mathrm{tot}}}{\operatorname{refr}_{\mathbb{C}, \mathbb{N}, \mathbb{P}}} \frac{\partial^{\operatorname{refr}} D}{\partial t}\right|_{\text {bturb }} .
\end{aligned}
$$

This result can be formally confirmed by a straightforward but fairly lengthy derivation of the time derivative of the integrated content of refractory matter between the sediment surface and $d_{\text {tot }}$ using Eqs. (154) and (160).

Note that this process removes biomass from the biogeochemically active part of the model, as there are no processes connected to buried organic matter and the model currently does not consider remobilization. This means that during long-term simulations the loss of nutrients needs to be compensated for, e.g. by riverine inputs or atmospheric deposition (carbon is restored by air-sea exchange).

\subsection{Inorganic components}

The dynamics of benthic nutrients are given by the following equations (see Eq. 187 for the remineralization of silicate):

$$
\begin{aligned}
& \left.\frac{\partial K_{\mathbb{N}}^{\mathrm{x}}}{\partial t}\right|_{\mathrm{bgc}}=\left.\frac{\partial K_{\mathbb{N}}^{\mathrm{ox}}}{\partial t}\right|_{\text {nitr }}-\left.\frac{\partial K_{\mathbb{N}}}{\partial t}\right|_{\text {denit }}, \\
& \left.\frac{\partial K_{\mathbb{N}}}{\partial t}\right|_{\text {bgc }}=-\left.\frac{\partial K_{\mathbb{N}}}{\partial t}\right|_{\text {nitr }}-\left.\frac{\partial K_{\mathbb{N}}}{\partial t}\right|_{\text {upt }}+\left.\frac{\partial K_{\mathbb{N}}}{\partial t}\right|_{\text {rel }},
\end{aligned}
$$

$$
\begin{aligned}
& \left.\frac{\partial K_{\mathbb{P}}}{\partial t}\right|_{\mathrm{bgc}}=-\left.\frac{\partial K_{\mathbb{P}}}{\partial t}\right|_{\mathrm{upt}}+\left.\frac{\partial K_{\mathbb{P}}}{\partial t}\right|_{\text {rel }}, \\
& \left.\frac{\partial K_{\mathbb{S}}}{\partial t}\right|_{\mathrm{bgc}}=r_{\text {Sremin }} Q_{\mathbb{S}}^{\prime},
\end{aligned}
$$

while the biogeochemistry of dissolved carbon, oxygen and dinitrogen are given by

$$
\begin{aligned}
& \left.\frac{\partial G_{\mathbb{C}}}{\partial t}\right|_{\mathrm{bgc}}=\left.\frac{\partial G_{\mathbb{C}}}{\partial t}\right|_{\text {resp }} \\
& \left.\frac{\partial G_{\mathbb{O}}}{\partial t}\right|_{\mathrm{bgc}}=-\left.\frac{\partial G_{\mathbb{O}}}{\partial t}\right|_{\text {resp }}-\left.\frac{\partial G_{\mathbb{O}}}{\partial t}\right|_{\text {nitr }} \\
& \left.\frac{\partial G_{\mathbb{N}}}{\partial t}\right|_{\mathrm{bgc}}=\left.\frac{\partial G_{\mathbb{N}}}{\partial t}\right|_{\text {denit }} .
\end{aligned}
$$

The respiration terms of dissolved inorganic carbon and dissolved oxygen are given by Eqs. (170) and (181) as

$$
\begin{aligned}
& \left.\frac{\partial G_{\mathbb{C}}}{\partial t}\right|_{\text {resp }}=\left.\sum_{\chi} \frac{\partial \stackrel{\chi}{H_{\mathbb{C}}}}{\partial t}\right|_{\text {resp }}+\left.\sum_{\Psi} \frac{\partial Y_{\mathbb{C}}}{\partial t}\right|_{\text {resp }}, \\
& \left.\frac{\partial G_{\mathbb{O}}}{\partial t}\right|_{\text {resp }}=-q_{\mathbb{O}: \mathbb{C}}\left(\left.\frac{\partial \stackrel{\text { aer }}{\mathbb{C}}}{\partial t}\right|_{\text {resp }}+\left.\sum_{\Psi} \frac{\partial Y_{\mathbb{C}}}{\partial t}\right|_{\text {resp }}\right),
\end{aligned}
$$

where $q_{\mathbb{O}}: \mathbb{C}$ is the oxygen to carbon conversion coefficient.

Nitrification in the benthos is computed similarly to the pelagic nitrification from a maximum ammonium massspecific nitrification rate ${ }_{r}^{H}$ nitr at reference temperature, depending on the ammonium available in the oxygenated layer, approximated as $\frac{\stackrel{D}{D}}{d_{\mathrm{tot}}} K_{\mathbb{N}}^{\prime}$ :

$$
\left.\frac{\partial \stackrel{\text { ox }}{K_{\mathbb{N}}}}{\partial t}\right|_{\text {nitr }}=\left.\frac{\partial K_{\mathbb{N}}^{\text {amm }}}{\partial t}\right|_{\text {nitr }}=r_{\text {nitr }}^{H} \quad \begin{array}{llll}
l_{T} & l_{\mathbb{N}} & \frac{D}{d_{\text {tot }}} & K^{\prime}
\end{array}
$$


$\left.\frac{\partial G_{\mathbb{O}}}{\partial t}\right|_{\text {nitr }}=\left.2 \frac{\partial K_{\mathbb{N}}^{\text {ox }}}{\partial t}\right|_{\text {nitr }}$,

where $l_{\mathbb{N}}$ and $l_{T}$ bnitr are the nitrification limitation factors due to the presence of high concentrations of oxidized nitrogen and the temperature regulation factor (Eqs. 257 and 239).

Denitrification is calculated from the oxidized nitrogen reduction equivalent required for anaerobic bacteria respiration:

$$
\begin{gathered}
\stackrel{\mathcal{F}_{\text {req }}^{\text {anaer }}=}{ }=\frac{1}{2\left(1-\stackrel{H}{q}_{\text {denit }}\right)+\frac{5}{4} \stackrel{H}{q}_{\text {denit }}} \\
{\left.\stackrel{H}{\text { red }} q_{\mathbb{O}: \mathbb{C}} \frac{\partial \stackrel{H}{H}_{\mathbb{C}}}{\partial t}\right|_{\text {resp }}}^{,}
\end{gathered}
$$

where $\stackrel{H}{q}_{\text {red }}$ is the maximum fraction of anaerobic bacteria respiration resulting in oxidized nitrogen reduction, $\stackrel{H}{q}$ denit is the fraction of reduction subject to denitrification as opposed to ammonification, and $2, \frac{5}{4}$ are the stoichiometric coefficients of oxygen demand per reduction equivalent for the ammonification and denitrification reactions respectively.

The actual reduction of oxidized nitrogen by denitrification is then further limited by availability of oxidized nitrogen $\left(l_{\mathbb{N}}^{\text {denit }}\right.$, Eq. 258$)$, resulting in the following denitrification fluxes:

$$
\begin{aligned}
& \left.\frac{\partial K_{\mathbb{N}}^{\text {ox }}}{\partial t}\right|_{\text {denit }}=l_{\mathbb{N}} \quad \mathcal{F}_{\text {req }}, \\
& \left.\frac{\partial K_{\mathbb{N}}^{\text {denit }}}{\partial t}\right|_{\text {denit }}=\left.\left(1-q_{\operatorname{red} N_{2}}\right) \frac{\partial \mathbb{K}_{\mathbb{N}}^{\text {ax }}}{\partial t}\right|_{\text {denit }}, \\
& \left.\frac{\partial G_{\mathbb{N}}}{\partial t}\right|_{\text {bgc }}=\left.q_{\text {red } G} \frac{\partial K_{\mathbb{N}}^{\text {ox }}}{\partial t}\right|_{\text {denit }},
\end{aligned}
$$

where $q_{\mathrm{red} G}$ is the fraction of reduction directed to dinitrogen. As nitrogen fixation is currently not considered in the model, losses of oxidized nitrogen by denitrification are removed from the active cycle and need to be compensated for in long-term runs by riverine or atmospheric inputs; otherwise, denitrification needs to be switched off.

Release of nutrients caused by stoichiometric adjustment (Eq. 268) of bacteria or zoobenthos is given by

$$
\begin{aligned}
& \left.\frac{\partial K_{\mathbb{N}}^{\mathrm{amm}}}{\partial t}\right|_{\text {rel }}=\sum_{\chi} \frac{\partial{\stackrel{\chi}{H_{\mathbb{N}}}}_{\partial t}}{\left.\right|_{\text {rel }}}+\left.\sum_{\Psi} \frac{\partial Y_{\mathbb{N}}^{\Psi}}{\partial t}\right|_{\text {rel }}, \\
& \left.\frac{\partial K_{\mathbb{P}}}{\partial t}\right|_{\text {rel }}=\left.\sum_{\chi} \frac{\partial \stackrel{\chi}{H}_{\mathbb{P}}}{\partial t}\right|_{\text {rel }}+\left.\sum_{\Psi} \frac{\partial Y_{\mathbb{P}}}{\partial t}\right|_{\text {rel }} .
\end{aligned}
$$

\subsection{Bioirrigation}

The diffusivity of dissolved inorganic states is given by a basal diffusivity $\vartheta_{\chi}$ for each layer $\chi$ : aer, den, anox that is increased for bioirrigation by the factor $p_{\text {bimin }}$. The activity of deposit feeders and meiofauna cause further enhancement to yield the total bioirrigation diffusivity $v_{\text {idiff }}$ (used in Eq. 145):

$\mathcal{S}_{\text {birr }}=\left.{ }_{q}^{\mathrm{DEPO}}{ }_{\text {birr }} \frac{\partial Y_{\mathbb{C}}^{\mathrm{DEPO}}}{\partial t}\right|_{\text {upt }}+{ }_{q}^{\mathrm{MEIO}}$ birr $\left.\frac{\partial Y_{\mathbb{C}}}{\partial t}\right|_{\text {upt }}$
$\nu_{\text {idiff }}=\vartheta_{\chi}\left(p_{\text {bimin }}+p_{\text {bienh }} \frac{\mathcal{S}_{\text {birr }}}{\mathcal{S}_{\text {birr }}+h_{\text {birr }}}\right)$,

where $\stackrel{\text { DEPO }}{q}$ birr and ${ }_{q}^{\text {MEIO }}$ birr are the fractions of deposit feeder and meiobenthos uptake contributing to bioirrigation, $h_{\text {birr }}$ is a half-saturation rate for bioirrigation enhancement and $p_{\text {bienh }}$ is the maximum bioturbation enhancement factor of dissolved inorganic diffusion in the benthos.

\subsection{Bioturbation}

For particulate matter in the benthos sediment diffusion $v_{\text {bturb }}$ in Eq. (158) is based on a background diffusivity $\vartheta_{\text {part }}$ and an enhancement factor of Michaelis-Menten type depending on the bioturbation caused by deposit feeder activity (see Eq. 178):

$\mathcal{S}_{\text {bturb }}=\stackrel{\text { DEPO }}{q}$ bturb $\left._{\frac{\partial Y_{\mathbb{C}}}{\partial t}}\right|_{\text {upt }}$
$\nu_{\text {bturb }}=\vartheta_{\text {part }}\left(1+p_{\text {btenh }} \frac{\mathcal{S}_{\text {bturb }}}{\mathcal{S}_{\text {bturb }}+h_{\text {bturb }}}\right)$,

where $\stackrel{\text { DEPO }}{q}$ bturb is the fraction of deposit feeder uptake contributing to bioturbation, $h_{\text {bturb }}$ is a half-saturation rate for bioturbation enhancement and $p_{\text {btenh }}$ is the maximum bioturbation enhancement factor of particulate matter diffusion in the benthos.

\section{Horizontal interfaces}

\subsection{The benthic-pelagic interface}

The boundary condition at the seabed is given by the deposition of sinking particulate organic material, phytoplankton, and calcite on the seafloor, the diffusion of inorganic chemical components between the porewater and the pelagic water column, and resuspension of organic matter. All other state variables generally have no flux conditions at the pelagicbenthic interface. 


\subsubsection{Deposition of organic matter and phytoplankton}

Deposition fluxes are taken analogous to the gravitational sinking rates in Eq. (143) where the sinking velocity is replaced by the deposition velocity $\underset{w_{c_{p}}}{\text { deccording to the seabed }}$ shear stress $\tau_{\text {bed }}$ :

$\stackrel{c}{\mathrm{p}}_{w_{\text {depo }}}=\max \left(1-\frac{\tau_{\text {bed }}}{\tau_{\text {crit }}}, 0\right) \stackrel{c_{\mathrm{p}}}{w_{\text {sed }}}$,

leading to the deposition fluxes

$\left.\mathcal{F}\right|_{c_{\mathrm{p}}} ^{\text {ben }}={\stackrel{c_{\mathrm{p}}}{w}}_{\mathrm{depo}} c_{\mathrm{p}}^{\prime}$

As for gravitational sinking, the only state variables sedimenting onto the seafloor are particulate organic matter, the phytoplankton components, and calcite $\left(\stackrel{\Psi}{M}_{\mathbb{C}, \mathbb{N}, \mathbb{P}, \mathbb{F}, \mathbb{S}}\right.$, $\stackrel{\chi}{P}_{\mathbb{C}, \mathbb{N}, \mathbb{P}, \mathbb{F}, \mathbb{S}, \mathcal{C}}$, and $\stackrel{\text { calc }}{L}_{\mathbb{C}}$ ). The absorption of deposited carbon, nitrogen, and phosphorus components into the sediments then results in separation of the organic material into dissolved, degradable, and refractory matter according to

$$
\begin{aligned}
& \left.\mathcal{F}\right|_{\text {pel }} ^{\stackrel{\text { degr }}{Q_{\mathbb{C}}}}=\left.\left(1-\stackrel{\chi}{q}_{\text {ddepo }}-\stackrel{\chi}{q}_{\text {rdepo }}\right) \sum_{\chi} \mathcal{F}\right|_{P_{\mathbb{C}}} ^{\text {ben }} \\
& +\left.\stackrel{\text { part }}{q \text { rdepo }}_{\chi \chi} \mathcal{F}\right|_{R_{\mathbb{C}}} ^{\text {ben }},
\end{aligned}
$$

$\left.\mathcal{F}\right|_{\text {pel }} ^{\stackrel{\text { dis }}{Q}=\stackrel{\chi}{q}}=\left.\stackrel{\text { ddepo }}{\text { de }}_{\chi} \sum_{\chi} \mathcal{F}\right|_{P_{\mathbb{C}}^{\chi}} ^{\text {ben }}$,

where $\stackrel{\chi}{q}_{\text {ddepo }}$ and $\stackrel{\chi}{q}_{\text {rdepo }}$ are the dissolved and refractory fractions of deposing material. For nitrogen and phosphorus the portioning is modified according to the relative cytoplasm

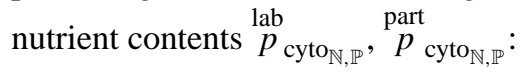

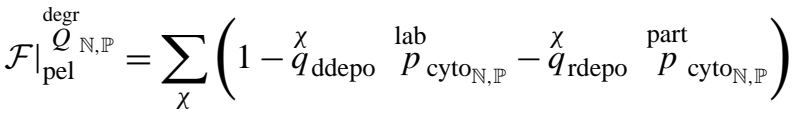

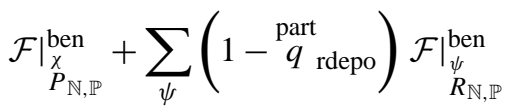

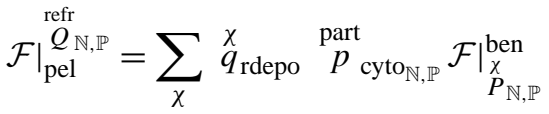

$$
\begin{aligned}
& +\left.\sum_{\psi} \underset{q \text { rdepo }}{\text { part }} \mathcal{F}\right|_{R_{\mathbb{N}, \mathbb{P}}} ^{\text {ben }}
\end{aligned}
$$

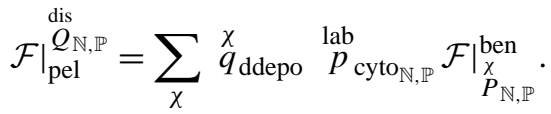

The iron and silicate components are entirely directed to degradable matter, the only state considered for these components in the benthic model:

$$
\begin{aligned}
& \left.\mathcal{F}\right|_{\text {pel }} ^{Q_{\mathbb{F}}^{\text {degr }}}=\left.\sum_{\chi} \mathcal{F}\right|_{P_{\mathbb{F}}^{\chi}} ^{\text {ben }}+\left.\mathcal{F}\right|_{\substack{\text { small } \\
R_{\mathbb{F}}}} ^{\text {ben }}+\left.\mathcal{F}\right|_{\substack{\text { med } \\
R_{\mathbb{F}}}} ^{\text {ben }}, \\
& \left.\mathcal{F}\right|_{\text {pel }} ^{Q_{\mathbb{S}}^{\text {degr }}}=\left.\mathcal{F}\right|_{P_{\mathbb{S}}^{\text {dia }}} ^{\text {ben }}+\left.\mathcal{F}\right|_{\substack{\text { med } \\
R_{\mathbb{S}}}} ^{\text {ben }}+\left.\mathcal{F}\right|_{\substack{\text { large } \\
R_{\mathbb{S}}}} ^{\text {ben }} .
\end{aligned}
$$

Calcite deposition is given by

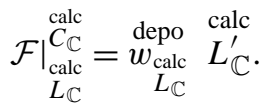

\subsubsection{Resuspension}

In the case of strong shear stress $\tau_{\text {bed }}$ at the seafloor part of the sediments may get resuspended into the water column. The erosion flux is calculated proportional to the excess shear stress over a critical threshold $\tau_{\text {crit }}$ by a reference erosion flux $r_{\mathrm{er}}$. Erosion in terms of particulate organic matter is then approximated as a fraction of the total sediment matter ${ }_{Q}^{\text {sed }}+\stackrel{\text { degr }}{Q_{\mathbb{C}}}$ :

$$
\begin{aligned}
& \stackrel{\mathcal{S}}{\text { resusp }}=\frac{r_{\text {er }} \max \left(\frac{\tau_{\text {bed }}}{\tau_{\text {crit }}}-1,0\right)}{p_{Q}+\stackrel{\text { segr }}{Q}_{\mathbb{C}}} \\
& \left.\mathcal{F}_{\mathbb{C}, \mathbb{N}, \mathbb{P}, \mathbb{F}, \mathbb{S}}^{\text {resusp }}\right|_{Q} ^{\substack{\text { med } \\
R}}=\stackrel{\mathcal{S}}{\text { resusp }} \stackrel{\text { degr }}{Q} \mathbb{C}, \mathbb{N}, \mathbb{P}, \mathbb{F}, \mathbb{S} .
\end{aligned}
$$

The values and approximations used for the three parameters $\tau_{\text {crit }}, r_{\text {er }}$ and ${ }_{p}^{\text {sed }}$ are given in the Supplement.

\subsubsection{Inorganic fluxes across the seabed}

The diffusion of dissolved inorganic states across the benthos is derived from the equilibrium conditions described in Sect. 4.2. Based on the tendency of the system towards equilibrium, the total flux across the seabed is then given by the sum of all sources and sinks and a relaxation towards equilibrium:

$$
-\stackrel{\chi \mathcal{F}}{\text { ben }}=\left.\frac{\partial \chi}{\partial t}\right|_{\text {bgc }}+\frac{1}{\tau_{\text {eq }}}\left(\chi-p_{\text {poro }} p_{\text {Cads }} \tilde{\chi}_{\text {pw }}\right),
$$


where $\chi$ represents the inorganic states of oxygen, DIC, oxidized nitrogen, ammonium, phosphate, and silicate.

For phosphorus, ammonium, silicate, and DIC, the relaxation fluxes towards equilibrium are computed by assuming a parabolic vertical distribution of excess biomass with 0 surface concentration and 0 bottom flux and assuming contributions to the generation of the excess proportional to the layer depth. The compensation flux across the seabed is then again computed from the production-diffusion balance in Eq. (145). For oxidized nitrogen and oxygen the procedure requires modification for two reasons: the separation depths of the oxygenated layer and denitrification layer given by the dissolved oxygen horizon and the horizon of oxidized nitrogen may be considered as fixed parameters for the diffusionproduction balance of the other state variables, but not so for dissolved oxygen and oxidized nitrogen, whose biogeochemical changes affect the dynamics of these horizons directly. In addition, the system imposes a third boundary condition on the balance equation, i.e. that the concentration at the respective horizon has to be zero by definition (and no sources and sinks exist below these limits), which renders the system overdetermined. For these two variables the relaxation timescale is therefore approximated by the fixed parameters $\tau_{\mathrm{ox}}$ and $\tau_{\text {denit }}$ also used to determine the dynamical evolution of oxygen and the oxidized nitrogen horizon in Eqs. (148) and (149).

The recycling of iron in the benthos is abbreviated, as there is very little information on the iron cycle in the seabed. The only form of iron considered in the benthos is the degradable matter, which is implicitly remineralized and returned to the water column in dissolved form at a fixed remineralization rate $\stackrel{\chi}{r}_{\text {remin }}$ :

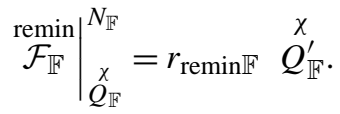

\subsubsection{Remineralization of calcite}

No processes related to the formation or dissolution of calcite in the benthos are currently included in the model; the benthic cycle of calcite is resolved purely implicitly, similar to iron, as a simple linear release to the water column of the calcite deposited onto the sediments:

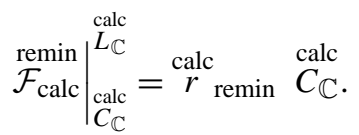

\subsubsection{Benthic remineralization sub-model}

As an alternative to the full benthic model described in the Sect. 4, a simple benthic closure is available that implicitly remineralizes benthic substrate into dissolved inorganic states, analogous to the treatment of iron and calcite above. The treatment of deposition and resuspension of organic matter on the seafloor in this case is identical to the full benthic model, while the recycling of organic matter occurs as a linear function of the benthic content at a given remineralization rate $\stackrel{\chi}{r}_{\text {remin }}$ :

$\underset{\mathcal{F}}{\operatorname{remin}} \underset{\mathbb{C}, \mathbb{P}, \mathbb{S}}{\left.\right|_{Q_{\mathbb{C}, \mathbb{P}, \mathbb{S}}} ^{\sigma_{\mathbb{C}}, N_{\mathbb{P}}, N_{\mathbb{S}}}}=\stackrel{\chi}{r}_{\text {remin }} \stackrel{\stackrel{\chi}{Q}^{\prime}}{\mathbb{C}, \mathbb{P}, \mathbb{S}}$.

For nitrogen the remineralization flux is split, regenerating oxidized nitrogen and ammonium using the fixed fraction $\stackrel{\chi}{q}_{\text {remin }}$ :

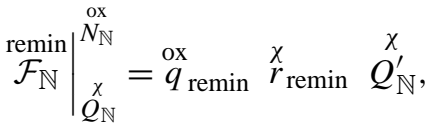

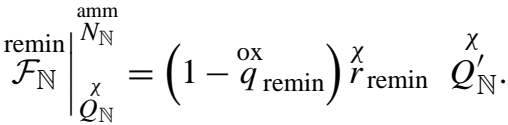

With this option no other biogeochemical processes are considered in the benthos. The treatment of iron and calcite is identical between the full benthic model and this simplified benthic closure.

\subsection{Sea-surface fluxes}

The only two boundary fluxes computed in the standard setup at the air-sea interface are the exchange of oxygen and carbon dioxide. Other processes like atmospheric deposition of nutrients and riverine inputs require spatially varying surface fields and are best provided through the physical driver. (Implementations of this type have been used in Artioli et al., 2012; Edwards et al., 2012; Holt et al., 2012; Wakelin et al., 2012.)

Oxygen is exchanged based on the difference from the saturation state, which is estimated according to Weiss (1970):

$\left.\mathcal{F}_{\mathbb{O}}\right|_{\text {sea }} ^{\text {air }}=k_{\text {air } \mathbb{O}}\left(T, S, \boldsymbol{u}_{\text {wind }}\right)\left(O_{\mathbb{O}}-s_{\mathbb{O}}\right)$.

The regression formula for $s_{\mathbb{O}}$ is given in the Supplement.

The exchange of carbon dioxide is based on the difference in partial pressures

$\left.\mathcal{F}_{\mathbb{C}}\right|_{\text {sea }} ^{\text {air }}=\rho_{\text {sea }} k_{\text {air } \mathbb{C}}\left(T, \boldsymbol{u}_{\text {wind }}\right)\left(\mathrm{p}_{\mathrm{CO}_{2}}-\underset{\mathrm{P}_{\mathrm{CO}_{2}}}{\text { air }}\right)$,

where pair $\mathrm{CO}_{2}$ maybe be provided by the physical driver or a constant parameter $\stackrel{\text { air }}{\mathrm{CO}_{2}}$.

The empirical gas transfer coefficients $k_{\text {air } \mathbb{O}}$ and $k_{\text {air } \mathbb{C}}$ are taken from Weiss (1970) and Nightingale et al. (2000) and given in the Supplement.

\section{Generic terms}

\subsection{Regulation and limitation factors}

The regulation of metabolic processes by temperature is modelled using the $Q_{10}$ function introduced in Blackford 
et al. (2004) that strongly increases at low temperatures and decreases slower at high temperatures representing enzyme degradation:

$\stackrel{\chi}{l_{T}}=\stackrel{\chi}{p}_{Q_{10}} \frac{T\left[^{\circ} \mathrm{Cl}-10^{\circ} \mathrm{C}\right.}{10^{\circ} \mathrm{C}}-\stackrel{\chi}{p}_{Q_{10}} \frac{\frac{\left.T{ }^{\circ} \mathrm{C}\right]-32^{\circ} \mathrm{C}}{3^{\circ} \mathrm{C}}}{,}$

where $T\left[{ }^{\circ} \mathrm{C}\right]$ is the water temperature in degrees Celsius and $\chi$ represents the respective process or state.

Nitrogen and phosphorus limitation factors for each of the four phytoplankton types are based on Droop kinetics (Droop, 1974) and computed as

$$
\begin{aligned}
& \stackrel{\chi}{l}_{\mathbb{P}}=\min \left(1, \max \left(0, \frac{\stackrel{\chi}{q}_{\mathbb{P}: \mathbb{C}}-\stackrel{\chi}{q}_{\min \mathbb{P}: \mathbb{C}}}{\chi_{\mathrm{ref}_{\mathbb{P}: \mathbb{C}}}-\stackrel{\chi}{q}_{\text {min } \mathbb{P}: \mathbb{C}}}\right)\right), \\
& \stackrel{\chi}{l}_{\mathbb{N}}=\min \left(1, \max \left(0, \frac{\stackrel{\chi}{q}_{\mathbb{N}: \mathbb{C}}-\stackrel{\chi}{q}_{\text {min }_{\mathbb{N}: \mathbb{C}}}}{\chi_{\mathrm{q}_{\mathrm{ref}}: \mathbb{C}}-\stackrel{\chi}{q}_{\min _{\mathbb{N}: \mathbb{C}}}}\right)\right),
\end{aligned}
$$

where $\chi$ represents any phytoplankton type (dia, micro, nano, pico), $\stackrel{\chi}{q}_{\text {ref }_{\mathrm{N}, \mathbb{P}: \mathbb{C}}}$ is its reference internal quota and $\stackrel{\chi}{q}_{\text {min } \mathbb{N}, \mathbb{P}: \mathbb{C}}$ is its minimal internal quota. These two factors are combined to three alternative forms of co-limitation $\stackrel{x}{l}_{\langle\mathbb{N} P}$

$\stackrel{x}{l}_{\langle\mathbb{N} \mathbb{P}\rangle}=f\left(\begin{array}{ll}\chi & \chi \\ l_{\mathbb{N}} & ,\end{array}\right.$

switchable through the namelist switch LimnutX:

Limnutx $=0 ; \stackrel{\chi}{l}_{\langle\mathbb{N P P}\rangle}$ is the geometric mean of $\stackrel{\chi}{l}{ }_{\mathbb{N}}$ and $\stackrel{\chi}{l}_{\mathbb{P}}$,

Limnutx $=2: \stackrel{\chi}{l}_{\langle\mathbb{N P}\rangle}$ is the harmonic mean of $\stackrel{\chi}{l}_{\mathbb{N}}$ and $\stackrel{\chi}{l}_{\mathbb{P}}$,

Limnutx $=1 ; \stackrel{\chi}{l}_{\langle\mathbb{N} \mathbb{P}\rangle}$ is the minimum of $\stackrel{\chi}{l}_{\mathbb{N}}$ and $\stackrel{\chi}{l}_{\mathbb{P}}$.

The silicate limitation factor for diatoms is computed from the external availability of dissolved silicate $N_{\mathbb{S}}$, based on a Michaelis-Menten term with half-saturation $\frac{\mathrm{dia}}{h_{\mathbb{S}}}$ :

$$
\stackrel{\text { dia }}{l_{\mathbb{S}}}=\frac{N_{\mathbb{S}}}{N_{\mathbb{S}}+{ }_{h_{\mathbb{S}}}^{\text {dia }}} .
$$

The iron limitation factor is computed in the same way as the factors for nitrogen and phosphorus:

$\stackrel{\chi}{l}_{\mathbb{F}}=\min \left(1, \max \left(0, \frac{\stackrel{\chi}{q}_{\mathbb{F}: \mathbb{C}}-\stackrel{\chi}{q}_{\min _{\mathbb{F}: \mathbb{C}}}}{\stackrel{x}{q}_{\mathrm{ref}_{\mathbb{F}: \mathbb{C}}}-\stackrel{\sim}{q}_{\min _{\mathbb{F}: \mathbb{C}}}}\right)\right)$,

with $\stackrel{\chi}{q}_{\text {ref }: \mathbb{C}}$ as its reference internal quota and $\stackrel{\chi}{q}_{\text {min }_{\mathbb{F}: \mathbb{C}}}$ as its minimal internal quota.

$$
B \quad B
$$

Phosphorus and nitrogen limitation $l_{\mathbb{P}}, l_{\mathbb{N}}$ for the standard model of bacteria mediated decomposition can be based on the availability of the resource in dissolved inorganic form (ISWBlimX =1) and substrate or only in inorganic form (ISWBlimX $=2)$ :

$$
l_{\mathbb{P}}^{B}= \begin{cases}\min \left(\frac{N_{\mathbb{P}}}{N_{\mathbb{P}}+h_{\mathbb{P}}}, \frac{{ }_{R_{\mathbb{P}}}}{\operatorname{dis}_{\mathbb{P}}+h_{\mathbb{P}}}\right) & \text { if ISWBIimX }=1 \\ \frac{N_{\mathbb{P}}+R_{\mathbb{P}}}{\text { dis }_{\mathbb{B}}} & \text { if ISWBlimX }=2 \\ N_{\mathbb{P}}+R_{\mathbb{P}}+h_{\mathbb{P}} & \end{cases}
$$

and analogous:

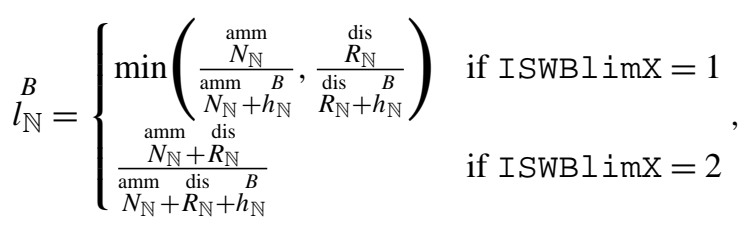

where $h_{\mathbb{P}, \mathbb{N}}$ are the half-saturation constants for phosphorus and nitrogen limitation.

Nutrient regulation of benthic bacteria occurs based on the nutritional state of the substrate

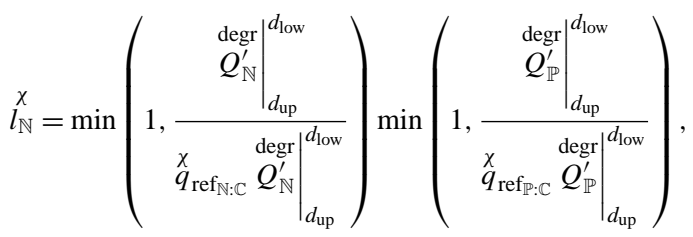

where $\chi$ are aerobic and anaerobic bacteria within the layers described in Sect. 4.4.

Oxygen limitation of zooplankton ( $\chi$ : HET, MICRO, MESO) is computed as function of the relative oxygen saturation state

$s_{\text {relO }}=\min \left(1, \frac{G_{\mathbb{O}}}{s_{\mathbb{O}}}\right)$

$\stackrel{\chi}{l_{\mathbb{O}}}=\frac{s_{\mathrm{rel} \mathbb{O}}+s_{\mathrm{rel} \mathbb{O}} \stackrel{\chi}{h_{\mathbb{O}}}}{s_{\mathrm{rel} \mathbb{O}}+h_{\mathbb{O}}}$,

where the oxygen saturation concentration $s_{\mathbb{O}}$ is estimated according to Weiss (1970). (The regression formula used is given in the Supplement.)

For zoobenthos ( $\chi$ : DEPO, SUSP, MEIO) it is given by a cubic Michaelis-Menten response to the oxygen concentration in the overlying water body in relation to a minimum oxygen threshold $\stackrel{\chi}{p}_{\mathbb{O} \min }$ for each species:

$\stackrel{x}{l_{\mathbb{O}}}=\frac{\max \left(G_{\mathbb{O}}-\stackrel{\chi}{p}_{\mathbb{O} \min }, 0\right)^{3}}{\max \left(G_{\mathbb{O}}-\stackrel{\chi}{p}_{\mathbb{O} \min }, 0\right)^{3}+\stackrel{x}{\mathbb{O}}^{3}}$.

For pelagic bacteria it is given by a simple MichaelisMenten term of the relative oxygen saturation state (Eq. 248)

$\stackrel{B}{l_{\mathbb{O}}}=\frac{s_{\mathrm{rel} \mathbb{O}}}{s_{\mathrm{rel} \mathbb{O}}+h_{\mathbb{O}}}$. 
For benthic bacteria, oxygen regulation occurs through the oxygen and oxidized nitrogen horizons

$$
\stackrel{\text { aer }}{l_{\mathbb{O}}}=\frac{\stackrel{\text { oxy }}{D}}{\stackrel{\text { oxy }}{D+d_{\text {ref }}}}, \quad \stackrel{\text { anaer }}{l_{\mathbb{O}}}=\frac{\stackrel{\text { denit }}{D}-\stackrel{\text { oxy }}{D}}{\stackrel{\text { denit }}{D}-\stackrel{\text { oxy }}{D}+d_{\text {refit }}},
$$

where $\stackrel{\text { oxy }}{d_{\text {ref }}}$ is the aerobic half-saturation depth and $\stackrel{\text { denit }}{d_{\text {ref }}}$ the oxidized half-saturation depth for oxygen regulation.

$l_{\mathbb{O}}^{\text {nitr }}$ is the oxygen limitation factor for nitrification:

${ }_{\text {nitr }}^{l_{\mathbb{O}}}=\frac{O_{\mathbb{O}}^{3}}{O_{\mathbb{O}}^{3}+h_{\mathbb{O}}^{\text {nitr }}}$,

with $h_{\mathbb{O}}$ being the cubic half-saturation constant for oxygen limitation of nitrification.

${ }_{l_{\mathbb{N}}}^{\text {nitr }}$ is the substrate limitation factor for nitrification:

$l_{\mathbb{N}}=\frac{N_{\mathbb{N}}{ }^{\mathrm{amm}^{3}}}{\mathrm{amm}^{3}+\text { nitr }_{\mathbb{N}}}$,

with $h_{\mathbb{N} \text { nitr }}$ being the cubic half-saturation constant for substrate limitation of nitrification and $l_{\mathrm{pH}}$ is the $\mathrm{pH}$-limitation factor for nitrification:

$l_{\mathrm{pH}}=\min (2, \max (0,0.6111 \mathrm{pH}-3.8889))$.

Benthic nitrification is inhibited at a high benthic content of oxidized nitrogen according to

$$
\begin{aligned}
& \overline{K_{\mathbb{N}}}=\frac{\stackrel{\text { ox }}{K_{\mathbb{N}}}}{\frac{\text { oxy } \operatorname{denit} \text { oxy }}{D+\frac{D-D}{3}}},
\end{aligned}
$$

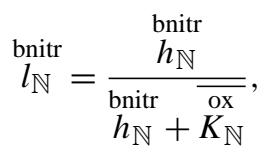

where $h_{\mathbb{N}}$ is the oxygenated layer concentration of oxidized nitrogen at which nitrification is inhibited by $50 \%$.

Here, it is assumed that some oxidized nitrogen penetrates into the denitrification layer, so that the oxygenated layer concentration is on average 3 times higher compared to the denitrification layer.

Based on the same assumption, denitrification in the oxidized layer uses a Michaelis-Menten response to the assumed layer content of oxidized nitrogen:

$$
\begin{aligned}
& \stackrel{\overline{\text { denitr }}}{K_{\mathbb{N}}}=\frac{1}{3} \frac{K_{\mathbb{N}}^{\prime}}{\frac{K_{\text {oxy }}^{\prime}}{D+\frac{\text { denit ox }}{D-D}}} \\
& l_{\mathbb{N}}^{\text {denitr }}=\frac{\overline{K_{\mathbb{N}}}}{\overline{\text { denitr }_{\text {denitr }}}},
\end{aligned}
$$

denitr

where $h_{\mathbb{N}}$ is a denitrification half-saturation constant.

Calcification and dissolution of calcite occur in relation to the calcite saturation state of the water $\Omega_{\text {calc }} \gtrless 1$ (Eq. 131). The regulating factor of the rain ratio for calcification and the regulation factor for dissolution of calcite can be calculated in two alternative ways chosen by the ISWCAL $=1$ namelist switch. The first option (ISWCAL $=1$ ) is based on an exponential term:

$\stackrel{\text { calc }}{l_{\mathbb{C}}}=\max \left(0,\left(\Omega_{\text {calc }}-1\right)^{n_{\text {calc }}}\right)$

$l_{\mathbb{C}}^{\text {dis }}=\max \left(0,\left(1-\Omega_{\text {calc }}\right)^{n_{\mathrm{dis}}}\right)$,

where $n_{\text {calc,dis }}$ are calcification/dissolution exponents (Ridgwell et al., 2007; Keir, 1980).

The second option (ISWCAL $=2$ ) uses a MichaelisMenten term:

$$
\begin{aligned}
& l_{\mathbb{C}}^{\text {calc }}=\max \left(0, \frac{\Omega_{\text {calc }}-1}{\Omega_{\text {calc }}-1+h_{\text {calc }}}\right) \\
& \operatorname{lis}_{\mathbb{C}}=\max \left(0, \frac{1-\Omega_{\text {calc }}}{1-\Omega_{\text {calc }}+h_{\text {calc }}}\right)
\end{aligned}
$$

where $h_{\text {calc }}$ is the half-saturation constant for calcification and dissolution of calcite (Blackford et al., 2010; Gehlen et al., 2007).

The rain ratio (Eq. 94) is regulated by nutrient limitation and temperature to reflect the dependency of the calcifying fraction of nanophytoplankton on the environmental conditions. Temperature regulation is given by

$$
\stackrel{\text { calc }}{\boldsymbol{l}_{T}}=\frac{\max \left(0, T\left[{ }^{\circ} \mathrm{C}\right]\right)}{\max \left(0, T\left[{ }^{\circ} \mathrm{C}\right]\right)+\stackrel{\text { calc }}{h}_{T}},
$$

where the half-saturation constant is set to $h_{T}^{\text {calc }}=2{ }^{\circ} \mathrm{C}$. As coccolithophores are reported to have generally higher phosphorus affinity but lower nitrogen acquisition capacity with respect to other phytoplankton (Riegman et al., 2000; Paasche, 1998), limitation of these nutrients has an opposed impact on the rain ratio. This is reflected in our combined nutrient limitation factor for calcification, which is obtained from the phosphorus and nitrogen limitation of nanophytoplankton (Eqs. 241 and 240) as

$$
\stackrel{\text { calc }}{l_{\langle\mathbb{N P}\rangle}}=\min \left(1-{ }^{\text {nano nano }}, l_{\mathbb{P}}, l_{\mathbb{N}}\right) .
$$


Uptake limitation of suspension and deposit feeders by overcrowding is given by a nested Michaelis-Menten response to the respective biomass:

$$
\begin{aligned}
& \stackrel{\chi}{p}_{\text {crowd }}=\max \left(0, \stackrel{\chi}{Y}_{\mathbb{C}}-\stackrel{\chi}{p}_{\mathbb{C}}\right) \frac{\stackrel{\chi}{Y_{\mathbb{C}}-\stackrel{\chi}{p}_{\mathbb{C}}}}{{\underset{\chi}{Y}}_{\mathbb{C}}-\stackrel{\chi}{p}_{\mathbb{C}}+\stackrel{\chi}{h}_{\text {sat }}}, \\
& \stackrel{\chi}{l}_{\text {crowd }}=1-\frac{\stackrel{\chi}{p}_{\text {crowd }}}{{\stackrel{\chi}{p_{\text {crowd }}}+\stackrel{\chi}{h}_{\text {crowd }}}_{\text {che }}} .
\end{aligned}
$$

\subsection{Stoichiometric adjustments}

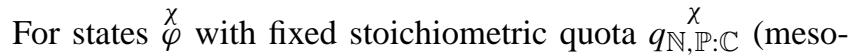
zooplankton, benthic bacteria and predators) the process rates are complemented by release fluxes that regulate imbalances in order to preserve the fixed reference quotas as follows:

$$
\begin{aligned}
& \left.\frac{\partial \stackrel{\chi}{\varphi_{\mathbb{C}}}}{\partial t}\right|_{\mathrm{rel}}=\max \left(\left.\widetilde{\frac{\partial}{\varphi_{\mathbb{C}}}}\right|_{\mathrm{bgc}}-\left.\frac{1}{q_{\mathbb{P}: \mathbb{C}}} \frac{\partial \hat{\varphi}_{\mathbb{P}}}{\partial t}\right|_{\mathrm{bgc}}\right.
\end{aligned}
$$

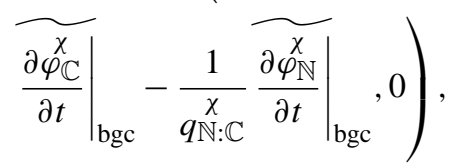

$$
\begin{aligned}
& \left.\frac{\partial \stackrel{\chi}{\varphi}_{\mathbb{N}, \mathbb{P}}}{\partial t}\right|_{\text {rel }}=\max \left(\widehat{\left.\frac{\partial \stackrel{\chi}{\varphi}_{\mathbb{N}, \mathbb{P}}}{\partial t}\right|_{\text {net }}-\left.q_{\mathbb{N}, \mathbb{P}: \mathbb{C}}^{\chi} \frac{\overline{\partial \varphi_{\mathbb{C}}}}{\partial t}\right|_{\text {bgc }}}\right),
\end{aligned}
$$

where $\left.\widetilde{\frac{\partial}{\varphi_{\mathbb{C}}}} \frac{}{\partial t}\right|_{\text {net }}$ are the comprehensive biogeochemical process rates prior to adjustments:

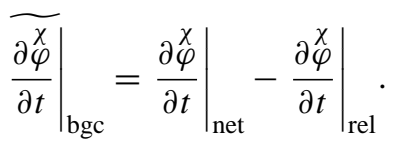

\section{Implementations}

Most ecosystem models are tightly bound to a specific physical, hydrodynamic driver that is usually three-dimensional and consequently computationally heavy and cumbersome to test and implement. The ERSEM model comes as an independent library and can in principle be coupled to any physical driver with comparatively little effort. In fact, coupled configurations exist for a variety of drivers in one- or three-dimensional settings, amongst which are the NEMO ocean engine (Madec, 2008), the POLCOMS model for shelf seas (Holt and James, 2001), and the GOTM/GETM model (Burchard et al., 2006). While for realistic implementations a full-scale three-dimensional configuration is required, for the stages of process development and qualitative analysis of the functioning of the modelled ecosystem, zero- or onedimensional frameworks are often beneficial as they provide a light-weight implementation that is easier to grasp, much faster to run, amenable to sensitivity analysis and quicker to analyse.

The model distribution itself includes drivers for two idealized systems: the first is a simple zero-dimensional implementation of mesocosm type called ERSEM-Aquarium with a pelagic box overlying a benthic box, each of them with internally homogeneous conditions. This is essentially a test environment for new users and fast process assessment requiring no external software for the ocean physics. The second is a driver for the vertical one-dimensional GOTM model (http://www.gotm.net - Burchard et al., 2006). It is a more realistic system allowing for full vertical structures in a comparatively lightweight software environment that is capable of running in serial mode on any standard desktop or laptop. It requires a copy of the GOTM code with minor modifications to accommodate ERSEM, which can be obtained for the stable release or the development release of GOTM (see Sect. 10). Here, we use the zero-dimensional framework to illustrate the carbon fluxes through the model food web under contrasting environmental conditions (Sect. 7.1) and the one-dimensional implementation to demonstrate the model capacity to reflect the lower trophic level of the marine ecosystem under varying conditions at three different sites, underpinned by a brief validation against in situ time-series data (Sect. 7.2).

Beyond these simpler test cases, the ERSEM model has been implemented in various full-scale three-dimensional applications from coastal to global scales cited above. The descriptions of these configurations would exceed the scope and volume of this paper and are given in the respective publications, but for completeness we give a short example of a simulation based on a previously published configuration in order to illustrate the full potential of the model (Sect. 7.3).

All simulations presented in this section were performed using the same parametrization, which is given in the Supplement. This parametrization was developed using size as the main trait to scale the metabolic rates of the pelagic functional groups more widely than in previous parametrizations (Baretta-Bekker et al., 1997; Blackford et al., 2004) and respects the conventional restriction of the food matrix suggested in Eq. (33). A table with all parameter values, their mathematical symbols as used in Sects. 2 to 6, and the corresponding name in the model code and namelists is given in the Supplement.

\subsection{ERSEM-Aquarium}

The simulation of mesocosm-type environments is supported through the ERSEM-Aquarium model. The model simulates two zero-dimensional boxes, a pelagic box, which is characterized by its mid-depth below the surface and by the geographical location, and a benthic box beneath it. Seasonal 

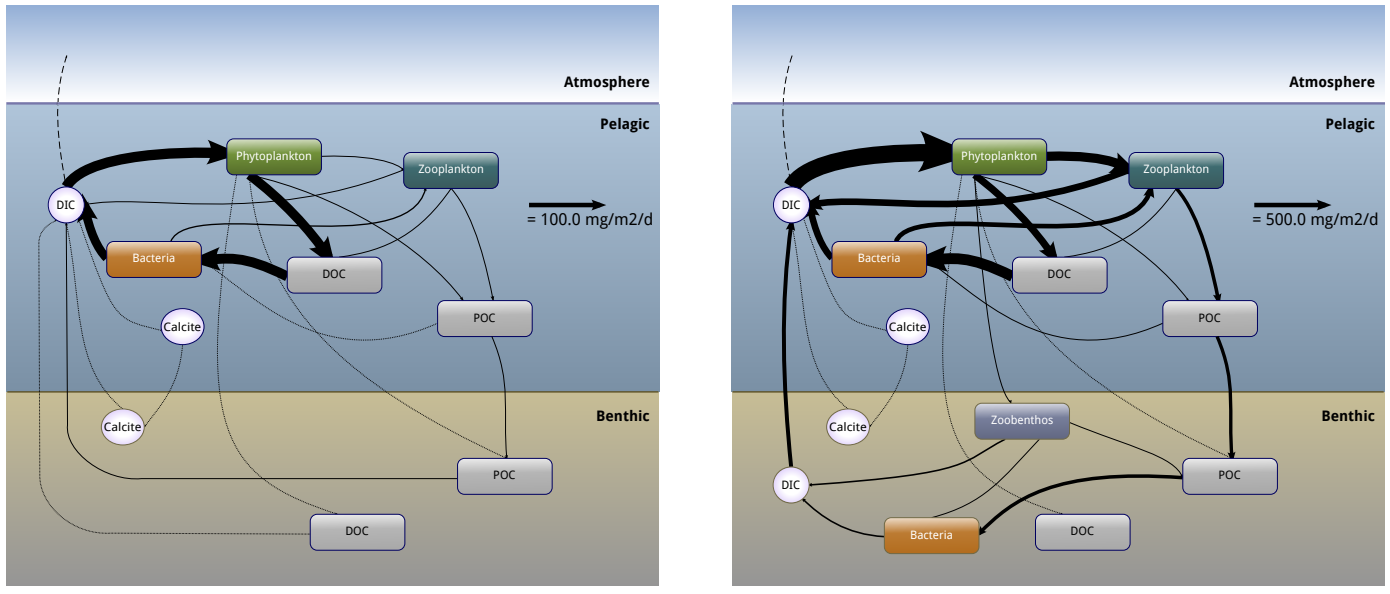

Figure 6. Carbon fluxes in ERSEM under oligotrophic (left panel) and eutrophic (right panel) conditions. The flux amount is proportional to arrow thickness. (Note the different scales of the arrow sizes.)

variations in temperature and salinity can be imposed as cosine functions between an extreme value at the first of January in the beginning of the simulation and a second extreme after half a year. The light field can be imposed in the same way as cosine oscillation between two prescribed extreme values, or extracted from the prescribed geographical position using a standard astronomical formula ignoring cloud cover. Additionally, diurnal oscillations of temperature and light can be superimposed in cosine form by prescribing a daily excursion between midday and midnight. It should be noted that this framework is not designed to deliver realistic simulations of the marine environment in a particular location, but rather to aid the development and quick evaluation of process studies or to study the model system behaviour in a simplified context without additional complicating factors.

Figure 6 illustrates the carbon fluxes between model compartments for two different simulations using ERSEMAquarium. The first is configured as a representation of tropical oligotrophic conditions characterized by deep and warm waters with high irradiance and low nutrients, while the second roughly corresponds to the shallow coastal eutrophic waters of the southern North Sea with strong nutrient supply and comparatively low light. Both configurations are run for 1000 years in order to achieve full equilibrium between the benthic and pelagic environments. The former uses the simple benthic closure scheme for remineralization (Sect. 5.1.5), which is more appropriate for deep water configurations where the impact of the benthos is of lesser importance, while the latter uses the full benthic model (Sect. 4). All configuration files necessary to replicate these runs are given in the Supplement. Figure 6 gives flux magnitudes in the modelled food web directly scaled from the annual average of the last year of each simulation. The experiment highlights the substantial quantitative production difference between the two systems. In addition, it clearly shows the qualitative shift in the model food web under the contrasting conditions. In the olig- otrophic case most of the gross production is excreted to dissolved matter due to strong nutrient limitation. This leads to a microbe-dominated scenario with bacteria as the main food source for the predators and only small amounts of carbon entering the second trophic level, leading to negative community production and low deposition of biomass to the sediments. In the eutrophic case production levels are increased by an order of magnitude. The assimilated carbon is used more efficiently by phytoplankton fuelling substantial secondary production with autotrophs as the main food source of zooplankton and significantly more biomass exported to the sediments, resulting in positive community production.

\subsection{GotmErsem - a model framework for the water column}

The GotmErsem framework provides the possibility to include a more realistic physical environment in the simulations with opposing gradients of nutrient supply from depth and shortwave radiation attenuated as it penetrates through the water column. The GOTM model is a one-dimensional water column model including a variety of turbulence closure schemes for vertical mixing (Burchard et al., 2006). Here, we show three implementations using this framework in contrasting environments to demonstrate the portability of the ERSEM model, one for the Oyster Grounds in the southern North Sea, a typical shelf sea site, one at the L4 site in the western English Channel representative of a mid-latitude site with mixed waters of both oceanic and coastal origin, and one in the oligotrophic sub-tropics at the Bermuda Atlantic Time-series Study site. Each of these sites is supported by extensive in situ data sets for model evaluation. Full configuration files to run these simulations are provided in the Supplement. The validation against in situ data was performed by sub-sampling the daily averaged model output for each in situ data sample. It is presented in target 
diagrams (Jolliff et al., 2009) for each site showing statistically robust metrics (e.g. Daszykowski et al., 2007) to account for the underlying non-Gaussian asymmetric data distributions and in order to avoid spurious overweighting of outliers. The metrics provided are the median bias (median $\left(M_{i}-D_{i}\right) ; M_{i}$ : model sample; $D_{i}$ : data sample) on the ordinate and the unbiased median absolute error (MAE', me$\left.\operatorname{dian}\left[\operatorname{abs}\left(M_{i}-D_{i}-\operatorname{median}\left(M_{i}-D_{i}\right)\right)\right]\right)$ on the abscissa. Both are normalized with the inter-quartile range (IQR) for the scale of the in situ data and the Spearman or rank correlation is represented by the colour code for each data set. The sign on the abscissa is given by the relation of IQRs $\left(\operatorname{sign}\left(\operatorname{IQR}\left(M_{i}\right)-\operatorname{IQR}\left(D_{i}\right)\right)\right)$.

All three sites are forced with data from the ERA-Interim reanalysis (Dee et al., 2011) at the atmospheric boundary condition. The L4 and Oyster Ground configurations use surface pressure data to introduce tidal mixing into the idealized one-dimensional set-ups. The BATS and L4 sites were additionally relaxed towards temperature and salinity profiles from CTD measurements (BATS - Steinberg et al., 2001, L4 - Harris, 2010) in order to compensate for the missing hydrodynamic impacts of lateral advection and diffusion. Initial conditions for the sites were derived from the concurrent in situ data where available. As for the ERSEM-Aquarium simulations, the benthic remineralization closure was used for the deep, oligotrophic BATS site, while for the shallow eutrophic sites L4 and Oyster Grounds, the full benthic model was used.

\subsubsection{Oyster Grounds $-\left(54^{\circ} 24^{\prime} 36^{\prime \prime} \mathrm{N}, 4^{\circ} 1^{\prime} 12^{\prime \prime} \mathrm{E}\right)$}

This site is located in the southern North Sea and is influenced by the English Channel and surrounding coastal waters, with seasonal stratification in most summers and an accentuated spring bloom at the onset of stratification that depletes the nutrients from the comparatively stable and isolated water surface layer (Baretta-Bekker et al., 2008).

A comparison with smart buoy data for the years 2000 2009 (Greenwood et al., 2010) reveals a good representation of the local seasonal cycle (Fig. 7). Simulations do not show significant bias in any of the variables, while the MAE' is significantly lower than the in situ data variability $(\approx 0.75$ of the IQR of the in situ data for chlorophyll $a, \approx 0.25$ silicate and phosphate, and virtually 0 for oxidized nitrogen). Correlations are high for the nutrients $(>0.6)$ but comparatively low for chlorophyll $a(>0.2)$. The lower skill for the latter is partly caused by a weaker secondary bloom in summer in the simulations compared to the observations and comparatively low observational coverage over the first years of the simulation, leading to potential overstressing of singular events in the data sampling and giving a spurious picture of the seasonal cycle when compared to the more consistently covered last 3 years of the period shown. In addition, some deficiencies in the model simulations are to be expected, as the Oyster Grounds site is characterized by strong lateral in- fluences including estuarine, coastal, and channel waters that include strong direct impacts on the nutrient concentrations in the area that can not be captured in this idealized setting. Particularly in the stratified season in summer, these lateral effects dominate the surface water signal, while the deeper part of the depression is essentially isolated from the surface layer (Weston et al., 2008).

\subsubsection{L4 - western English Channel $\left(50^{\circ} 15^{\prime} \mathrm{N}, 4^{\circ} 13^{\prime} \mathrm{W}\right)$}

The L4 site is a long-term monitoring station near the northern coast of the western English Channel. Similar to the Oyster Grounds site, it is seasonally stratified and generally nutrient depleted in summer, but highly affected by episodic events of freshwater inputs of riverine origin (Smyth et al., 2010).

Figure 8 shows the seasonal cycles of oxidized nitrogen, phosphate, and chlorophyll $a$ at the sea surface for the model simulations and for the in situ data (Smyth et al., 2010 http://www.westernchannelobservatory.org.uk/) for the years 2007-2011. The model follows the seasonal cycle of nutrient depletion in summer and nutrient resupply in winter revealed by the data in all three nutrients shown. Also, the results for chlorophyll $a$ follow the bulk seasonality represented by the in situ data, but show deficiencies in capturing the episodic peaks, which appear misplaced with respect to the measurements. Possible reasons for these shortcomings include the absence of physical and biogeochemical impacts of lateral processes in such an idealized one-dimensional setting as well as a sub-optimal representation of the local phytoplankton community by the parametrization adopted consistently across the contrasting environments. Nevertheless, the model skill expressed in the overall statistics is considerable. The bias and MAE' for all four variables fall well below the variability of the in situ data. Chlorophyll $a$ shows a relative bias of about 0.25 and a relative unbiased error of little less than 0.5 , while the three nutrients show an error and bias very close to 0 .

\subsubsection{BATS - Bermuda, Sargasso Sea $\left(31^{\circ} 40^{\prime} \mathrm{N}\right.$, $64^{\circ} 10^{\prime} \mathrm{W}$ )}

This site in the Sargasso Sea is characterized by a weak geostrophic flow with net downwelling. Strong stratification separates the nutrient-poor surface waters from the nutrientrich deep water, with the exception of the passing of cold fronts in winter which cause substantial convective mixing with accompanying nutrient entrainment (Steinberg et al., 2001). This is illustrated in the left panel of Fig. 9, which shows the seasonal cycle of chlorophyll $a$ from model simulations (on top) and in situ data. The mixing events triggering autotrophic growth initially spread over the upper part of the water column, but they are limited to a rather marked deepchlorophyll $a$ maximum at around $100 \mathrm{~m}$ depth when stratification sets in. Interannual variability at the site is dominated 

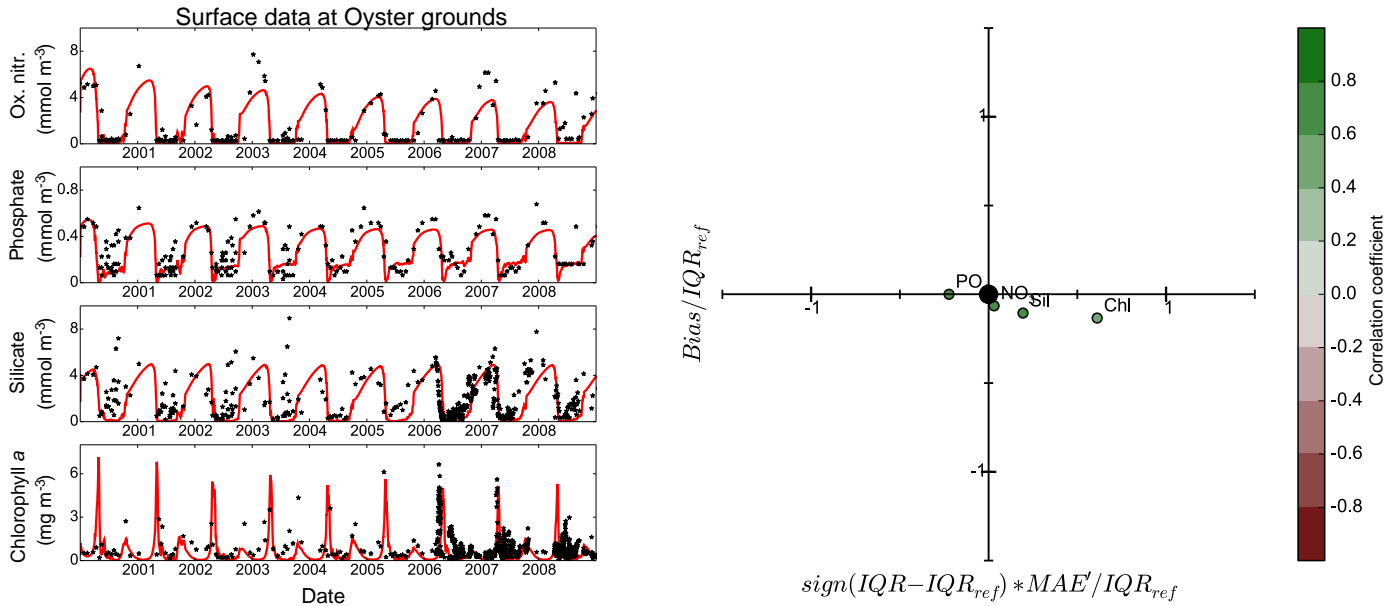

Figure 7. Simulation results vs. in situ data at the Oyster Grounds - left panels: model time series (red lines) vs. in situ measurements (black dots) for oxidized nitrogen, phosphate, silicate, and chlorophyll $a$ (top to bottom panels); right panel: target diagram with bias (abscissa), MAE' (ordinate), and Spearman correlation (colour code) for oxidized nitrogen $\left(\mathrm{NO}_{3}\right)$, phosphate $\left(\mathrm{PO}_{4}\right)$, silicate $($ Sil), and chlorophyll $a$ (Chl). The observations consist of ship-based data collected by Rijkswaterstaat as part of Dutch national monitoring programme MWTL (see publicwiki.deltares.nl/display/OET/Dataset+documentation+MWTL) and SmartBuoy data collected by Cefas in collaboration with Rijkswaterstaat (Greenwood et al., 2010; http://www.cefas.co.uk/publications-data/smartbuoys).
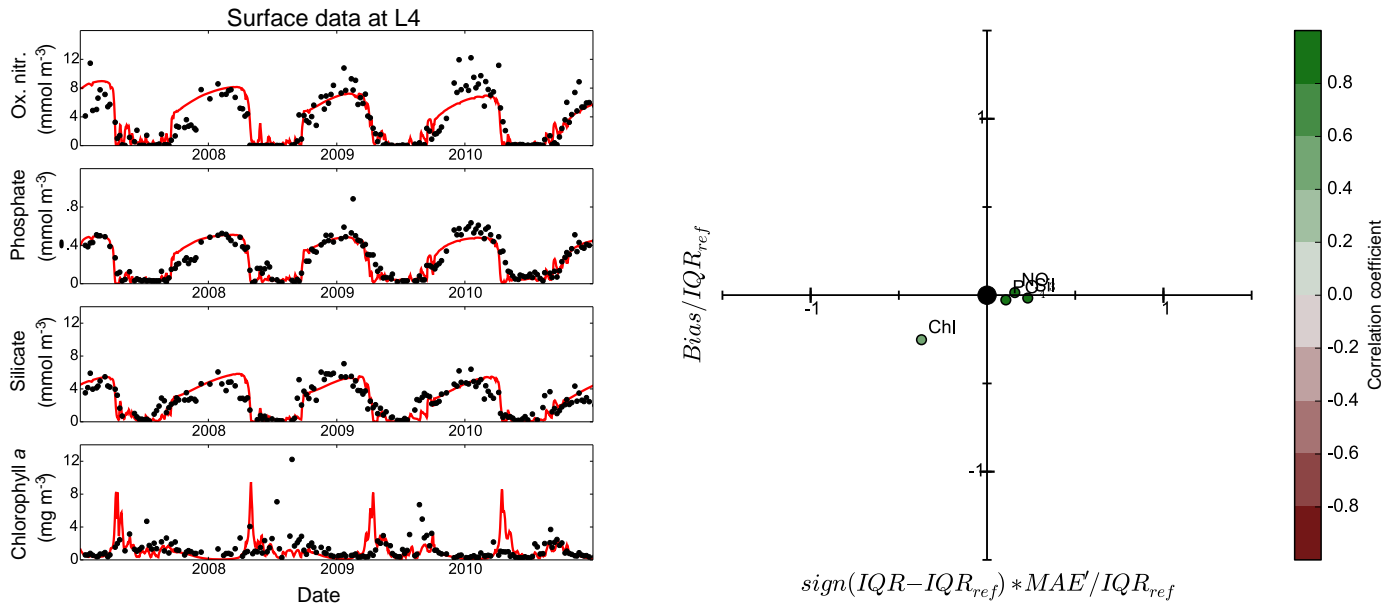

Figure 8. Simulation results vs. in situ data at the L4 site - left panels: model time series (red lines) vs. in situ measurements (black dots) for oxidized nitrogen, phosphate, silicate, and chlorophyll $a$ (top to bottom panels); right panel: target diagram with bias (abscissa), MAE' (ordinate), and Spearman correlation (colour code) for oxidized nitrogen $\left(\mathrm{NO}_{3}\right)$, phosphate $\left(\mathrm{PO}_{4}\right)$, silicate (Sil), and chlorophyll $a(\mathrm{Chl})$.

by the varying strength of the sub-tropical storm events in spring that cause strong vertical mixing which can reach up to $200 \mathrm{~m}$ depth, resulting in variable levels of nutrient entrainment, largely captured by the model. A summary of the validation against the extensive in situ data available at BATS (Bermuda Time Series Study - Steinberg et al., 2001) for the years 1990-2008 is given in the target diagram on the right of Fig. 9. In contrast to the two shallow sites, in situ data in this case are vertically resolved, which was respected in the matching procedure.

Bias and MAE' for all variables do not exceed the variability of the in situ data. Both metrics are very close to zero for the nitrate, phosphate, and chlorophyll $a$, and in general most metrics stay below $50 \%$ of the in situ variability, with the exception of the bias for oxygen and the MAE' for phosphate. The latter is caused by an underestimated aeration of the water column and a weaker vertical gradient in phosphate for the model (not shown). However, some weaknesses in the simulation of the vertical distributions are to be expected given the absence of explicit lateral dynamics and the resulting vertical flows. Correlations lie between 0.4 and 0.6, reflecting the overall satisfactory model performance. 

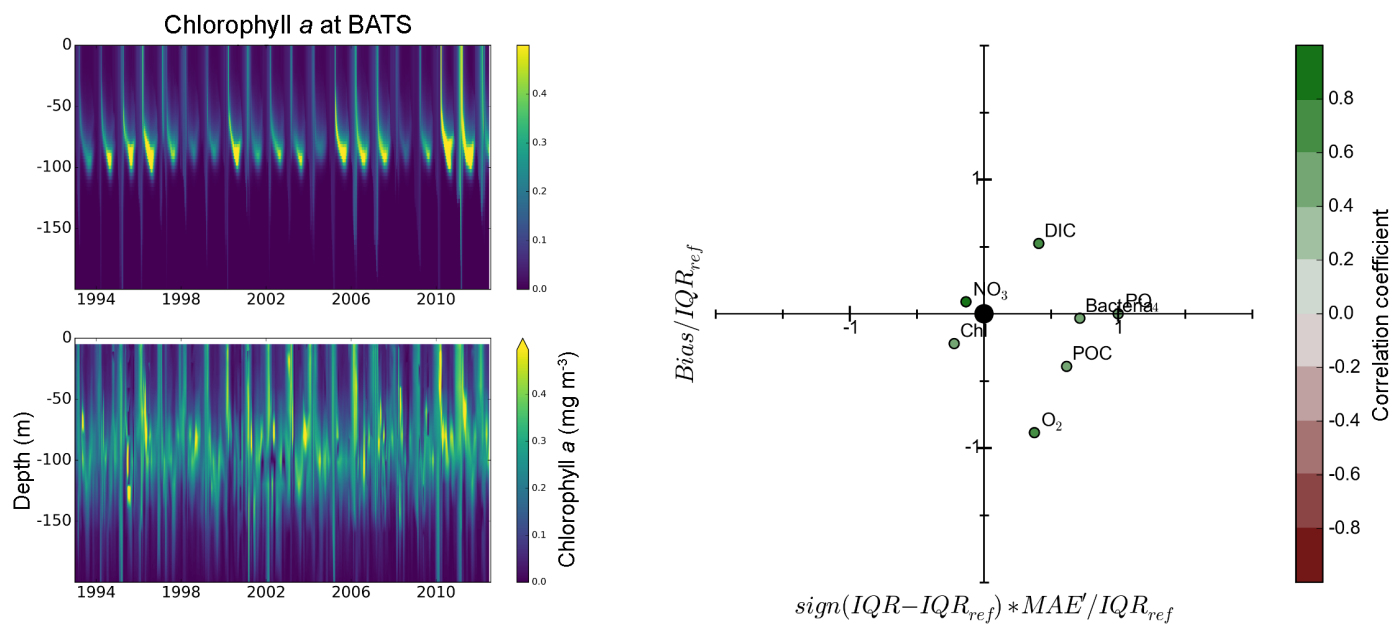

Figure 9. Simulation results vs. in situ data at BATS - left panels: chlorophyll $a$ concentrations (top panel - model, bottom panel - interpolated HPLC data); right panel: target diagram with bias (abscissa), MAE' (ordinate), and Spearman correlation (colour code) for oxidized nitrogen $\left(\mathrm{NO}_{3}\right)$, phosphate $\left(\mathrm{PO}_{4}\right)$, silicate (Sil), dissolved inorganic carbon (DIC), dissolved oxygen $\left(\mathrm{O}_{2}\right)$, chlorophyll $a(\mathrm{Chl})$, and particulate organic carbon (POC).
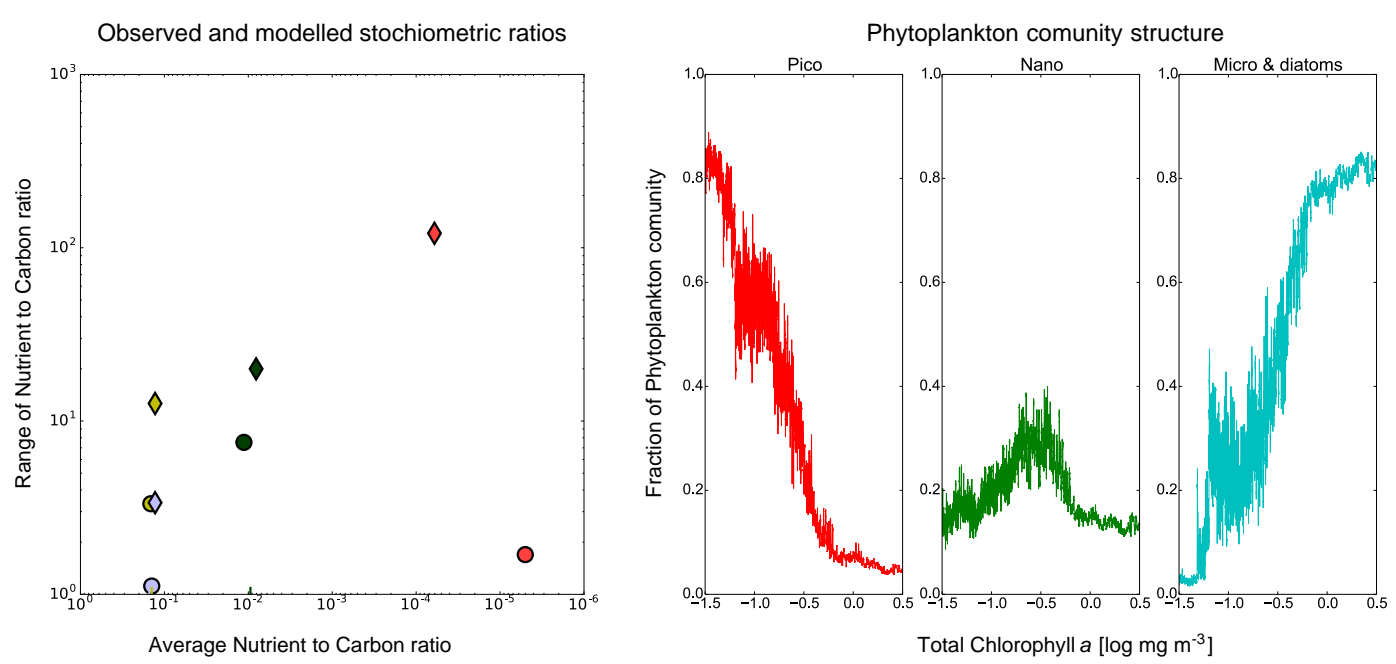

Figure 10. Emergent properties of the simulations across the three one-dimensional sites. Left panel: range (ordinate) and mean (abscissa) of internal stoichiometric ratios of phytoplankton - nitrogen (yellow), silicate (blue), phosphorus (green), and iron (red). Data (diamonds, Moore et al., 2013), assembled one-dimensional model simulations (circles); right panels: community fraction of total chlorophyll $a$ from assembled one-dimensional model simulations. Picophytoplankton (red), nanophytoplankton (green), and microphytoplankton and diatoms (cyan).

\subsubsection{Properties emerging from simulations at all three sites}

In order to give an impression of the functioning of the ecosystem dynamics across the three sites, Fig. 10 shows a comparison between some ecosystem properties emerging from data meta-analysis and model simulations, namely the internal stoichiometric quotas of nutrients with respect to carbon in phytoplankton and the phytoplankton community structure. On the left of Fig. 10 we show the range of the internal stoichiometric quotas of nitrogen, phosphorus, silicate, and iron with respect to carbon on the abscissa plotted against the average quotas for phytoplankton on the ordinate as an indicator of the modelled phytoplankton plasticity in response to nutrient limitation. Quotas from the simulations (circles) are compared to the results of a meta-analysis (diamonds) provided by Moore et al. (2013) based on observed internal stoichiometric phytoplankton quotas from the scientific literature. Results for the three macronutrients are consistent in that the average quotas are well matched, while the stoichiometric range is underestimated by approximately half an order of magnitude. This is to be expected given that the 
case studies included in the model simulations do not cover the full range of natural variability of marine environments. Results for iron show substantial differences in range and average state. The mismatch in average state can be attributed to the fact that the present parametrization of the iron cycle took into consideration the works of Timmermans et al. (2005) and Veldhuis et al. (2005), which reported comparatively low iron to carbon quotas, but were not considered in the above meta-analysis, while the huge discrepancy in range is caused by the absence of substantial iron limitation in the sites of the case studies.

The right-hand side panel of Fig. 10 shows the sizefractionated contribution of each phytoplankton group to total chlorophyll $a$ across the three sites as a running average over the ordered model samples from all three sites collectively. The procedure is analogous to the meta-analysis provided by Hirata et al. (2011). The results show a domination of the phytoplankton community by picophytoplankton at low chlorophyll $a$ and by large phytoplankton at high chlorophyll $a$. Nanophytoplankton is present throughout the chlorophyll $a$ range, reaching a maximum at intermediate values. The emerging modelled community structure compares well to the meta-analysis (compare Fig. $2 \mathrm{a}-\mathrm{c}$ therein), particularly considering the limited range of marine environments considered in this exercise.

\subsection{A full-scale implementation for the north-western European shelf}

The previous case studies demonstrate the capability of the model to represent the marine ecosystem, with a focus on small-scale ecosystem processes. Nevertheless, the full potential of the model unfolds in full-scale applications of coupled dynamical systems linked to hydrodynamic models capturing the full advection and diffusion of the biogeochemical states and thus providing a complete synoptic picture of the large-scale biogeochemical cycles and the marine environment. A full description of these systems would exceed the scope of this particular paper. Nevertheless, we give here a brief overview of the model performance on a simulation of the north-western European shelf seas using the POLCOMS model for shelf sea circulation (Holt and James, 2001), based on a hindcast configuration identical to the one used and described in Holt et al. (2012) and Artioli et al. (2012) but using the most recent model version presented in this work and the same parametrization as in the above examples.

The left-hand side panel of Fig. 11 shows the mean opticaldepth-averaged chlorophyll $a$ field of the area to illustrate the model domain as used in the validation exercise, and also to give an idea of the ecosystem characteristics of the area. Model simulations were validated against in situ data for oxidized nitrogen, phosphorus, chlorophyll $a$, oxygen, and salinity retrieved from the ICES database (ICES, 2009) for the period of 1970-2004 using the same metrics as above, summarized in a target diagram on the right of Fig. 11. Re- sults are consistent with the validation results of the onedimensional sites with both bias and MAE' generally less than $50 \%$ of the in situ variability, and correlations $>0.4$ for all variables, confirming the good performance of the model dynamics in a realistic large-scale simulation.

\section{Development and testing framework}

In addition to the zero- and one-dimensional ERSEM implementations, a framework is provided with the model that allows developers and users of the code to analyse and plot the result of calls to individual ERSEM procedures from Python. This facility is supported through Fortran-C interoperability, which arrived with the Fortran 2003 standard (ISO/IEC 1539-1:2004(E)) and http://docs.python.org/ 2/library/ctypes.html. ERSEM test harnesses consist of the ERSEM library and a set of $\mathrm{C}$ wrappers, which are jointly compiled as a shared library. A Python interface to the shared library permits access to Fortran data structures and procedures from Python. This allows developers and users of the code to quickly interrogate the validity and behaviour of individual procedures, without first reimplementing them in a second language, and without running the full model. Here we illustrate this feature by examining the photosynthesis model implemented in ERSEM.

The photosynthesis model used in ERSEM is based on Geider et al. (1997), and is described in Sect. 3.1. In the model, photosynthetic cells are able to regulate their chlorophyll $a$ to carbon ratio in response to changes in irradiance, temperature, and silicate (in the case of diatoms) by modifying the proportion of photosynthate that is directed towards chlorophyll biosynthesis $(\stackrel{\chi}{\rho}$; see Eq. 10$)$. Balanced growth is achieved when cells are fully acclimated, in which case

$$
\frac{\mathrm{d}}{\mathrm{d} t}\left(\begin{array}{l}
\stackrel{\chi}{P}_{\mathcal{C}} \\
\frac{\chi}{P_{\mathbb{C}}}
\end{array}\right)=0 .
$$

Chlorophyll $a$ biosynthesis is assumed to be up-regulated in response to a reduction in irradiance and down-regulated in response to an increase in irradiance. Through this process, cells are able to balance the rate of energy supply through light absorption and energy demands for growth. The maximum, light-saturated photosynthesis rate $\stackrel{\chi}{g}(T)$ is assumed to be independent of changes in irradiance, which is consistent with observations which indicate that Rubisco content is relatively invariant with respect to changes in irradiance (Sukenik et al., 1987), and the hypothesis that these cells are adapted to survive and reproduce in dynamic light environments (Talmy et al., 2014).

Using the ERSEM testing framework, it is possible to investigate this process in isolation. Model cells can be artificially acclimated to a given set of environmental conditions by finding a value for $\underset{\mathcal{C}: \mathbb{C}}{\chi}$ which satisfies Eq. (271). 

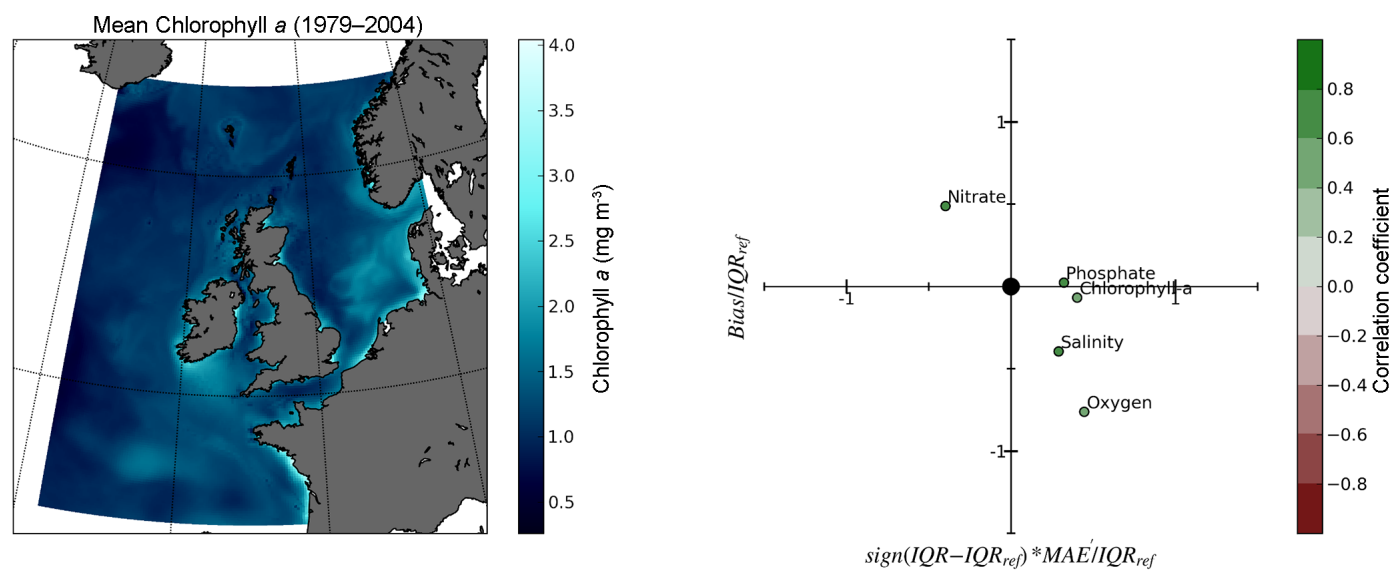

Figure 11. The ERSEM model in a simulation for the north-western European shelf seas - left panel: optical-depth-averaged chlorophyll $a$; right panel: hindcast simulation vs. in situ data.

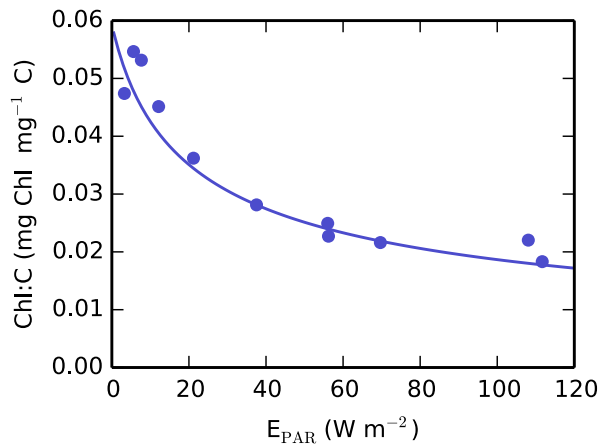

Figure 12. Chlorophyll $a$ to carbon ratio of diatoms as a function of PAR under the condition of balanced growth (Eq. 271). The solid line represents output from the model. Black circles show data for nutrient-replete cultures of Thalassiosira pseudonana, digitally extracted from Geider et al. (1997) using Plot Digitizer Version 2.6.6 (see http://plotdigitizer.sourceforge.net).

Figure 12 shows a plot of $q \stackrel{\chi}{\mathcal{C}: \mathbb{C}}$ vs. I IPAR for fully photoacclimated diatoms in ERSEM. Cells were acclimated to a given irradiance by holding cellular carbon fixed and varying the cellular chlorophyll $a$ content within the range $\stackrel{\chi}{q}_{\text {min }_{\mathcal{C}: \mathbb{C}}} \leq$ $\stackrel{\chi}{q} \leq \stackrel{\chi}{q}_{\varphi \max }$ in order to achieve balanced growth. Using the testing framework, the model can be compared with observations in order to sanity check the validity of the implementation, or parametrized against observations using curve fitting procedures. In Fig. 12, observations for the diatom T. Pseudonana have been overlaid. No attempt was made to fit the curve to this particular set of observations, although the fit appears reasonable. The parameter set is the same as used in the simulations of Sect. 7 and is given in the Supplement.

Diatoms are a physiologically and morphologically diverse group, which are characterized by their requirement for silicate, which they use to construct their cell wall. It is perhaps unsurprising that model fits to photosynthesisirradiance curves for different diatom species result in a range of parameter values, including differences in the maximum light-saturated carbon-specific photosynthesis rate as a function of temperature and the initial slope of the photosynthesis-irradiance curve (e.g. Geider et al., 1997). Ultimately, many of these differences arise due to differences in organism morphology and physiology, with, for example, different pigment complements or levels of investment in biosynthesis being reflected in derived parameter values. These within-group variations pose a perennial problem to the development of marine ecosystem and biogeochemical models. The diatom group in ERSEM is designed to be representative of diatoms as a whole and to reflect the important biogeochemical role these organisms perform in nature.

ERSEM includes four phytoplankton functional groups: diatoms, which are characterized by their requirement for silicate, and three further groups which are characterized according to their size. These are the pico-, nano-, and microphytoplankton. The choice to characterize groups according to their size reflects the importance of size as a physiological trait (Litchman et al., 2007, 2010), which influences an organism's competitive ability through its effect on nutrient acquisition, carbon and nutrient storage, the intracellular transport of solutes, photosynthesis rates through pigment packaging effects, and susceptibility to predation (e.g. Chisholm, 1992; Finkel et al., 2010).

Using ERSEM's testing framework, it is possible to demonstrate how this classification impacts the competitive ability of the four photosynthetic groups represented in the model. Figure 13 shows photosynthesis-irradiance curves for ERSEM's four phytoplankton groups under the condition of balanced growth. As with the diatoms, the use of a single parameter set for each size-based group ignores within-group variations that are observed in nature. It is important to take 


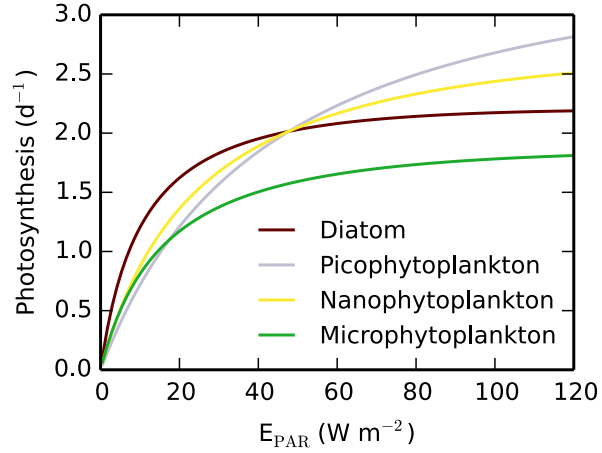

Figure 13. Phytoplankton growth over PAR for the four phytoplankton types.

such abstractions into consideration when interpreting model outputs.

This example illustrates how ERSEM's testing framework can be used to study and check the implementation of different processes within the code. Importantly, this is achieved without having to rewrite sections of the code in a second language with visualization capabilities, which is an inherently error-prone procedure. This capability is designed to complement the zero-dimensional and one-dimensional drivers that simulate more complex time-varying environments in which it is often difficult to study processes in isolation.

\section{Optional choices}

In the following section we provide an overview of the main optional choices in the model configuration. Options that involve major structural changes which alter the number of state variables or add substantial functionality are activated by preprocessor definitions that need to be included at compile time. These include

- the model of bacterial decomposition;

- the inclusion of the iron cycle;

- the light attenuation model; and

- the calcification model.

Other options can be triggered at run time via namelist parameters in the files include/ersem_pelagic_switches.nml and include/ersem_benthic_switches.nml without the need for a recompilation of the model code. These include the choice of the alkalinity description of the model and the choice of the benthic model.

\subsection{The iron cycle}

The use of the iron cycle in the model including growth limitation of phytoplankton by iron is activated by the IRON pre- processor key. It involves additional state variables for dissolved inorganic iron and iron components of the four phytoplankton types, two particulate matter types in the pelagic and one particulate matter type in the benthos.

\subsection{Calcification}

The use of the calcification sub-module (Sect. 3.6) is activated by the CALC preprocessor key. Its computational impact is limited, adding a single pelagic and single benthic state to the list of state variables.

\subsection{The model of bacterial decomposition}

Two options are included for the modelling of the decomposition of organic matter (see Sect. 3.3). By default, the bacteria sub-model presented in Allen et al. (2002) with a basic microbial food web and implicit decomposition is used. Enabling the DOCDYN preprocessing key, the model for dynamic decomposition of organic matter is activated which uses fully explicit recycling of organic matter and includes the recalcitrant fraction of the DOC pool at the cost of an additional state variable.

\subsection{The light attenuation model}

Two options for light attenuation are available. The default choice is the legacy model based on apparent optical properties in the form of specific attenuation coefficients, while the recently developed model using inherent optical properties in the form of specific adsorption and backscatter coefficients and zenith angle needs to be activated by the IOPMODEL preprocessor key (see Sect. 3.9). The computational effort of the two models is comparable, but the latter involves the computation of the zenith angle and therefore requires the geographical coordinates and the current simulation date and time from the physical driver.

\subsection{Alkalinity}

The description of alkalinity in the model is given by the combination of two switches. The prognostic mode using an ocean tracer modified by biogeochemical processes affecting alkalinity is activated by setting ISWbioalk in include/ersem_pelagic_switches.nml to 1 . The diagnostic mode deriving alkalinity from salinity (and optionally temperature) is enabled by activating an adequate alkalinity regression by setting ISWtalk to a value between 1 and 4. (The different regression options are specified in the Supplement.) The recommended use for these modes is a combination of both modes or the purely progostic option with ISWbioalk $=1$ and ISWtalk $=5$ (see Sect. 3.8).

\subsection{The benthic model}

The full benthic model (Sect. 4) is activated by setting the ibenXin parameter in 
include/ersem_benthic_switches.nml to 2, while for $i b e n X i n=1$ (see Sect. 5.1.5) the benthic closure scheme is used. While the latter involves considerably fewer state variables and computations, the computational impact of this choice is largely negligible in one-dimensional and three-dimensional simulations, as the computational cost is dominated by the advection and diffusion of the pelagic states.

\section{Technical specifications and code availability}

The ERSEM 15.06 model is written in FORTRAN using the 2008 standard. Output is entirely based on netCDF and the output parsing scripts generating I/O FORTRAN code from plain text lists of variables are written in Python.

The model is distributed under the open-source GNU Lesser General Public License through a gitlab server and freely available upon registration through the http:// www.shelfseasmodelling.org web portal. There are no restrictions or conditions for the registration of individual users; the registration is merely implemented in order to keep track of the user base. The code repository is fully version controlled (using git) and features a bug tracking system open to users. The release code of this publication is available in the master branch of the repository as tag https://gitlab.ecosystem-modelling.pml.ac.uk/ ssb-gotm-ersem/ssb-ersem/tree/ERSEM-15.06. The GOTM version used in the simulations of this work is also tagged as "ERSEM-15.06" on the ERSEM enabled fork of the development version of GOTM, which can be downloaded from the same repository server. A quick start guide and user's reference manual are also provided along with the code.

The versioning convention used with this software refers to the year and month of the release.

\section{Conclusions}

In this paper we have provided a full mathematical description of an updated version of ERSEM, one of the most established marine ecosystem models currently in use in the scientific community and in operational systems. Case studies ranging from a mesocosm-type zero-dimensional experiment through three one-dimensional water column implementations to a brief three-dimensional full-scale example have illustrated the model dynamics in varying environments.

Qualitative and quantitative validation with in situ data for the basic ecosystem state variables chlorophyll $a$ and the macronutrients has demonstrated the capability of the model to represent ecosystems ranging from oligotrophic open oceans to eutrophic coastal conditions. An integral validation of each single component would exceed the scope of this paper, the main purpose of which is the detailed description of the model ingredients as a reference for scientists, developers and users. Nevertheless, examples of component validations have been published previously and are available in the literature (Artioli et al., 2012; Allen and Somerfield, 2009; Allen et al., 2007; de Mora et al., 2013). In addition the testing framework supplied within the model distribution allows for targeted analysis and validation of individual parts of the model down to the level of single equations directly without rewriting or extracting the model code. We have demonstrated this capability here on the example of the PI curve for phytoplankton growth.

The ERSEM 15.06 model is to our knowledge the only model currently available that provides the structure for simulating in one coherent system the biogeochemical cycles of carbon, the major macronutrients and iron (using variable stochiometric relationships), the carbonate system and calcification, the microbial food web and the benthic biogeochemistry.

While the range of processes included in the model brings the advantage of suitability for a whole range of applications as different as process studies, regional or global budgets of different chemical elements, habitat maps, or risk assessment of environmental hazard, it also points to one of the major drawbacks of the model, i.e. a comparatively heavy structure and high number of parameters that render it difficult to access for new users and hard to calibrate and parametrize. These problems are being addressed in a fully modular version of the model with streamlined process descriptions that is currently under development. It will allow for an arbitrary number of functional groups and easy replacement of individual sub-models, which can be tuned to the specific application at run time. These developments will be made available with the next release of the model.

\section{The Supplement related to this article is available online at doi:10.5194/gmd-9-1293-2016-supplement.}

Acknowledgements. The contributions of M. Butenschön, J. Clark, J. N. Aldridge, J. I. Allen, Y. Artioli, J. Blackford, G. Lessin, S. van Leeuwen, J. van der Molen, L. Polimene, S. Sailley, and N. Stephens were partly funded by the UK Shelf Seas Biogeochemistry programme (contract no. NE/K001876/1) of the National Environmental Research Council (NERC) and the Department for Environment Food and Rural Affairs (DEFRA).

M. Butenschön, J. Clark, J. I. Allen, Y. Artioli, J. Bruggeman, P. Cazenave, S. Ciavatta, S. Kay, G. Lessin, L. de Mora, S. Sailley, N. Stephens, and R. Torres were supported by the NERC National Capability in Modelling programme at the Plymouth Marine Laboratory. J. N. Aldridge, S. van Leeuwen and J. van der Molen were also supported through matched funding by DEFRA under contract C6164. J. Blackford and Y. Artioli were partly funded by the Regional Ocean Modelling project (contract no. NE/H017372/1) of the NERC UK Ocean Acidification research programme (UKOA). L. de Mora, M. Butenschön and J. I. Allen were partly funded by the Integrated Global Biogeochemical Modelling Network to support UK Earth System Research (i-MarNet, contract no. NE/K001345/1) project. M. Butenschön and L. Polimene were partly funded by the 
EU-FP7 MyOcean project (Grant Agreement 283367). J. I. Allen and S. Ciavatta were additionally supported by the UK - National Centre of Earth Observations (NCEO) of NERC.

We gratefully acknowledge the work of the development team of the original ERSEM versions I and II under the lead of J. W. Baretta providing the baseline versions this model has emerged from over the last 2 decades.

The SmartBuoy observations at the Oyster Grounds were collected as part of the Marine Ecosystem Connections (MECS) programme led by Cefas and funded by the Department for Environment, Food and Rural affairs (Defra) through contract E3205; see Biogeochemistry 113, Issue 1, 2013 for key publications from MECS. In situ observations at the L4 site were obtained from the Western English Observatory http://www.westernchannelobservatory.org.uk/). The data of the Bermuda Time Series Site were collected by the Bermuda Institute of Ocean Science Bermuda Time Series Study. Validation data for the north-eastern Atlantic were retrieved from the Dataset on Ocean Hydrography distributed by the International Council for the Exploration of the Sea (ICES).

Edited by: A. Ridgwell

\section{References}

Aksnes, D. L. and Egge, J. K.: A theoretical model for nutrient uptake in phytoplankton, Mar. Ecol.-Prog. Ser., 70, 65-72, doi:10.3354/meps070065, 1991.

Allen, J. I. and Somerfield, P. J.: A multivariate approach to model skill assessment, J. Marine Syst., 76, 83-94, doi:10.1016/j.jmarsys.2008.05.009, 2009.

Allen, J. I., Somerfield, P. J., and Siddonr, J.: Primary and bacterial production in the Mediterranean Sea: a modelling study, J. Marine Syst., 33-34, 473-495, doi:10.1016/S0924-7963(02)000726, 2002.

Allen, J. I., Blackford, J. C., Holt, J., Proctor, R., Ashworth, M., and Siddorn, J.: A highly spatially resolved ecosystem model for the North West European Continental Shelf, Sarsia, 86, 423-440, 2001.

Allen, J. I., Somerfield, P. J., and Gilbert, F. J.: Quantifying uncertainty in high-resolution coupled hydrodynamic-ecosystem models, in: Contributions from Advances in Marine Ecosystem Modelling Research, 27-29 June 2005, Plymouth, UK AMEMR, 64, 3-14, available at: http://www.sciencedirect.com/science/article/ pii/S0924796306001035 (last access: 14 August 2015), 2007.

Anderson, T. R., Hessen, D. O., Mitra, A., Mayor, D. J., and Yool, A.: Sensitivity of secondary production and export flux to choice of trophic transfer formulation, J. Marine Syst., 125, 4153, doi:10.1016/j.jmarsys.2012.09.008, 2013.

Artioli, Y., Blackford, J. C., Butenschön, M., Holt, J. T., Wakelin, S. L., Thomas, H., Borges, A. V., and Allen, J. I.: The carbonate system in the North Sea: sensitivity and model validation, J. Marine Syst., 102-104, 1-13, doi:10.1016/j.jmarsys.2012.04.006, 2012.

Artioli, Y., Blackford, J. C., Nondal, G., Bellerby, R. G. J., Wakelin, S. L., Holt, J. T., Butenschön, M., and Allen, J. I.: Heterogeneity of impacts of high $\mathrm{CO}_{2}$ on the North Western Eu- ropean Shelf, Biogeosciences, 11, 601-612, doi:10.5194/bg-11601-2014, 2014a.

Artioli, Y., Blackford, J. C., Nondal, G., Bellerby, R. G. J., Wakelin, S. L., Holt, J. T., Butenschön, M., and Allen, J. I.: Heterogeneity of impacts of high $\mathrm{CO}_{2}$ on the North Western European Shelf, Biogeosciences, 11, 601-612, doi:10.5194/bg-11601-2014, 2014b.

Aumont, O., Maier-Reimer, E., Blain, S., and Monfray, P.: An ecosystem model of the global ocean including $\mathrm{Fe}$, Si, P colimitations, Global Biogeochem. Cy., 17, 1-23, doi:10.1029/2001GB001745, 2003.

Barange, M., Merino, G., Blanchard, J. L., Scholtens, J., Harle, J., Allison, E. H., Allen, J. I., Holt, J., and Jennings, S.: Impacts of climate change on marine ecosystem production in societies dependent on fisheries, Nat. Clim. Change, 4, 211-216, doi:10.1038/nclimate2119, 2014.

Baretta, J., Ruardij, P., Billings, W. D., Golley, F., Lange, O. L., Olson, J. S., and Remmert, H. (Eds.): Tidal Flat Estuaries, vol. 71 of Ecological Studies, Springer Berlin Heidelberg, Berlin, Heidelberg, Germany, available at: http://link.springer.com/10.1007/ 978-3-642-73753-4 (last access: 14 August 2015), 1988.

Baretta, J. W.: Preface, J. Sea Res., 38, 169-171, doi:10.1016/S1385-1101(97)00054-3, 1997.

Baretta, J. W., Ebenhöh, W., and Ruardij, P.: The European regional seas ecosystem model, a complex marine ecosystem model, Neth. J. Sea Res., 33, 233-246, doi:10.1016/00777579(95)90047-0, 1995.

Baretta-Bekker, H., Bot, P., Prins, T., and Zevenboom, W.: Report on the second application of the OSPAR Comprehensive Procedure to the Dutch marine waters, Tech. rep., OSPAR Comission, London, 2008.

Baretta-Bekker, J. G.: Note of the editor, Neth. J. Sea Res., 33, 230 231, doi:10.1016/0077-7579(95)90046-2, 1995.

Baretta-Bekker, J. G., Baretta, J. W., and Rasmussen, E. K.: The microbial food web in the European Regional Seas Ecosystem Model, Neth. J. Sea Res., 33, 363-379, 1995.

Baretta-Bekker, J. G., Baretta, J. W., and Ebenhöh, W.: Microbial dynamics in the marine ecosystem model ERSEM II with decoupled carbon assimilation and nutrient uptake, J. Sea Res., 38, 195-211, doi:10.1016/S1385-1101(97)00052-X, 1997.

Bellerby, R. G. J., Olsen, A., Furevik, T., and Anderson, L. G.: Response of the surface ocean $\mathrm{CO}_{2}$ system in the Nordic Seas and northern North Atlantic to climate change, in: The Nordic Seas: An Integrated Perspective, edited by: Drange, H., Dokken, T., Furevik, T., Gerdes, R., and Berger, W., American Geophysical Union, Washington, D.C., USA, 189-197, doi:10.1029/158GM13, 2005.

Blackford, J., Artioli, Y., Kelly-Gerreyn, B., Martin, A., Tyrrell, T., and Somavilla, R.: Sub-model acidification-sensitive calcification rate, including user guide, Project Report, D2.2, EC FP7 MEECE - 212085, Plymouth Marine Laboratory, Plymouth, UK, 2010.

Blackford, J. C.: An analysis of benthic biological dynamics in a North Sea ecosystem model, J. Sea Res., 38, 213-230, doi:10.1016/S1385-1101(97)00044-0, 1997.

Blackford, J. C. and Burkill, P. H.: Planktonic community structure and carbon cycling in the Arabian Sea as a result of monsoonal forcing: the application of a generic model, J. Marine Syst., 36, 239-267, doi:10.1016/S0924-7963(02)00182-3, 2002. 
Blackford, J. C. and Gilbert, F. J.: $\mathrm{pH}$ variability and $\mathrm{CO}_{2}$ induced acidification in the North Sea, Symposium on Advances in Marine Ecosystem Modelling Research, Plymouth, England, 27-29 June 2005, J. Marine Syst., 64, 229-241, doi:10.1016/j.jmarsys.2006.03.016, 2007.

Blackford, J. C., Allen, J. I., and Gilbert, F. J.: Ecosystem dynamics at six contrasting sites: a generic modelling study, J. Marine Syst., 52, 191-215, 2004.

Bopp, L., Resplandy, L., Orr, J. C., Doney, S. C., Dunne, J. P., Gehlen, M., Halloran, P., Heinze, C., Ilyina, T., Séférian, R., Tjiputra, J., and Vichi, M.: Multiple stressors of ocean ecosystems in the 21st century: projections with CMIP5 models, Biogeosciences, 10, 6225-6245, doi:10.5194/bg-10-6225-2013, 2013.

Borges, A. V. and Frankignoulle, M.: Daily and seasonal variations of the partial pressure of $\mathrm{CO}_{2}$ in surface seawater along Belgian and southern Dutch coastal areas, J. Marine Syst., 19, 251-266, doi:10.1016/S0924-7963(98)00093-1, 1999.

Broecker, W. S. and Peng, T. H.: Tracers in the sea, Eldigio Press, New York, USA, 1982.

Broekhuizen, N., Heath, M. R., Hay, S. J., and Gurney, W. S. C.: Modelling the dynamics of the North Sea's Mesozooplankton, Neth. J. Sea Res., 33, 381-406, doi:10.1016/00777579(95)90054-3, 1995.

Brzezinski, M. A.: The Si : C:N ratio of marine diatoms: interspecific variability and the effect of some environmental variables, J. Phycol., 36, 87-96, doi:10.1111/j.0022-3646.1985.00347.x, 1985.

Burchard, H., Deleersnijder, E., and Meister, A.: A high-order conservative Patankar-type discretisation for stiff systems of production-destruction equations, Appl. Numer. Math., 47, 130, doi:10.1016/S0168-9274(03)00101-6, 2003.

Burchard, H., Bolding, K., Kühn, K., Meister, A., Neumann, T., and Umlauf, L.: Description of a flexible and extendable physicalbiogeochemical model system for the water column, J. Marine Syst., 61, 180-211, 2006.

Butenschön, M., Zavatarelli, M., and Vichi, M.: Sensitivity of a marine coupled physical biogeochemical model to time resolution, integration scheme and time splitting method, Ocean Model., 5253, 36-53, doi:10.1016/j.ocemod.2012.04.008, 2012.

Chesson, J.: The estimation and analysis of preference and its relatioship to foraging models, Ecology, 64, 1297-1304, doi:10.2307/1937838, 1983.

Chisholm, S.: Phytoplankton size, in: Primary Productivity and Biogeochemical Cycles in the Sea, edited by: Falkowski, P. and Woodhead, A., Plenum Press, New York, 213-237, 1992.

Chust, G., Allen, J. I., Bopp, L., Schrum, C., Holt, J., Tsiaras, K., Zavatarelli, M., Chifflet, M., Cannaby, H., Dadou, I., Daewel, U., Wakelin, S. L., Machu, E., Pushpadas, D., Butenschon, M., Artioli, Y., Petihakis, G., Smith, C., Garaçon, V., Goubanova, K., Le Vu, B., Fach, B. A., Salihoglu, B., Clementi, E., and Irigoien, X.: Biomass changes and trophic amplification of plankton in a warmer ocean, Global Change Biol., 20, 2124 2139, doi:10.1111/gcb.12562, 2014.

Daszykowski, M., Kaczmarek, K., Heyden, Y. V., and Walczak, B.: Robust statistics in data analysis - a review: basic concepts, Chemometr. Intell. Lab., 85, 203-219, doi:10.1016/j.chemolab.2006.06.016, 2007.
Dee, D. P., Uppala, S. M., Simmons, A. J., Berrisford, P., Poli, P., Kobayashi, S., Andrae, U., Balmaseda, M. A., Balsamo, G., Bauer, P., Bechtold, P., Beljaars, A. C. M., van de Berg, L., Bidlot, J., Bormann, N., Delsol, C., Dragani, R., Fuentes, M., Geer, A. J., Haimberger, L., Healy, S. B., Hersbach, H., Hólm, E. V., Isaksen, L., Kållberg, P., Köhler, M., Matricardi, M., McNally, A. P., Monge-Sanz, B. M., Morcrette, J.-J., Park, B.K., Peubey, C., de Rosnay, P., Tavolato, C., Thépaut, J.-N., and Vitart, F.: The ERA-Interim reanalysis: configuration and performance of the data assimilation system, Q. J. Roy. Meteorol. Soc., 137, 553-597, doi:10.1002/qj.828, 2011.

de Mora, L., Butenschön, M., and Allen, J. I.: How should sparse marine in situ measurements be compared to a continuous model: an example, Geosci. Model Dev., 6, 533-548, doi:10.5194/gmd6-533-2013, 2013.

Dickson, A. G.: Thermodynamics of the dissociation of boric acid in synthetic seawater from 273.15 to $318.15 \mathrm{~K}$, Deep-Sea Res., 37, 755-766, doi:10.1016/0198-0149(90)90004-F, 1990.

Dickson, A. G., Sabine, C. L., and Christian, J. R.: Guide to Best Practices for Ocean $\mathrm{CO}_{2}$ Measurements, PICES Special Publication 3, PICES, Sidney, British Columbia, available at: http://cdiac.ornl.gov/ftp/oceans/Handbook_2007/Guide_ all_in_one.pdf (last access: 14 August 2015), 2007.

Doney, S. C., Ruckelshaus, M., Emmett Duffy, J., Barry, J. P., Chan, F., English, C. A., Galindo, H. M., Grebmeier, J. M., Hollowed, A. B., Knowlton, N., Polovina, J., Rabalais, N. N., Sydeman, W. J., and Talley, L. D.: Climate change impacts on marine ecosystems, Annu. Rev. Mar. Sci., 4, 11-37, doi:10.1146/annurev-marine-041911-111611, 2012.

Droop, M. R.: The nutrient status of alga cells in continous culture, J. Mar. Biol. Assoc. UK, 54, 825-855, doi:10.1016/09247963(94)00031-6, 1974.

Dugdale, R. C., Wilkerson, F. P., and Minas, H. J.: The role of a silicate pump in driving new production, Deep-Sea Res. Pt. I, 42, 697-719, doi:10.1016/0967-0637(95)00015-X, 1995.

Ebenhöh, W., Kohlmeier, C., and Radford, P. J.: The benthic biological submodel in the European regional seas ecosystem model, Neth. J. Sea Res., 33, 423-452, doi:10.1016/00777579(95)90056-X, 1995.

Edwards, K. P., Barciela, R., and Butenschön, M.: Validation of the NEMO-ERSEM operational ecosystem model for the North West European Continental Shelf, Ocean Sci., 8, 983-1000, doi:10.5194/os-8-983-2012, 2012.

Falkowski, P. G. and Raven, J. A.: Aquatic photosynthesis, Princeton University Press, Princeton, USA, 2007.

Fasham, M. J. R., Ducklow, H. W., and McKelvie, S. M.: A nitrogen-based model of plankton dynamics in the oceanic mixed layer, J. Mar. Res., 48, 591-639, 1990.

Fennel, W.: A model of the yearly cycle of nutrients and plankton in the Baltic Sea, J. Marine Syst., 6, 313-329, doi:10.1016/09247963(94)00031-6, 1995.

Finkel, Z. V., Beardall, J., Flynn, K. J., Quigg, A., Rees, T. A. V., and Raven, J. A.: Phytoplankton in a changing world: cell size and elemental stoichiometry, J. Plankton Res., 32, 119-137, doi:10.1093/plankt/fbp098, 2010.

Flynn, K. J.: Ecological modelling in a sea of variable stoichiometry: dysfunctionality and the legacy of Redfield and Monod, Prog. Oceanogr., 84, 52-65, doi:10.1016/j.pocean.2009.09.006, 2010. 
Follows, M. J., Dutkiewicz, S., Grant, S., and Chisholm, S. W.: Emergent biogeography of microbial communities in a model ocean, Science, 315, 1843-1846, doi:10.1126/science.1138544, 2007.

Franks, P. J. S.: Planktonic ecosystem models: perplexing parameterizations and a failure to fail, J. Plankton Res., 31, 1299-1306, doi:10.1093/plankt/fbp069, 2009.

Gehlen, M., Gangst $\varnothing$, R., Schneider, B., Bopp, L., Aumont, O., and Ethe, C.: The fate of pelagic $\mathrm{CaCO}_{3}$ production in a high $\mathrm{CO}_{2}$ ocean: a model study, Biogeosciences, 4, 505-519, doi:10.5194/bg-4-505-2007, 2007.

Geider, R. J., MacIntyre, H. L., and Kana, T. M.: A dynamic model of phytoplankton growth and acclimation: responses of the balanced growth rate and chlorophyll $a$ : carbon ratio to light, nutrient limitation and temperature, Mar. Ecol-Prog. Ser., 148, 187200, doi:10.3354/meps148187, 1997.

Gentleman, W., Leising, A., Frost, B., Strom, S., and Murray, J.: Functional responses for zooplankton feeding on multiple resources: a review of assumptions and biological dynamics, DeepSea Res. Pt. II, 50, 2847-2875, doi:10.1016/j.dsr2.2003.07.001, 2003.

Gismervik, I.: Implications of zooplankton stoichiometry on distribution of $\mathrm{N}$ and $\mathrm{P}$ among planktonic size fractions, J. Plankton Res., 19, 343-356, doi:10.1093/plankt/19.3.343, 1997.

Glibert, P. M., Icarus Allen, J., Artioli, Y., Beusen, A., Bouwman, L., Harle, J., Holmes, R., and Holt, J.: Vulnerability of coastal ecosystems to changes in harmful algal bloom distribution in response to climate change: projections based on model analysis, Global Change Biol., 20, 3845-3858, doi:10.1111/gcb.12662, 2014.

Greenwood, N., Parker, E. R., Fernand, L., Sivyer, D. B., Weston, K., Painting, S. J., Kröger, S., Forster, R. M., Lees, H. E., Mills, D. K., and Laane, R. W. P. M.: Detection of low bottom water oxygen concentrations in the North Sea; implications for monitoring and assessment of ecosystem health, Biogeosciences, 7, 1357-1373, doi:10.5194/bg-7-1357-2010, 2010.

Hansell, D. A.: Recalcitrant dissolved organic carbon fractions, Annu. Rev. Mar. Sci., 5, 421-445, doi:10.1146/annurev-marine120710-100757, 2013.

Harris, R.: The L4 time-series: the first 20 years, J. Plankton Res., 32, 577-583, doi:10.1093/plankt/fbq021, 2010.

Heath, M., Roberston, W., Mardaljevic, J., and Gurney, W. S. G.: Modelling the population dynamics of Calanus in the Fair Isle current of northern Scotland, J. Sea Res., 38, 381-412, doi:10.1016/S1385-1101(97)00047-6, 1997.

Hirata, T., Hardman-Mountford, N. J., Brewin, R. J. W., Aiken, J., Barlow, R., Suzuki, K., Isada, T., Howell, E., Hashioka, T., Noguchi-Aita, M., and Yamanaka, Y.: Synoptic relationships between surface Chlorophyll- $a$ and diagnostic pigments specific to phytoplankton functional types, Biogeosciences, 8, 311-327, doi:10.5194/bg-8-311-2011, 2011.

Holt, J., Butenschön, M., Wakelin, S. L., Artioli, Y., and Allen, J. I.: Oceanic controls on the primary production of the northwest European continental shelf: model experiments under recent past conditions and a potential future scenario, Biogeosciences, 9, 97-117, doi:10.5194/bg-9-97-2012, 2012.

Holt, J. T. and James, I. D.: An s coordinate density evolving model of the northwest European continental shelf: 1. Model descrip- tion and density structure, J. Geophys. Res.-Oceans, 106, 1401514034, doi:10.1029/2000JC000304, 2001.

ICES: ICES Dataset on Ocean Hydrography, The International Council for the Exploration of the SeaCopenhagen, Copenhagen, Denmark, 2009.

Ingri, N., Kakolowicz, W., Sillén, L. G., and Warnqvist, B.: High-speed computers as a supplement to graphical methods V1: Haltafall, a general program for calculating the composition of equilibrium mixtures, Talanta, 14, 1261-1286, doi:10.1016/0039-9140(67)80203-0, 1967.

Jassby, A. D. and Platt, T.: Mathematical formulation of relationship between photosynthesis and light for phytoplankton, Limnol. Oceanogr., 21, 540-547, doi:10.4319/lo.1976.21.4.0540, 1976.

Jiao, N., Herndl, G. J., Hansell, D. A., Benner, R., Kattner, G., Wilhelm, S. W., Kirchman, D. L., Weinbauer, M. G., Luo, T., Chen, F., and Azam, F.: Microbial production of recalcitrant dissolved organic matter: long-term carbon storage in the global ocean, Nat. Rev. Microbiol., 8, 593-599, doi:10.1038/nrmicro2386, 2010.

Jiao, N., Robinson, C., Azam, F., Thomas, H., Baltar, F., Dang, H., Hardman-Mountford, N. J., Johnson, M., Kirchman, D. L., Koch, B. P., Legendre, L., Li, C., Liu, J., Luo, T., Luo, Y.-W., Mitra, A., Romanou, A., Tang, K., Wang, X., Zhang, C., and Zhang, R.: Mechanisms of microbial carbon sequestration in the ocean - future research directions, Biogeosciences, 11, 52855306, doi:10.5194/bg-11-5285-2014, 2014.

Jolliff, J. K., Kindle, J. C., Shulman, I., Penta, B., Friedrichs, M. A. M., Helber, R., and Arnone, R. A.: Summary diagrams for coupled hydrodynamic-ecosystem model skill assessment, J. Marine Syst., 76, 64-82, doi:10.1016/j.jmarsys.2008.05.014, 2009.

Keir, R. S.: The dissolution kinetics of biogenic calcium carbonates in seawater, Geochim. Cosmochim. Acta, 44, 241-252, doi:10.1016/0016-7037(80)90135-0, 1980.

Kleypas, J. A., Buddemeier, R., W., Archer, D., Gattuso, J.-P., Langdon, C., and Opdyke, B., N. : Geochemical consequences of increased atmospheric carbon dioxide on coral reefs, Science, 284, 118-120, doi:10.1126/science.284.5411.118, 1999.

Kohlmeier, C.: Modellierung des Spiekerooger Rückseitenwatts mit einem gekoppelten Euler-Lagrange-Modell auf der Basis von ERSEM, PhD thesis, Carl von Ossietzky Universität, Oldenburg, 2004.

Kwiatkowski, L., Yool, A., Allen, J. I., Anderson, T. R., Barciela, R., Buitenhuis, E. T., Butenschön, M., Enright, C., Halloran, P. R., Le Quéré, C., de Mora, L., Racault, M.-F., Sinha, B., Totterdell, I. J., and Cox, P. M.: iMarNet: an ocean biogeochemistry model intercomparison project within a common physical ocean modelling framework, Biogeosciences, 11, 7291-7304, doi:10.5194/bg-11-7291-2014, 2014.

Lee, K., Tong, L. T., Millero, F. J., Sabine, C. L., Dickson, A. G., Goyet, C., Park, G.-H., Wanninkhof, R., Feely, R. A., and Key, R. M.: Global relationships of total alkalinity with salinity and temperature in surface waters of the world's oceans, Geophys. Res. Lett., 33, L19605, doi:10.1029/2006GL027207, 2006.

Lee, Z., Du, K., and Arnone, R.: A model for the diffuse attenuation coefficient of downwelling irradiance, J. Geophys. Res., 110, 110, doi:10.1029/2004JC002275, 2005.

Leeuwen, S. M. v., Molen, J. v. d., Ruardij, P., Fernand, L., and Jickells, T.: Modelling the contribution of deep chlorophyll maxima 
to annual primary production in the North Sea, Biogeochemistry, 113, 137-152, doi:10.1007/s10533-012-9704-5, 2012.

Lenhart, H.-J., Mills, D. K., Baretta-Bekker, H., Leeuwen, S. M. v., Molen, J. v. d., Baretta, J. W., Blaas, M., Desmit, X., Kühn, W., Lacroix, G., Los, H. J., Ménesguen, A., Neves, R., Proctor, R., Ruardij, P., Skogen, M. D., Vanhoutte-Brunier, A., Villars, M. T., and Wakelin, S. L.: Predicting the consequences of nutrient reduction on the eutrophication status of the North Sea, J. Marine Syst., 81, 148-170, doi:10.1016/j.jmarsys.2009.12.014, 2010.

Litchman, E., Klausmeier, C. A., Schofield, O. M., and Falkowski, P. G.: The role of functional traits and trade-offs in structuring phytoplankton communities: scaling from cellular to ecosystem level, Ecol. Lett., 10, 1170-1181, doi:10.1111/j.14610248.2007.01117.x, 2007.

Litchman, E., Pinto, P. d. T., Klausmeier, C. A., Thomas, M. K., and Yoshiyama, K.: Linking traits to species diversity and community structure in phytoplankton, Hydrobiologia, 653, 15-28, doi:10.1007/s10750-010-0341-5, 2010.

Madec, G.: NEMO Ocean Engine, Note du Pole de modélisation, Institut Pierre-Simon Laplace (IPSL), No. 28, Paris, France, 2008.

Mehrbach, C., Culberson, C. H., Hawley, J. E., and Pytkowicx, R. M.: Measurement of the apparent dissociation constants of carbonic acid in seawater at atmospheric pressure, Limnol. Oceanogr., 18, 897-907, doi:10.4319/lo.1973.18.6.0897, 1973.

Millero, F. J.: Thermodynamics of the carbon dioxide system in the oceans, Geochim. Cosmochim. Acta, 59, 661-677, doi:10.1016/0016-7037(94)00354-O, 1995.

Millero, F. J., Lee, K., and Roche, M.: Distribution of alkalinity in the surface waters of the major oceans, Mar. Chem., 60, 111-130, doi:10.1016/S0304-4203(97)00084-4, 1998.

Moore, C. M., Mills, M. M., Arrigo, K. R., Berman-Frank, I., Bopp, L., Boyd, P. W., Galbraith, E. D., Geider, R. J., Guieu, C., Jaccard, S. L., Jickells, T. D., La Roche, J., Lenton, T. M., Mahowald, N. M., Maranon, E., Marinov, I., Moore, J. K., Nakatsuka, T., Oschlies, A., Saito, M. A., Thingstad, T. F., Tsuda, A., and Ulloa, O.: Processes and patterns of oceanic nutrient limitation, Nat. Geosci., 6, 701-710, doi:10.1038/ngeo1765, 2013.

Nightingale, P. D., Malin, G., Law, C. S., Watson, A. J., Liss, P. S., Liddicoat, M. I., Boutin, J., and Upstill-Goddard, R. C.: In situ evaluation of air-sea gas exchange parameterizations using novel conservative and volatile tracers, Global Biogeochem. Cy., 14, 373-387, doi:10.1029/1999GB900091, 2000.

Paasche, E.: Roles of nitrogen and phosphorus in coccolith formation in Emiliania huxleyi (Prymnesiophyceae), Eur. J. Phycol., 33, 33-42, 1998.

Patankar, S. V.: Numerical Heat Transfer and Fluid Flow, McGrawHill, New York, USA, 1980.

Platt, T., Harrison, W. G., Irwin, B., Horne, E. P., and Gallegos, C. L.: Photosynthesis and photoadaptation of marine phytoplankton in the arctic, Deep-Sea Res., 29, 1159-1170, doi:10.1016/0198-0149(82)90087-5, 1982.

Polimene, L., Allen, J. I., and Zavatarelli, M.: Model of interactions between dissolved organic carbon and bacteria in marine systems, Aquat. Microb. Ecol., 43, 127-138, doi:10.3354/ame043127, 2006.

Polimene, L., Pinardi, N., Zavatarelli, M., Allen, J. I., Giani, M., and Vichi, M.: A numerical simulation study of dissolved organic carbon accumulation in the northern Adriatic Sea, J. Geophys. Res.-Oceans, 112, C03S20, doi:10.1029/2006JC003529, 2007.

Polimene, L., Archer, S. D., Butenschön, M., and Allen, J. I.: A mechanistic explanation of the Sargasso Sea DMS "summer paradox", Biogeochemistry, 110, 243-255, doi:10.1007/s10533011-9674-z, 2012.

Polimene, L., Brunet, C., Butenschön, M., Martinez-Vicente, V., Widdicombe, C., Torres, R., and Allen, J. I.: Modelling a lightdriven phytoplankton succession, J. Plankton Res., 36, 214-229, doi:10.1093/plankt/fbt086, 2014.

Pujo-Pay, M. and Conan, P. and Raimbault, P.: Excretion of dissolved organic nitrogen by phytoplankton assessed by wet oxidation and ${ }^{15} \mathrm{~N}$ tracer procedures, Mar. Ecol.-Prog. Ser., 153, 99-111, doi:10.3354/meps153099, 1997.

Radford, P. and Joint, I.: The application of an ecosystem model to the Bristol Channel and Severn Estuary, Water Pollut. Control, 2, 244-250, 1980.

Ridgwell, A., Zondervan, I., Hargreaves, J. C., Bijma, J., and Lenton, T. M.: Assessing the potential long-term increase of oceanic fossil fuel $\mathrm{CO}_{2}$ uptake due to $\mathrm{CO}_{2}$-calcification feedback, Biogeosciences, 4, 481-492, doi:10.5194/bg-4-481-2007, 2007.

Riegman, R., Stolte, W., Noordeloos, A. A. M., and Slezak, D.: Nutrient uptake and alkaline phosphatase (ec $3: 1: 3: 1$ ) activity of emiliania huxleyi (PRYMNESIOPHYCEAE) during growth under $\mathrm{n}$ and $\mathrm{p}$ limitation in continuous cultures, J. Phycol., 36, 8796, doi:10.1046/j.1529-8817.2000.99023.x, 2000.

Ruardij, P. and Van Raaphorst, W.: Benthic nutrient regeneration in the ERSEM ecosystem model of the North Sea, Neth. J. Sea Res., 33, 453-483, doi:10.1016/0077-7579(95)90057-8, 1995.

Saux Picart, S., Butenschön, M., and Shutler, J. D.: Wavelet-based spatial comparison technique for analysing and evaluating twodimensional geophysical model fields, Geosci. Model Dev., 5, 223-230, doi:10.5194/gmd-5-223-2012, 2012.

Shutler, J. D., Smyth, T. J., Saux-Picart, S., Wakelin, S. L., Hyder, P., Orekhov, P., Grant, M. G., Tilstone, G. H., and Allen, J. I.: Evaluating the ability of a hydrodynamic ecosystem model to capture inter- and intra-annual spatial characteristics of chlorophyll$a$ in the north east Atlantic, J. Marine Syst., 88, 169-182, doi:10.1016/j.jmarsys.2011.03.013, 2011.

Smyth, T. J., Fishwick, J. R., Al-Moosawi, L., Cummings, D. G., Harris, C., Kitidis, V., Rees, A., Martinez-Vicente, V., and Woodward, E. M. S.: A broad spatio-temporal view of the Western English Channel observatory, J. Plankton Res., 32, 585-601, doi:10.1093/plankt/fbp128, 2010.

Steinberg, D. K., Carlson, C. A., Bates, N. R., Johnson, R. J., Michaels, A. F., and Knap, A. H.: Overview of the US JGOFS Bermuda Atlantic Time-series Study (BATS): a decade-scale look at ocean biology and biogeochemistry, Deep-Sea Res. Pt. II, 48, 1405-1447, doi:10.1016/S0967-0645(00)00148-X, 2001.

Stock, C. A., Dunne, J. P., and John, J. G.: Global-scale carbon and energy flows through the marine planktonic food web: an analysis with a coupled physical-biological model, Prog. Oceanogr. 120, 1-28, doi:10.1016/j.pocean.2013.07.001, 2014.

Sukenik, A., Bennett, J., and Falkowski, P.: Light-saturated photosynthesis Limitation by electron transport or carbon fixation?, BBA-Bioenergetics, 891, 205-215, doi:10.1016/00052728(87)90216-7, 1987. 
Takahashi, T., Williams, R. T., and Bos, D. L.: Carbonate chemistry, in: Chapter 3, GEOSECS Pacific Expedition, Hydrographic Data 1973-1974, Vol. 3, US Governemnt Priniting Office, Washington, D.C., USA, 1982.

Talmy, D., Blackford, J., Hardman-Mountford, N. J., Polimene, L., Follows, M. J., and Geider, R. J.: Flexible C : N ratio enhances metabolism of large phytoplankton when resource supply is intermittent, Biogeosciences, 11, 4881-4895, doi:10.5194/bg-114881-2014, 2014.

Timmermans, K. R., van der Wagt, B., Veldhuis, M. J. W., Maatman, A., and de Baar, H. J. W.: Physiological responses of three species of marine pico-phytoplankton to ammonium, phosphate, iron and light limitation, J. Sea Res., 53, 109-120, doi:10.1016/j.seares.2004.05.003, 2005.

van der Molen, J., Smith, H. C. M., Lepper, P., Limpenny, S., and Rees, J.: Predicting the large-scale consequences of offshore wind turbine array development on a North Sea ecosystem, Cont. Shelf Res., 85, 60-72, doi:10.1016/j.csr.2014.05.018, 2014.

van Duyl, F. C., van Raaphorst, W., and Kop, A. J.: Benthic bacterial production and nutrient sediment-water exchange in sandy North-Sea sediments, Mar. Ecol-Prog. Ser., 100, 85-95, doi:10.3354/meps 100085, 1993.

Varela, R. A., Cruzado, A., and Gabaldón, J. E.: Modelling primary production in the North Sea using the European Regional Seas Ecosystem Model, Neth. J. Sea Res., 33, 337-361, doi:10.1016/0077-7579(95)90052-7, 1995.

Veldhuis, M. J. W., Timmermans, K. R., Croot, P., and van der Wagt, B.: Picophytoplankton; a comparative study of their biochemical composition and photosynthetic properties, J. Sea Res., 53, 7-24, doi:10.1016/j.seares.2004.01.006, 2005.

Vichi, M., Ruardij, P., and Baretta, J. W.: Link or sink: a modelling interpretation of the open Baltic biogeochemistry, Biogeosciences, 1, 79-100, doi:10.5194/bg-1-79-2004, 2004.

Vichi, M., Pinardi, N., and Masina, S.: A generalized model of pelagic biogeochemistry for the global ocean ecosystem. Part I: Theory, J. Marine Syst., 64, 89-109, 2007.

Villarino, E., Chust, G., Licandro, P., Butenschön, M., Ibaibarriaga, L., Larrañaga, A., and Irigoien, X.: Modelling the future biogeography of North Atlantic zooplankton communities in response to climate change, Mar. Ecol.-Prog. Ser., 531, 121-142, doi:10.3354/meps11299, 2015.
Wakelin, S. L., Holt, J. T., Blackford, J. C., Allen, J. I., Butenschön, M., and Artioli, Y.: Modeling the carbon fluxes of the northwest European continental shelf: validation and budgets, J. Geophys. Res., 117, C05020, doi:10.1029/2011JC007402, 2012.

Walve, J. and Larsson, U.: Carbon, nitrogen and phosphorus stoichiometry of crustacean zooplankton in the Baltic Sea: implications for nutrient recycling, J. Plankton Res., 21, 2309-2321, doi:10.1093/plankt/21.12.2309, 1999

Ward, B. B.: Chapter 5 - Nitrification in marine systems, in: Nitrogen in the marine environment, 2nd Edn., edited by: Capone, D. G., Bronk, D. A., Mulholland, M. R., and Carpenter, E. J., Plenum Press, New York, 199-261, 1992.

Weiss, R. F.: The solubility of nitrogen, oxygen and argon in water and seawater, Deep-Sea Res., 17, 721-735, doi:10.1016/00117471(70)90037-9, 1970.

Weiss, R. F.: Carbon dioxide in water and seawater: the solubility of a non-ideal gas, Mar. Chem., 2, 203-215, doi:10.1016/03044203(74)90015-2, 1974.

Weston, K., Fernand, L., Nicholls, J., Marca-Bell, A., Mills, D., Sivyer, D., and Trimmer, M.: Sedimentary and water column processes in the Oyster Grounds: A potentially hypoxic region of the North Sea, Mar. Environ. Res., 65, 235-249, doi:10.1016/j.marenvres.2007.11.002, 2008.

Wild-Allen, K., Herzfeld, M., Thompson, P. A., Rosebrock, U., Parslow, J., and Volkman, J. K.: Applied coastal biogeochemical modelling to quantify the environmental impact of fish farm nutrients and inform managers, J. Marine Syst., 81, 134-147, doi:10.1016/j.jmarsys.2009.12.013, 2010.

Yool, A., Popova, E. E., and Anderson, T. R.: MEDUSA-2.0: an intermediate complexity biogeochemical model of the marine carbon cycle for climate change and ocean acidification studies, Geosci. Model Dev., 6, 1767-1811, doi:10.5194/gmd-6-17672013, 2013.

Zavatarelli, M. and Pinardi, N.: The Adriatic Sea modelling system: a nested approach, Ann. Geophys., 21, 345-364, doi:10.5194/angeo-21-345-2003, 2003.

Zeebe, R. W. and Wolf-Gladrow, D.: $\mathrm{CO}_{2}$ in Seawater: Equilibrium, Kinetics, Isotopes, no. 65 in Elsevier Oceanography Series, Elsevier, Amsterdam, the Netherlands, 2001. 